\author{
Universidade de São Paulo \\ Instituto de Física
}

\title{
Espectroscopia de correlação de ruído em Transparência Eletromagneticamente Induzida em átomos frios
}

\author{
Hans Marín Florez \\ Dissertação de Mestrado apresentada ao \\ Instituto de Física para a obtenção \\ do título de Mestre em Ciências.
}

Orientador:

Prof. Dr. Marcelo Martinelli

Comissão Examinadora:

Prof. Dr. Marcelo Martinelli - IFUSP

Prof. Dr. Antônio F.R. de Toledo Piza - IFUSP

Prof. Dr. Reinaldo L. Cavasso - UNICAMP

São Paulo

2012 


\section{Resumo}

Por meio da espectroscopia de ruído, estudamos a correlação entre dois feixes de luz com polarizações circulares opostas, acoplando a transição ${ }^{5} S_{1 / 2} F=2$ à ${ }^{5} P_{3 / 2} F^{\prime}=2$ em configuração $\Lambda$ de Transparência Eletromagneticamente Induzida (EIT sigla em inglês) com átomos frios de Rubídio 85. Observamos a mudança de correlação para anti-correlação entre os feixes de prova e controle fora da ressonância, conforme aumentamos a sua potência. Esta transição é uma consequência da competição que existe entre a absorção de um fóton no sistema $\Lambda$, responsável pelo regime de correlação, e as transições Stokes e Anti-Stokes que produzem a anti-correlação dos feixes.

A partir dos espectros do sinal médio da intensidade e da correlação de ruído observamos, nesta última, que o pico de EIT é livre de alargamento por potência, o que possibilita que a medida do tempo de coerência entre os níveis fundamentais na condição de EIT seja mais precisa. Usando o modelo de Difusão de Fase para campos em EIT em configuração $\Lambda$, observamos que os espectros teóricos de correlação estão de acordo com os dados experimentais. Isto mostra que esta teoria modela um sistema que envolve níveis hipefinos com degenerescência, através de um sistema simples de 3 níveis. O modelo também prevê o não-alargamento do pico de correlação

e mostramos ser esta uma medida mais fina de caracterização da largura de linha em comparação ao sinal médio da intensidade. 


\begin{abstract}
Using noise espectroscopy, we have studied the correlation between two laser beams with opposite circular polarizations, coupling the transition ${ }^{5} S_{1 / 2} F=2$ to ${ }^{5} P_{3 / 2} F^{\prime}=2$ in $\Lambda$-type Electromagnetically Induzed Transparency (EIT), using cold atoms of ${ }^{85} \mathrm{Rb}$. We have observed the transition from correlation to anti-correlation, between the slightly off-resonance probe and control beams, when the intensity of both beams was increased. This transition is a consequence of the competition between the single photon transition in a $\Lambda$ system, which is responsible for the correlation regime, and the Stoke and Anti-Stoke transitions producing anti-correlation between both beams.

Studying the spectra of the intensity mean value and the noise correlation, we observed in the last one that the EIT peak is free of power broadening, which makes it possible to preciselly measure the coherence time between the ground states in EIT condition. Using the Phase Difusion model for the lasers in a $\Lambda$ EIT system, we show that the theoretical correlation spectra are in good accordance with the experimental data. This theory models a system with degenerate hyperfine levels, using a simple three-level model. Moreover, the model predicts the non-broadening of the correlation peak, showing the correlation as a more accurated measurement than the intensity mean value.
\end{abstract}




\section{Agradecimentos}

Agradeço primeramente ao Prof. Marcelo Martinelli pelo apoio e orientação durante este trabalho. Com ele tive a possibilidade de entrar no mundo da física experimental, trazendo muitos dos conceitos teóricos para a realidade do laboratório. Sou grato também por sua ajuda para me adaptar na chegada ao Brasil.

Ao Prof. Paulo Nussenzveig, com quem apredi as sutilezas dos conceitos da física fundamental e física quântica. Admiro sua empolgação ao discutir física. nas discussões de física.

À minha familia: meu pai e minha mãe, por nunca desistirem de me mostrar as responsabilidades que adquiriram no transcurso da vida e como os sacrifícios tem suas recompensas. Eles sempre se preocuparam com minha educação, tanto na escola quanto na educação superior. Gracias a ellos logre llegar hasta aqui. Ao meu irmão que me encaminhou ao mundo da física e me deu grandes conselhos. Com a sua experiência profissional como exemplo, me motivei para traçar o meu próprio caminho.

Ao Antonio, "Macaxeira", e ao Felippe, "Phenomenon", grandes amigos e físicos. Eles fizeram minha chegada ao Brasil mais amena, me ajudando a procurar moradia e me adaptar com o sistema da universidade. Agradeço a eles também, pelas interesantes discussões de física e pelas histórias da vida profissional com as quais aprendi bastante. Não posso esquecer dos momentos agradáveis durante o trabalho no laboratório e fora dele.

Agradeço ao Laércio, que me ensinou grande parte do funcionamento do experimento, durante o pouco tempo que tivemos contato. Ao Helio que começou o processo de construção da armadilha magneto-ótica e o controle dos átomos frios. Ao Rodrigo que contribuiu na caracterização dos átomos frios mediante espectroscopia Raman. Ao Carlos Garrido, José Aguirre, Paulo Valente, Daniel Felinto pelos trabalhos feitos sobre EIT e análise de flutuações dos campos eletromagnéticos em condição de EIT. Ao Arturo Lezama pelos trabalhos de análise de ruído em EIT para sistemas de dois níveis, em colaboração com nosso grupo.

Aos meus colegas que posteriomente chegaram ao laboratório, a Paula, a Klara, a Rayssa, o Igor, o Luiz e o Carlos pelas discussões sobre física e pelos bons momentos durante o café no laboratório. Ao Márcio que durante o trabalho conjunto 
aprendi ainda mais sobre pesquisa por conta de sua experiência adquirida em outros laboratórios e por ter tornado o ambiente de trabalho bastante divertido.

Ao Prof. Luciano Cruz , pelas discussões sobre física e pelo aprendizado na área de Física atômica, aproveitando cada momento para trocar experiências na "luta" com o experimento. Ao Prof. Daniel Felinto com que tive a oportunidade de trabalhar diretamente durante um mês no Laboratório de Átomos frios na UFPE. Agradeço pelo que aprendi sobre física atômica e técnicas experimentais.

Agradeço à Eliane e ao Tiago, pela convivência durante quase um ano e meio, tendo que suportar a minha desordem durante este tempo.

Um agradecimento especial ao meus amigos Colombianos, Brasileiros e Mexicanos, que fiz durante o Mestrado e com os quais aproveitei tantos momentos de troca cultural durante minha estadia aqui no Brasil, contribuindo assim, enormemente para minha formação pessoal. Não quero mencionar em detalhe cada um deles, para não ter o risco de esquecer alguém.

Quero agradecer de modo especial, à Ana, que fez meus últimos seis meses no Brasil, se tornarem uma experiência única de vida, e espero continue sendo uma motivação constante no meu trabalho. Aliás, sem ela, esta dissertação teria sido escrita numa outra língua que exgiria do leitor, um conhecimento básico de "portunhol". Gracias a ti, y gracias a todos.

Agradeço a Maria Izabel, Eber e Cristiano, da secretaria da CPG, pela sua ajuda com os muitos processos burocráticos, de tantos precisei passar. Igualmente a Juliane a Edi, secretárias da FEP, que também me ajudaram neste tipo de situações.

Por fim, agradeço a CNPq, por seu apoio financiero durante este trabalho. 

1 O que é Transparência Eletromagneticamente Induzida? 1

1.1 Efeitos das interações entre átomos e luz . . . . . . . . . . . . . . . . . . 2

1.2 Sistema átomo-campo . . . . . . . . . . . . . . . . 2

1.3 Estado escuro $\left|\psi_{D}\right\rangle \ldots \ldots \ldots \ldots$. . . . . . . . . . . . 4

1.4 EIT em sistemas de 3 níveis . . . . . . . . . . . . . . . . 8

1.5 EIT em sistemas de 2 níveis com degenerescências . . . . . . . . . . . 15

1.5.1 Interação com campo magnético . . . . . . . . . . . . . . . 19

1.5.2 Contribuições dissipativas . . . . . . . . . . . . . . . . 21

1.6 Espaço de Liouville . . . . . . . . . . . . . . . . . . . 22

2 Modelo de Difusão de fase para o ruído e a correlação dos campos eletromágneticos, em sistema de 3 níveis $\quad 25$

2.1 Matriz de Covariância . . . . . . . . . . . . . . . 31

2.2 Espectro de ruído dos campos de prova e de controle . . . . . . . . 33

3 Preparação e controle experimental dos átomos frios 35

3.1 Estrutura hiperfina dos átomos de Rubídio . . . . . . . . . . . . . 35

3.2 Resfriamento e aprisionamento de átomos neutros . . . . . . . . . . . 37

3.3 Temperatura e número de átomos aprisonados . . . . . . . . . . . . . 40

3.4 Desligamento do campo magnético de aprisonamento . . . . . . . . . 43

3.5 Esquema de deteção e aquisição de dados . . . . . . . . . . . . . . . . 46 
4 Espectroscopia de absorção em configuração de EIT de átomos frios de Rubídio 85

4.1 Montagem Experimental . . . . . . . . . . . . . . . . 54

4.2 Espectroscopia de absorção da nuvem atômica do estado fundamental $\mathrm{F}=2$ do $\mathrm{Rb}^{85} \ldots \ldots \ldots \ldots \ldots \ldots \ldots$

4.3 EIT na transição de $F=2$ para $F^{\prime}=2$ com átomos frios de $\mathrm{Rb}^{85}$. . 59

4.4 Espectroscopia de ruído e correlação na configuração de EIT com átomos frios . . . . . . . . . . . . . . . . . . 61

4.5 Espectroscopia de absorção, de ruído e da correlação na condição de Hanle-EIT . . . . . . . . . . . . . . . . . . . . . 71

$\begin{array}{llr}5 & \text { Conclusões e perspetivas } & 77\end{array}$

A Software de controle e aquisição $\quad 81$

A.1 Programa de Controle da MOT . . . . . . . . . . . . . . . . . . . . . 81

A.2 Programa de Aquisição . . . . . . . . . . . . . . . . . . . . 85

$\begin{array}{ll}\text { Bibliografia } & 93\end{array}$ 


\section{Introdução}

Recentemente, a ótica quântica e a informação quântica desenvolveram-se estudando a natureza quântica da luz e o processamento de informação em sistemas quânticos. Isto permitiu estudar a manipulação de átomos mediante a interação com a luz, e a análise da informação que pode estar contida nela, observando fenômenos como superposição e emaranhamento. Este último abriu toda uma discussão sobre os fundamentos da mecânica quântica, como no "Paradoxo de EPR", inicialmente abordado por Einstein et al. em 1935 [1] o que posteriormente motivou uma prova experimental em [2].

No desenvolvimento da ótica quântica e da informação quântica, o estudo da manipulação coerente de átomos com luz tem um papel importante para estudar protocolos de processamento e armazenamento de informação. No caso de transporte de informação, a luz é um excelente candidato, com o qual pode-se estudar emaranhamento e teletransporte quântico. Por outro lado os átomos possuem características que permitem usa-los em protocolos de procesamento e armazenamento de informação [3].

Uma aplicação da manipulação coerente de átomos é o resfriamento e aprisionamento, em armadilhas magneto-óticas (MOT-Magneto Optical Trap) [4, 5, 6, 7], armadilhas óticas de dipolo [8], chegando até condensados de Bose-Einstein [9] manipulando espacialmente os átomos. Um outro fenômeno que envolve a manipulação coerente de átomos com luz é a Transparência Eletromagneticamente Induzida (EIT sigla em inglês). Este efeito ocorre quando o bombeamento ótico entre um campo de prova e um campo de controle, acoplados a um sistema de 3 níveis, entra em interferência destrutiva. Esta interferência entre canais de excitação faz com que no ponto de ressonância Raman (em que obtemos a maxima absorção para um sistema simples de 2 níveis), obtemos transparência do meio atômico [10]. Este efeito foi visto da primeira vez em [11] por Boller et al em átomos quentes. Posteriormente foi observando em átomos frios $[12,13]$. Neste caso, manipulamos os graus de liberdade internos dos átomos. 
Na ótica quântica, no dominio das variáveis contínuas, podemos estudar as flutuações dos campos eletromagnéticos, em que temos informação sobre a estatística dos campos, como os campos térmicos, campos de Fock, campos coerentes e campos comprimidos ou squeezed. Os campos de squeezing se caracterizam por ter uma diminuição de ruído em alguma das suas quadraturas (amplitude ou fase), em relação com o ruído limite determinado pelo Standart Quantum Limit [14]. A compressão de ruído foi vista inicialmente por Kimble em [15], usando o Oscilador Paramétrico Ótico (OPO). Na interação de átomos como luz coerente, mediante processos óticos não lineares, como a mistura se quatro ondas (4WM), observou-se a compressão de ruído das quadraturas de fase e amplitude [16, 17]. Por outro lado, o ruído dos campos eletromagnéticos fazem parte dos critérios de emaranhamento no contexto de varíaveis contínuas, entre esses o critério de Duan et al [18]. Este critério foi implementado no experimento de 4WM [19], mostrando que os campos de prova e seu conjugado possuíam um alto grau de correlação, de modo que eles eram campos emaranhados.

Uma das características do fenômeno de EIT é a diminuição da velocidade de grupo dos campos eletromagnéticos, de modo que a ela é muito menor que no caso de dispersão normal em sistemas de dois níveis [20, 21]. Por outro lado, o efeito de EIT é usado para obter o efeito de 4WM, como foi demostrado por Braje et al. em [22] usando átomos frios. Estes dois efeitos são usados em protocolos de armazenamento de luz no meio atômico [23]. Ao estudar as propriedades da luz durante o seu armazenamento, temos o efeito de memória quântica, em que armazenamos estados quânticos da luz em coerências atômicas [21, 24, 25, 26, 27], o que leva à correlação entre os campos que escrevem a informação na memória.

O estudo das flutuações de campos eletromagneticos em condição de EIT já feito no Laboratório de Manipulação Coerente de Átomos e Luz (LMCAL) em células de vapor $[28,29,30]$. Em [31, 32] foi reportada a correlação e anti-correlção do ruído dos campos em configuração de EIT, para átomos em células de vapor. Posteriormente, no LMCAL com L. Cruz et al em [33], reportou-se este mesmo efeito, analisando o ruído no espeço de frequências, mediante o modelo de Difusão de Fase. Nesse trabalho é feito um estudo quantitativo da transição de correlação para anti-correlação, conforme aumentamos a intensidade dos campos. Em [34], é feita uma espectroscopia da correlação dos campos em condição de EIT, para átomos em células de vapor. Como conclusão, reportou-se a correlação como um parâmetro livre de alargamento por potência. No entanto, não se faz uma clara discussão entre a dependência por potência do espectro de absorção em relação ao não alargamento do espectro da correlação. Este efeito poderia ter aplicação na linha de metrologia, em que o pico do espectro da correlação seria um parâmetro mais fino para caracterizar a taxa intrinseca de coerência entre os níveis fundamentais induzida em EIT. 
Neste trabalho, estudaremos a absorção dos campos eletromagnéticos em condição de EIT e Hanle-EIT, junto com as flutuações e a correlação entre os campos, para átomos frios de Rubídio. Mostraremos como a correlação induzida entre os campos pode se transformar em anti-correlação, conforme aumentamos a potência deles. Analisaremos as diferenças entre os espectros de absorção e correlação, em que este último, apresenta características mais finas à medida que aumentamos as intensidades dos campos. Mostraremos que estes resultados experimentais podem ser muito bem descritos pelo modelo de Difusão de Fase.

No primeiro capítulo deste trabalho apresentaremos um estudo teórico sobre o fenômeno de EIT em sistemas de 3 níveis, passando pelo sistema de 2 níveis com degenerescências em presença de campos magnéticos, para estudar o efeito de HanleEIT. No capítulo 2 descreveremos o modelo de Difusão de Fase, que é usado para estudar e descrever, o ruído dos campos eletromagneticos em condição de EIT e Hanle-EIT. No capítulo 3 mostraremos a preparação experimental necessária para fazer a espectroscopia da nuvem atômica. Isto é, o resfriamento e aprisionamento de átomos caracterizado pela temperatura e pelo número de átomos da nuvem, o desligamento de campos magnéticos necessário para não modificar as estruturas de níveis atômicos, e o esquema de deteção adaptado para medir o sinal DC e o sinal de alta frequência da luz. Finalmente, no capítulo 4, mostraremos os resultados das medições de espectroscopia da nuvem em condição de EIT e Hanle-EIT. 


\section{CAPÍTULO 1}

\section{O que é Transparência Eletromagneticamente Induzida?}

Neste capítulo começamos apresentando os conceitos teóricos que permitem abordar o fenômeno de Transperência Eletromagneticamente Induzida (EIT - Electromagnetically Induced Transparency), como base para o entendimento e análise das experiências feitas no laboratório, cujos resultados vão ser discutidos no capítulo 4. Analisaremos as condições necessárias para que a transparência induzida aconteça em sistemas de três níveis, que é o sistema mais simples para observa-la [10, 11, 12, 35]. Posteriormente analisaremos a transparência em sistemas de dois níveis degenerados, dentro do qual estudaremos o fenômeno de Hanle-EIT, que envolve absorção de luz com polarizações específicas devida à quebra da degenerescência mediante campos magnéticos [28, 36, 37].

Adicionalmente, sendo uma das principais ferramentas em ótica quântica, intruduziremos o conceito de ruído dos campos electromagnéticos, relacionados ao ruído da suas quadraturas. Torna-se interesante estudar o ruído dos campos eletromagnéticos quando interagem com o meio atômico em condição de EIT, porque este fenômeno pode induzir a correlação de ruído entre os dois campos distintos, mediante uma tranformação de ruído de fase para ruído de amplitude devido ao meio atômico, entre outros efeitos.

Na primeira parte do capítulo, definiremos a estrutura de níveis do sistema atômico que estudaremos. Abordaremos o fenômeno de aprisionamento coerente de população (CPT - Coherent Population Trapping). Na segunda parte, será feita a descrição de EIT para sistemas atômicos de 3 níveis e 2 níveis degenerados, junto com a descrição para Hanle-EIT. 


\subsection{Efeitos das interações entre átomos e luz}

Para entender um pouco o contexto do fenômeno de EIT, vamos considerar o caso mais simples em que um campo eletromagnético de prova $\vec{E}_{p}(t)$ é acoplado a um átomo de dois níveis de energia (trata-se de um caso simples de analisar, mas na realidade lidamos com átomos de múltiplos níveis). Durante a interação o átomo adquire uma polarização que é proporcional ao campo de prova incidente, ou seja $\vec{P}_{\text {atm }}=\epsilon_{o} \chi^{(1)} \vec{E}_{p}$, onde $\epsilon_{o}$ é a permissividade elétrica no vácuo e $\chi^{(1)}$ é a susceptibilidade linear elétrica. Neste processo o átomo absorve a luz e num tempo caraterístico depois (tempo de decaimento) ele emite e espalha luz. As informações do que acontece com a absorção e dispersão da luz do campo de prova estão contidas na parte real e imaginária da polarização $\vec{P}_{\text {atm }}$, respectivamente. A absorção do meio atômico é representada mediante um perfil lorentziano em função da frequência do campo de prova incidente, como é mostrado na figura (1.1.a). O máximo de absorção acontece quando a frequência $\omega_{1}$ do campo de prova corresponde à diferença de frequência entre os níveis de energia acoplados.

Agora, consideremos o sistema mais real em que os átomos possuem vários níveis atômicos, de modo que além do campo de prova $\vec{E}_{p}$ acoplando uma transição dada, temos também um campo de controle $\vec{E}_{c}$ acoplando o mesmo estado excitado, porém com um estado fundamental diferente. Esta configuração de acoplamento, entre os dois campos e um átomo de três níveis, é conhecida como configuração tipo $\Lambda^{1}$ mostrada na figura (1.1.b). Quando este campo adicional é acoplado com o meio atômico, de imediato pensa-se que este vai afetar a absorção e dispersão do campo de prova, dentro de algumas condições específicas. Se a dessintonia de cada um dos campos é muito próxima uma da outra, os possíveis canais de transição podem entrar em inteferência construtiva ou destrutiva [35]. Justamente, quando a dessintonia dos campos satisfaz a condição $\operatorname{Raman}\left(\delta=\Delta_{1}-\Delta_{2}=0\right)$, o sistema pode apresentar interferência destrutiva na absorção para uma dada fase entre o bombeio dos dois campos. Isso leva consequentemente ao cancelamento de absorção na transmissão da luz, quando o campo está em ressonância com a transição atômica. Adicionalmente, na condição de EIT, conseguimos obter uma propagação lenta da luz, que pode ser aplicada no armazenamento de luz em átomos [23, 21].

\subsection{Sistema átomo-campo}

Consideremos um sistema atômico de 3 níveis em configuração $\Lambda$ como é mostrado na figura (1.1.b), em que o campo $\vec{E}_{p}$ com frequência $\omega_{p}$ acopla a transição entre o

\footnotetext{
${ }^{1}$ Existem varios tipos de configurações para acoplar sistemas de três níveis em condição de EIT. Temos configuração tipo $\Lambda, V$ e em cascata. Pode-se encontrar mais sobre esse tipos de configuração em [35]
} 


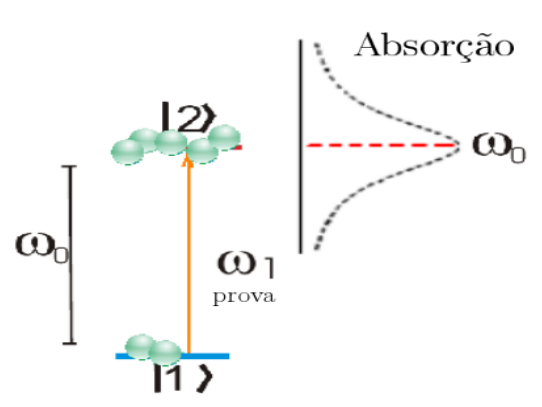

( a )

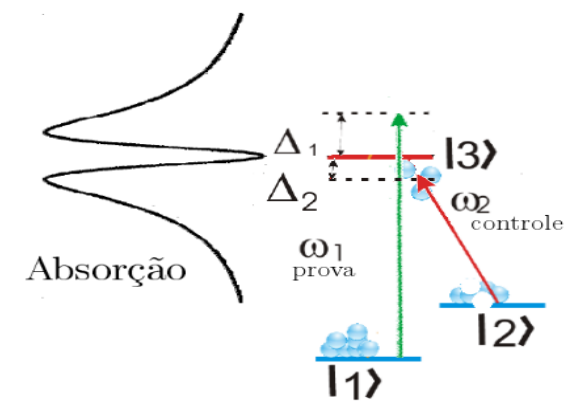

(b)

Figura 1.1: Espectro de absorção do campo electromagnéticos acoplado com (a) Sistema de dois níveis com polarização linear. (b) Sistema de três níveis.

estado $|1\rangle$ e o estado $|3\rangle$, e o campo campo $\vec{E}_{c}$ com frequência $\omega_{c}$ acopla a transição entre os estados $|2\rangle$ e $|3\rangle$. Os estados $|1\rangle,|2\rangle$ e $|3\rangle$ são auto-estados do hamiltoniano, que descreve unicamente o sistema atômico livre, formando uma base ortogonal de estados. Mediante uma descrição semiclássica do sistema átomo-campo, na qual os observáveis atômicos são quantizados enquanto as variáveis do campo são consideradas clássicas, o hamiltoniano do sistema é dado por

$$
H=H_{o}+H_{\text {int }},
$$

onde $H_{o}$ é o hamiltoniano dos átomos em condição livre e $H_{\text {int }}$ é o hamiltoniano de interação dos átomos com os campos eletromagnéticos. O hamiltoniano responsável pela evolução temporal livre dos átomos é definido como

$$
H_{o}=\sum_{j=1}^{3} \hbar \omega_{j} \hat{\sigma}_{j} ; \quad \hat{\sigma}_{j}=|j\rangle\langle j|
$$

onde $\omega_{j}$ é a frequência de transição de cada nível atômico em relação ao nível de energia de ionização e $\sigma_{j}$ corresponde à população atômica de cada nível. O hamiltoniano de interação $H_{\text {int }}$ é dado por

$$
H_{\mathrm{int}}=\hbar\left[\Omega_{p} \hat{\sigma}_{13} e^{i\left(\omega_{p} t+\phi_{p}\right)}+\Omega_{c} \hat{\sigma}_{23} e^{i\left(\omega_{c} t+\phi_{p}\right)}+H . c .\right],
$$

onde $\Omega_{p}=-\vec{d}_{13} \cdot \vec{E}_{o p} / \hbar$ e $\Omega_{c}=-\vec{d}_{23} \cdot \vec{E}_{o c} / \hbar$ corresponde às frequências de Rabi do campo de prova e o campo de controle, respectivamente, sendo $\vec{d}_{i j}$ o dipolo elétrico para a transição entre os níveis $i$ e $j$. Cada um dos campos possui uma frequência $\omega_{p}$ e $\omega_{c}$, que em geral tem uma dessintonia com respeito à frequência de transição $\omega_{13}$ e $\omega_{23}$ entre estados fundamendais $|1\rangle$ e $|2\rangle$, e o estado excitado $|3\rangle$. Finalmente consideramos as fases dos campos $\phi_{p}$ e $\phi_{c}$, que terão um papel muito importante 
no modelo de difusão de fase descrito no capítulo 2, para calcular os ruídos e a correlação dos dois campos.

\subsection{Estado escuro $\left|\psi_{D}\right\rangle$}

Para explicar detalhadamente o fenômeno de EIT, começaremos analisando os estados atômicos envolvidos neste fenômeno e a dinâmica do sistema para chegar na condição de transparência induzida. Mediante o formalismo de interação, em que o estado do sistema é descrito pelo estado $\left|\psi_{I}(t)\right\rangle=e^{\frac{i H_{o} t}{\hbar}}|\psi(t)\rangle$, o hamiltoniano de interação se transforma em

$$
H_{\mathrm{int}}^{\prime}=e^{\frac{i H_{o} t}{\hbar}} H_{\mathrm{int}} e^{-\frac{i H_{o} t}{\hbar}}=\hbar\left[\Omega_{p} \hat{\sigma}_{13} e^{i\left(\Delta_{1} t-\phi_{p}\right)}+\Omega_{c} \hat{\sigma}_{23} e^{i\left(\Delta_{2} t-\phi_{c}\right)}+H . c .\right]
$$

onde $\Delta_{1}$ e $\Delta_{2}$ são as dessintonias do campo de prova e acomplamento, respectivamente. No entanto, podemos analisar o caso quando os campos estão em ressonância com as transições atômicas, ou seja $\Delta_{1}=\Delta_{2}=0$, em que a representação matricial do hamiltoniano de interação é

$$
H_{\mathrm{int}}^{\prime}=\hbar\left(\begin{array}{ccc}
0 & 0 & \Omega_{p} e^{-i \phi_{p}} \\
0 & 0 & \Omega_{c} e^{-i \phi_{c}} \\
\Omega_{p} e^{i \phi_{p}} & \Omega_{c} e^{i \phi_{c}} & 0
\end{array}\right)
$$

A partir desta condição de ressonância é possível determinar os auto-estados do hamiltoniano da eq. 1.1, que vão ter um papel determinante na transperência dos campos. Os auto-estados do hamiltoniano de interação, na base de estados $\{|1\rangle,|2\rangle,|3\rangle\}$ do hamiltoniano livre $H_{o}$, são

- Estado Escuro com autovalor $\lambda_{D}=0$

$$
\left|\psi_{D}\right\rangle=\cos \theta e^{-i \phi_{p}}|1\rangle-\sin \theta e^{-i \phi_{c}}|2\rangle
$$

onde $\tan \theta=\Omega_{c} / \Omega_{p}$. Este estado mostra que a probabilidade de achar o sistema no estado exitado $|3\rangle$ é nulo.

- Estados $|\psi \pm\rangle$ com autovalores $\lambda_{ \pm}= \pm \hbar \Omega$ sendo $\Omega=\sqrt{\left|\Omega_{c}\right|^{2}+\left|\Omega_{p}\right|^{2}}$

$$
\left|\psi_{ \pm}\right\rangle=\frac{1}{\sqrt{2}}\left(|3\rangle \pm\left|\psi_{A}\right\rangle\right)
$$

onde $\left|\psi_{A}\right\rangle=\sin \theta e^{i \phi_{p}}|1\rangle+\cos \theta e^{i \phi_{c}}|2\rangle$ definido como o estado de acoplamento. 
Agora, é importante analisar a dinâmica dos átomos para chegar num desses autoestados. Estão os átomos preparados no estado escuro do começo? Como é possível chegar no estado escuro? Para responder a essas perguntas, devemos estudar a evolução temporal do nosso sistema. Incialmente faremos uma abordagem em que o sistema é fechado, ou seja, não tem nenhum tipo de perda de energia, e consideraremos a evolução temporal definida por

$$
i \hbar \frac{d\left|\psi_{I}(t)\right\rangle}{d t}=H_{\mathrm{int}}^{\prime}\left|\psi_{I}(t)\right\rangle \text {. }
$$

Devido ao fato do hamiltoniano de interação $H_{\text {int }}^{\prime}$ ser independente do tempo, o estado do sistema para um tempo $t$ é dado por

$$
\left|\psi_{I}(t)\right\rangle=e^{\frac{i H_{\text {int }}^{\prime}}{\hbar}}\left|\psi_{I}(0)\right\rangle .
$$

Para ver de forma clara o estado escuro como um efeito de interferência, vamos considerar o estado inicial do sistema como uma superposição arbitrária entre os estados fundamentais, como é feito em [30], ou seja

$$
\left|\psi_{I}(0)\right\rangle=c_{1}|1\rangle+c_{2}|2\rangle ; \quad\left|c_{1}\right|^{2}+\left|c_{2}\right|^{2}=1 \text {. }
$$

Invertemos as equações (1.6) e (1.7), tal que os estados $|1\rangle,|2\rangle$ e $|3\rangle$, são expressados em termos dos auto-estados do hamiltoniano de interação $\left|\psi_{D}\right\rangle$ e $\left|\psi_{A}\right\rangle=$ $\left(\left|\psi_{+}\right\rangle-\left|\psi_{-}\right\rangle\right) / \sqrt{2}$. Mediante esta inversão é possível expressar o estado incial da eq. (1.10) em termos dos auto-estados do hamiltoniano de interação que formam uma base, obtendo

$$
\left|\psi_{I}(0)\right\rangle=c_{D}\left|\psi_{D}\right\rangle+c_{A}\left|\psi_{A}\right\rangle,
$$

onde $c_{D}=c_{1} \cos \theta e^{-i \phi_{p}}-c_{2} \sin \theta e^{-i \phi_{c}}$ e $c_{A}=c_{1} \sin \theta e^{i \phi_{p}}+c_{2} \cos \theta e^{\phi_{c}}$. Dado que os coeficientes $c_{1}$ e $c_{2}$ podem ser complexos, associamos uma fase $\psi_{1}$ e $\psi_{2}$, respectivamente, para cada um deles. Esses coeficientes estão fortemente ligados à probabilidade de popular esse estado mediante bombeamento ou emissão espontânea. Vamos ver o que acontece como o coeficiente $c_{A}$ em relação às diferenças de fase $\Delta \psi$ e $\Delta \phi$ a partir da definição

$$
c_{A}=c_{1} \sin \theta\left(1+\frac{\left|c_{2}\right|}{\left|c_{1}\right|} \frac{\Omega_{p}}{\Omega_{c}} e^{i(\Delta \phi-\Delta \psi)}\right) ; \quad \Delta \phi=\phi_{p}-\phi_{c} . \Delta \psi=\psi_{2}-\psi_{1} .
$$

Se considerarmos o acoplamento entre os dois campos e os átomos de forma balanceada (usualmente isso acontece no experimento, para obter um espectro resolvido), e assumirmos que a população $c_{1}$ e $c_{2}$ são muito próximas, então é razoável impor a condição

$$
\frac{\Omega_{c}}{\Omega_{p}}=\frac{\left|c_{2}\right|}{\left|c_{1}\right|}
$$


portanto, o coeficiente de acoplamento $c_{A}$ é expresso como

$$
c_{A}=c_{1} \sin \theta\left(1+e^{i(\Delta \phi-\Delta \psi)}\right),
$$

possui assim uma forma típica de interferência. Dependendo da fase em que bombeamos a população dos níveis, temos um estado puramente escuro ou uma superposição deste com o estado de acoplamento. Quando o bombeamento de população possui uma diferença de fase $\Delta \phi-\Delta \psi=k \pi$, sendo $k \in \mathbb{Z}$, obtemos uma interferência destrutiva, sendo $c_{A}=0$ e $c_{D}=e^{i\left(k \pi+\phi_{2}\right)}$ (levando em conta a normalização na eq. (1.10)), mostrando assim que o estado do sistema é o estado escuro. Nesta condição de interferência destrutiva, observamos transparência induzida eletromagnéticamente. Se a diferença de fase é $\Delta \phi-\Delta \psi=2 k \pi$, temos uma interferência construtiva, que leva o sistema ao estado de acoplamento $\left|\psi_{A}\right\rangle$.

Se de alguma forma conseguimos preparar o sistema no estado escuro, então pela evolução temporal dada na eq. (1.8), sendo $\left|\psi_{D}\right\rangle$ um auto-estado do hamiltoniano de interação com auto valor $\lambda_{D}=0$, o estado do sistema para um tempo $t$ vai continuar sendo o estado escuro com exceção de uma fase

$$
\left|\psi_{I}(t)\right\rangle=e^{\frac{i H_{\text {int }}^{\prime} t}{\hbar}}\left|\psi_{D}\right\rangle=e^{i\left(k \pi+\phi_{2}\right)}\left|\psi_{D}\right\rangle
$$

Isto é conhecido como aprisionamento coerente de população (CPT- Coherent Population Trapping). Mas não é o único caso onde poderiamos ter CPT. Podemos analisar o caso quando o estado se encontra no estado de acoplamento, ou seja a difereça de fase é $\Delta \phi-\Delta \psi=2 k \pi$ e portanto $c_{D}=0$ e $c_{A}=1$. Apesar do estado $\left|\psi_{A}\right\rangle$ para tempo $t=0$ também é uma superposição dos estados fundamentais $|1\rangle$ e $|2\rangle$ em relação ao estado escuro, sua dinâmica não leva a um aprisionamento de população direta como na eq. (1.15), já que

$$
\left|\psi_{I}(t)\right\rangle=e^{\frac{i H_{\text {int }}^{\prime} t}{\hbar}}\left|\psi_{A}\right\rangle=\frac{1}{\sqrt{2}}\left(e^{i \Omega t}\left|\psi_{+}\right\rangle+e^{-i \Omega t}\left|\psi_{-}\right\rangle\right),
$$

na base do hamiltoniano $H_{o}$ obtemos

$$
\left|\psi_{I}(t)\right\rangle=e^{\frac{i H_{\text {int }}^{\prime} t}{\hbar}}\left|\psi_{A}\right\rangle=i \sin (\Omega t)|3\rangle+\cos (\Omega t)\left|\psi_{A}\right\rangle .
$$

sendo esta uma oscilação de Rabi com frequência $\Omega$. Com este resultado, mostramos que existe a possibilidade dos átomos serem excitados para o $|3\rangle$, com máxima população neste estado para tempos $\Omega t=n \pi / 2 \operatorname{com} n \in \mathbb{N}$, e portanto não teremos transparência.

Finalmente se considerarmos o estado inicial geral da eq. (1.10), mediante a eq. (1.8), achamos que a evolução temporal do sistema é

$$
\left|\psi_{I}(t)\right\rangle=c_{D}\left|\psi_{D}\right\rangle+c_{A}\left(i \sin (\Omega t)|3\rangle+\cos (\Omega t)\left|\psi_{A}\right\rangle\right) .
$$


Como poderia o sistema evoluir até o estado escuro? Poderíamos ver o aprisionamento coerente de população a partir da eq (1.18)? A resposta é não. Inicialmente estamos considerando o sistema como um sistema fechado, e se $c_{A} \neq 0$, então sempre teremos a condição de fase do bombeamento $\Delta \phi-\Delta \psi \neq k \pi$ e, portanto, o sistema não poderá estar aprisionado no estado escuro. No entanto, se introduzirmos, de forma fenomenológica, as taxas de decaimento $\Gamma_{3 A}$ e $\Gamma_{3 D}$, poderemos obervar que, embora exista uma dinâmica cíclica entre o estado acoplado $\left|\psi_{A}\right\rangle$ e o estado excitado $|3\rangle$, como é mostrado na figura (1.2), considerando a possibilidade do decaimento do estado exitado para o estado puramente escuro $\left|\psi_{D}\right\rangle$, de acordo com a eq.(1.15), o sistema não poderá sair do estado escuro e, portanto, teremos aprisionamento de população, embora o estado incial seja arbitrário.

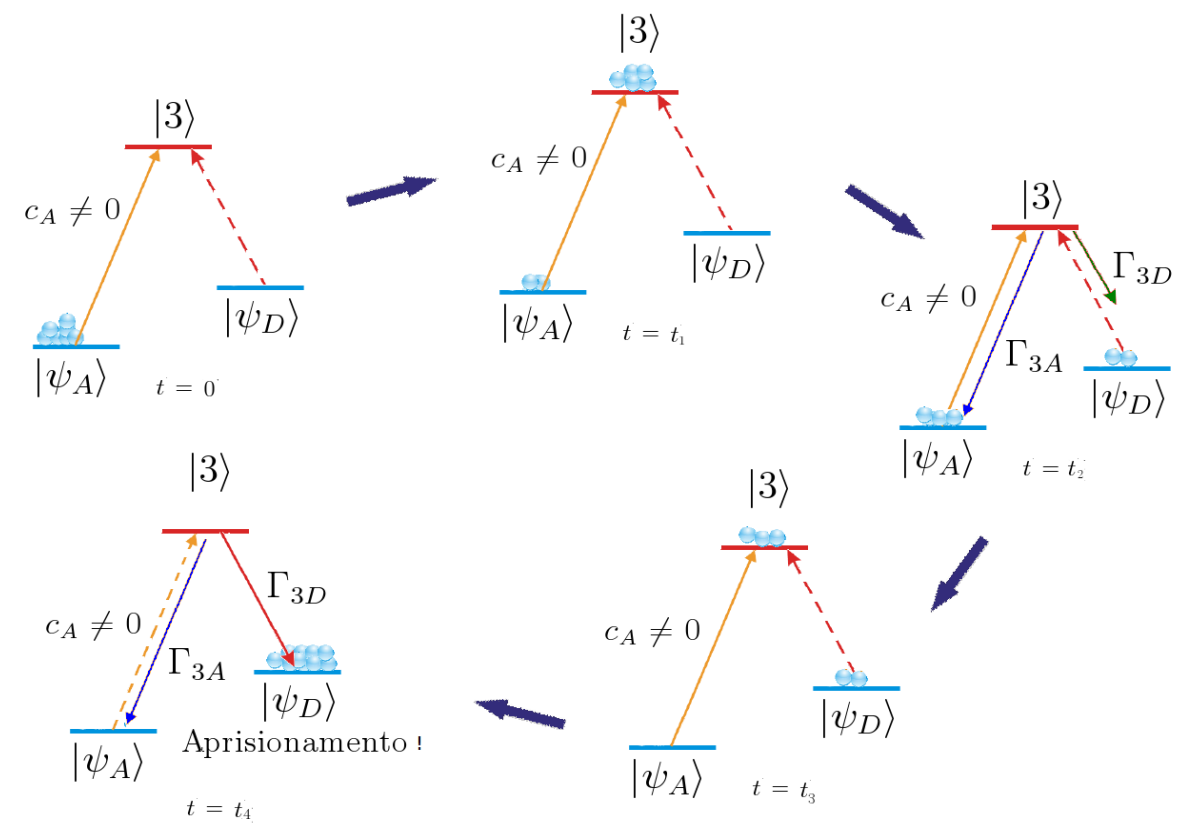

Figura 1.2: Ciclo dinâmico do sistema aberto. Consideramos o sistema inicialmente no estado $\left|\psi_{A}\right\rangle$ para tempo $t=0$. Ele evolui para o estado excitado $|3\rangle$ em $t=t_{1}$. Num tempo $t=t_{2}$ parte da população pode decair para o estado $\left|\psi_{A}\right\rangle$ numa taxa $\Gamma_{3 A}$, existindo a possibilidade de uma parte da população decair no estado escuro $\left|\psi_{D}\right\rangle$ numa taxa $\Gamma_{3 D}$, proporcional à taxa de bombeio $\Omega$. A população restante é excitada de novo para o estado $|3\rangle$ no tempo $t=t_{3}$. O processo se repete sucessivamente até que toda população se acumula no estado escuro $\left|\psi_{D}\right\rangle$ num tempo dado $t=t_{4}$, ficando aprisionado ali.

Como podemos observar, foi possível responder à pergunta inicial sobre como o sistema efetivamente poderia evoluir para o estado escuro mantendo-se aprisionado nele, introduzindo a noção de emissão espontânea dos átomos. No entanto, podemos usar o formalismo da Equação Mestra, para analisar o sistema como um sistema aberto, que interage com seu entorno, retomando os termos de emissão es- 
pontânea. Inclusive, nesse formalismo, podemos considerar a dessintonia do campo eletromagnético com respeito às transições atômicas, permitindo um tratamento mais completo da dinâmica do sistema.

\subsection{EIT em sistemas de 3 níveis}

O sistema é descrito no tempo pelo operador de densidade $\hat{\rho}(t)$, e a evolução temporal é determinada pela Equação Mestra com a qual obtemos as equações de Bloch. Considerando o hamiltoniano da eq. (1.1), a equação Mestra em [35] é definida como

$$
\begin{aligned}
\frac{d \hat{\rho}}{d t}= & -\frac{i}{\hbar}\left[H_{o}+H_{\mathrm{int}}, \hat{\rho}\right]+\frac{\Gamma_{31}}{2}\left[2 \hat{\sigma}_{13} \hat{\rho} \hat{\sigma}_{31}-\hat{\sigma}_{33} \hat{\rho}-\hat{\rho} \hat{\sigma}_{33}\right] \\
& +\frac{\Gamma_{32}}{2}\left[2 \hat{\sigma}_{23} \hat{\rho} \hat{\sigma}_{32}-\hat{\sigma}_{33} \hat{\rho}-\hat{\rho} \hat{\sigma}_{33}\right] \\
& +\gamma_{21}\left[2 \hat{\sigma}_{21} \hat{\rho} \hat{\sigma}_{12}-\hat{\sigma}_{22} \hat{\rho}-\hat{\rho} \hat{\sigma}_{22}\right] \\
& +\gamma_{3}\left[2 \hat{\sigma}_{33} \hat{\rho} \hat{\sigma}_{33}-\hat{\sigma}_{33} \hat{\rho}-\hat{\rho} \hat{\sigma}_{33}\right]
\end{aligned}
$$

onde o primero termo da direita determina a dinâmica do sistema como um sistema fechado, sendo esta a conhecida equação quântica de Liouville. Os três termos seguintes são os termos de Lindblad, que representam o efeito de relaxação das populações e coerências através das taxas de decaimento radiativo nas equações de movimento. O último termo representa o tempo de interação da luz com os átomos (no caso da célula de vapor, os átomos interagem com a luz enquanto atravessam a seção transversal do feixe), ou o controle de população (como acontece com átomos

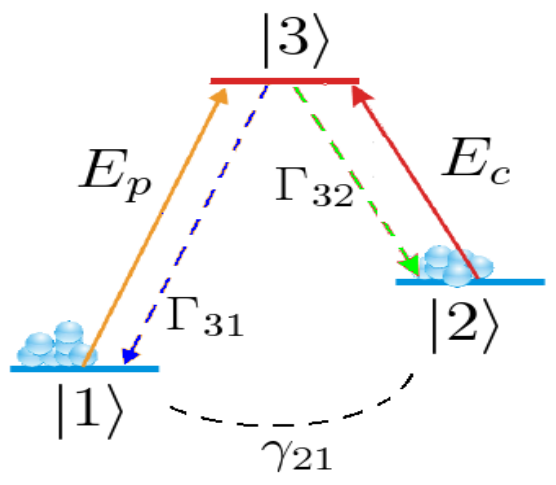

Figura 1.3: Esquema de níveis do sistema de três níveis. Cada transição é acoplada com os campos $\mathbf{E}_{p}$ e $\mathbf{E}_{c}$, para os quais temos as taxa de decaimento $\Gamma_{31}$ e $\Gamma_{32}$, respectivamente. No caso geral consideramos uma taxa de decoerencia $\gamma_{21}$ entre os níveis fundamentais. 
frios, em que é necessário evitar decaimentos para outros níveis de momento angular). As taxas $\Gamma_{31}$ e $\Gamma_{32}$ correspondem ao possível decaimento do nivel $|3\rangle$ para os dois estados fundamentais $|1\rangle$ e $|2\rangle$, respectivamente, como mostra a figura 1.3. Os últimos dois termos entrarão na teoria de forma fenomenológica, porque não correspondem a uma transição ótica, proibida por paridade. Porém a taxa $\gamma_{21}$ indica que existe a possibilidade da população do estado $|2\rangle$ ter perdas lentas no tempo, com respeito à taxa de emissão espontânea $\Gamma_{i j}$, gerando decoerência na superposição de estados fundamentais do estado escuro. Essa taxa de decorência pode ter origem na colisão entre átomos e também no tempo finito de interação entre os átomos e o laser.

Vamos considerar átomos frios no sistema que estudaremos. Portanto, é possível desprezar o termo de decoerência $\gamma_{3}$, porque o tempo de interação dos átomos com o feixe de luz, é muito maior que as frequências de Rabi $\Omega_{p}$ e $\Omega_{c}$, e a taxa de emissão espontânea $\Gamma$. Levando em conta isso, obtemos as equações de Bloch dos elementos $\rho_{i j}=\langle i|\hat{\rho}| j\rangle$ do operador densidade, a partir da equação mestre (1.19), de modo que

$$
\begin{aligned}
\dot{\rho}_{11}= & i \Omega_{p} \rho_{13} e^{-i\left(\omega_{p} t+\phi_{p}\right)}-i \Omega_{p} \rho_{31} e^{i\left(\omega_{p} t+\phi_{p}\right)}+\Gamma_{31} \rho_{33}, \\
\dot{\rho}_{22}= & i \Omega_{c} \rho_{23} e^{-i\left(\omega_{c} t+\phi_{c}\right)}-i \Omega_{c} \rho_{32} e^{i\left(\omega_{c} t+\phi_{c}\right)}+\Gamma_{32} \rho_{33}, \\
\dot{\rho}_{33}= & -i \Omega_{p} \rho_{13} e^{-i\left(\omega_{p} t+\phi_{p}\right)}+i \Omega_{p} \rho_{31} e^{i\left(\omega_{p} t+\phi_{p}\right)}-i \Omega_{c} \rho_{23} e^{-i\left(\omega_{c} t+\phi_{c}\right)} \\
& +i \Omega_{c} \rho_{32} e^{i\left(\omega_{c} t+\phi_{c}\right)}-\left(\Gamma_{31}+\Gamma_{32}\right) \rho_{33}, \\
\dot{\rho}_{13}= & -i \omega_{31} \rho_{13}-i \Omega_{p}\left(\rho_{33}-\rho_{11}\right) e^{i\left(\omega_{p} t+\phi_{p}\right)}+i \Omega_{c} \rho_{12} e^{i\left(\omega_{c} t+\phi_{c}\right)} \\
& -\frac{\left(\Gamma_{31}+\Gamma_{32}+2 \gamma_{3}\right)}{2} \rho_{13}, \\
\dot{\rho}_{23}= & -i \omega_{32} \rho_{23}-i \Omega_{c}\left(\rho_{33}-\rho_{22}\right) e^{i\left(\omega_{c} t+\phi_{c}\right)}+i \Omega_{p} \rho_{21} e^{i\left(\omega_{p} t+\phi_{p}\right)} \\
& -\frac{\left(\Gamma_{31}+\Gamma_{32}+2 \gamma_{3}\right)}{2} \rho_{23}, \\
\dot{\rho}_{12}= & -i\left(\omega_{31}-\omega_{32}\right) \rho_{12}-i \Omega_{p} \rho_{32} e^{i\left(\omega_{p} t+\phi_{p}\right)}+i \Omega_{c} \rho_{13} e^{-i\left(\omega_{c} t+\phi_{c}\right)}-\gamma_{21} \rho_{12},
\end{aligned}
$$

onde $\omega_{i j}=\omega_{i}-\omega_{j}$ sendo $\omega_{i}$ a frequência definida no hamiltoniano livre. Na prática (e para simplificar) podemos considerar as taxas de decaimento $\Gamma_{31}=\Gamma_{32}=\Gamma$. Levando em conta as equações temporais do conjugado das coerências $\rho_{j i}=\rho_{i j}^{*} \mathrm{e}$ que $\operatorname{Tr}[\hat{\rho}]=1$, o conjunto de equações é completo.

Vamos introduzir agora as variáveis do sistema referencial girante, o que permite 
descrever as equações de Bloch independentes do tempo:

$$
\begin{aligned}
\rho_{i i} & \rightarrow \rho_{i i}, \\
\rho_{13} & \rightarrow e^{-i\left(\omega_{p} t+\phi_{p}\right)} \tilde{\rho}_{13}, \\
\rho_{23} & \rightarrow e^{-i\left(\omega_{c} t+\phi_{c}\right)} \tilde{\rho}_{23}, \\
\rho_{12} & \rightarrow e^{i\left[\left(\omega_{c}-\omega_{p}\right) t+\left(\phi_{p}-\phi_{c}\right)\right]} \tilde{\rho}_{12},
\end{aligned}
$$

A partir desta mudança de variáveis, podemos chegar ao conjunto de equações de Bloch independentes do tempo ${ }^{2}$,

$$
\begin{aligned}
& \dot{\tilde{\rho}}_{11}=i \Omega_{p}\left(\tilde{\rho}_{13}-\tilde{\rho}_{31}\right)+\Gamma \tilde{\rho}_{33} \\
& \dot{\tilde{\rho}}_{22}=i \Omega_{c}\left(\tilde{\rho}_{23}-\tilde{\rho}_{32}\right)+\Gamma \tilde{\rho}_{33} \\
& \dot{\tilde{\rho}}_{33}=-i \Omega_{p}\left(\tilde{\rho}_{13}-\tilde{\rho}_{31}\right)-i \Omega_{c}\left(\tilde{\rho}_{23}-\tilde{\rho}_{32}\right)-2 \Gamma \tilde{\rho}_{33} \\
& \dot{\tilde{\rho}}_{13}=-\left(i \delta_{1}+\Gamma\right) \tilde{\rho}_{13}-i \Omega_{p}\left(\tilde{\rho}_{33}-\tilde{\rho}_{11}\right)+i \Omega_{c} \tilde{\rho}_{12} \\
& \dot{\tilde{\rho}}_{32}=-\left(i \delta_{2}+\Gamma\right) \tilde{\rho}_{32}+i \Omega_{c}\left(\tilde{\rho}_{33}-\tilde{\rho}_{22}\right)-i \Omega_{p} \tilde{\rho}_{12} \\
& \dot{\tilde{\rho}}_{12}=-\left(i \delta_{R}+\gamma_{21}\right) \tilde{\rho}_{12}-i \Omega_{p} \tilde{\rho}_{32}+i \Omega_{c} \tilde{\rho}_{13}
\end{aligned}
$$

onde $\delta_{1}=\omega_{31}-\omega_{p}, \delta_{2}=\omega_{32}-\omega_{c}$, e a dessintonia Raman definida como $\delta_{R}=\delta_{1}-\delta_{2}$ da qual vai depender fortemente a absorção dos dois campos. A absorção do campo de prova, devida ao meio atômico (considerando o modelo de mostra fina, ou seja, a polarização de um átomo vezes o número de átomos que temos no meio), pode ser calculada a partir da relação entre o valor esperado da polarização do meio atômico (assumindo-a no eixo z para simplificar), dada por

$$
\vec{P}_{E_{p}}=\frac{\sum_{i}\left\langle\mathbf{d}_{\mathbf{1 3} i}\right\rangle}{V}=\frac{N_{a t o m}}{V} d_{13} \tilde{\rho}_{13} \hat{\mathbf{z}}
$$

tal que, em termos do campo de prova $\vec{E}_{p}$ (que está incluso na frequência de Rabi $\left.\Omega_{p}\right)$ a polarização da primeira ordem é $P_{E_{p}}=\epsilon_{o} \chi^{(1)} E_{p}$, onde a constante de proporcionalidade é a susceptibilidade elétrica. A dispersão e absorção da luz são associadas a $\Re e\left\{\chi^{(1)}\right\}$ e $\Im m\left\{\chi^{(1)}\right\}$, respectivamente.

Para solucionar o sistema de equações (1.30)-(1.35) de forma completa, ou seja, em qualquer condição das intensidades dos campos, é necessário resolver numericamente de forma geral. No entanto, para dar uma expressão analítica da susceptiblidade e desenvolver a intuição sobre o sistema, podemos considerar um acoplamento muito forte do campo de controle em relação ao campo de prova, ou seja, $\left|\Omega_{c}\right| \gg\left|\Omega_{p}\right|$,

\footnotetext{
${ }^{2}$ Neste caso não estamos considerando uma fase estocástica $\phi(t)$ nos campos e por isso podemos construir estas equações indepedentes do tempo de forma direta. Mas na descrição do modelo de difusão de fase para o ruido dos campos electromagnéticos, devemos ter um tratamento diferente.
} 
daí podemos assumir que toda a população fica no estado $|1\rangle$, de modo que $\rho_{11} \approx 1$ e $\rho_{22} \approx \rho_{33} \approx 0$. Usando as eqs. (1.33) - (1.35) obtemos uma solução para as coerências no regime estacionário:

$$
\begin{aligned}
& \tilde{\rho}_{32}=-\frac{i \Omega_{p}}{i \delta_{2}+\Gamma} \tilde{\rho}_{12} \\
& \tilde{\rho}_{12}=\frac{i \Omega_{c}\left(i \delta_{2}+\Gamma\right)}{\left(i \delta_{R}+\gamma\right)\left(i \delta_{2}+\Gamma\right)+\Omega_{p}^{2}} \tilde{\rho}_{13}
\end{aligned}
$$

tal que a coerência acoplada ao campo de prova tem como solução

$$
\tilde{\rho}_{13}=i\left[\frac{\left(i \delta_{R}+\gamma\right)\left(i \delta_{2}+\Gamma\right)+\Omega_{p}^{2}}{\left(i \delta_{1}+\Gamma\right)\left(i \delta_{R}+\gamma\right)\left(i \delta_{2}+\Gamma\right)+\Omega_{c}^{2}\left(i \delta_{2}+\Gamma\right)}\right] \Omega_{p} .
$$

Usando a expressão da polarização (1.36) e lembrando que $\Omega_{p}=\vec{d}_{13} \cdot \vec{E}_{p} / \hbar$, obtemos a susceptibilidade $\chi$

$$
\begin{aligned}
\vec{P}_{p}= & i \frac{\left|d_{13}\right|^{2} \varrho}{\epsilon_{o} \hbar}\left[\frac{\left(i \delta_{R}+\gamma\right)}{\left(i \delta_{1}+\Gamma\right)\left(i \delta_{R}+\gamma\right)+\Omega_{c}^{2}}\right] E_{p} \\
& +i \frac{\left|d_{13}\right|^{2} \varrho}{\epsilon_{o} \hbar}\left[\frac{\Omega_{p}^{2}}{\left(i \delta_{1}+\Gamma\right)\left(i \delta_{R}+\gamma\right)\left(i \delta_{2}+\Gamma\right)+\Omega_{c}^{2}\left(i \delta_{2}+\Gamma\right)},\right] E_{p}
\end{aligned}
$$

onde $\varrho=N_{\text {atom }} / V$. O primeiro termo mostra a resposta linear da polarização com o campo, e o segundo termo corresponde à resposta não linear do meio atômico. A partir do primeiro termo podemos mostrar a susceptibilidade em primeira ordem como $\chi^{(1)}=\Re e\left\{\chi^{(1)}\right\}+i \Im m\left\{\chi^{(1)}\right\}$, de modo que a dispersão é dada por

$$
\Re e\left\{\chi^{(1)}\right\}=\frac{\left|d_{13}\right|^{2} \varrho}{\epsilon_{o} \hbar}\left\{\frac{\delta_{1}\left(\gamma^{2}+\delta_{R}^{2}\right)-\Omega_{c}^{2} \delta_{R}}{\left|\left(i \delta_{1}+\Gamma\right)\left(i \delta_{R}+\gamma\right)+\Omega_{c}^{2}\right|^{2}}\right\},
$$

e a absorção por

$$
\Im m\left\{\chi^{(1)}\right\}=\frac{\left|d_{13}\right|^{2} \varrho}{\epsilon_{o} \hbar}\left\{\frac{\gamma \Omega_{c}^{2}+\Gamma\left(\gamma^{2}+\delta_{R}^{2}\right)}{\left|\left(i \delta_{1}+\Gamma\right)\left(i \delta_{R}+\gamma\right)+\Omega_{c}^{2}\right|^{2}}\right\} .
$$

Considerando o caso no qual não temos decorência nos níveis fundamentais, $\gamma=0$, temos a expressão mais simples da absorção

$$
\Im m\left\{\chi^{(1)}\right\}=\frac{\left|d_{13}\right|^{2} \varrho}{\epsilon_{o} \hbar}\left\{\frac{\Gamma \delta_{R}^{2}}{\left|\delta_{R}\left(i \Gamma-\delta_{1}\right)+\Omega_{c}^{2}\right|^{2}}\right\},
$$

Na figura 1.4.a mostramos a absorção para várias frequências de Rabi do campo de controle, considerando as decoerências na superposição dos estados fundamentais, como foi determinado na eq. (1.44). A figura mostra que conforme aumentamos a 

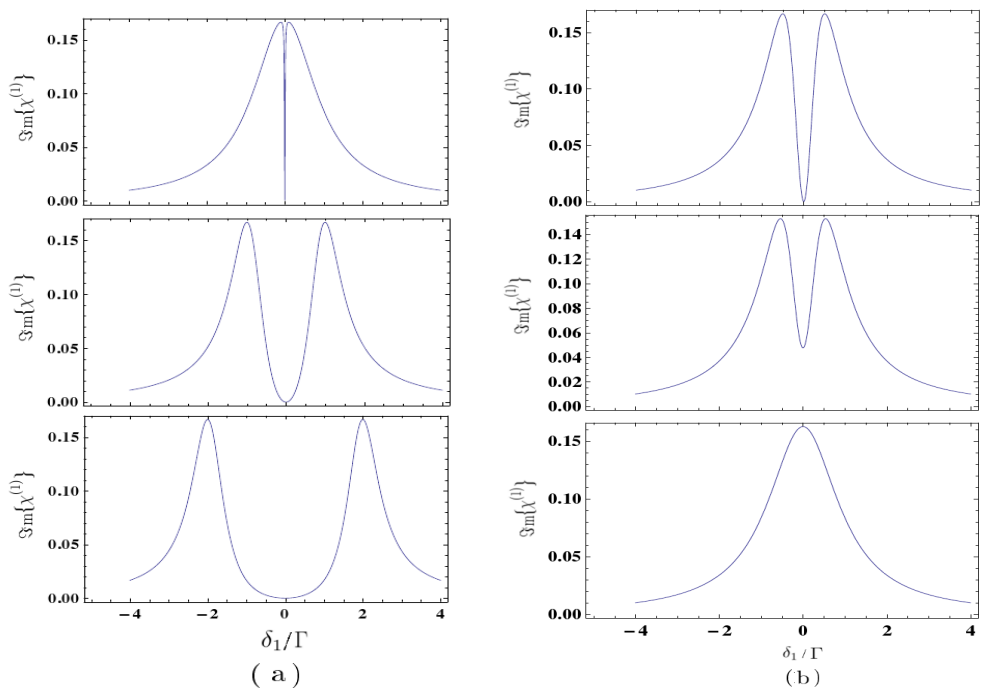

Figura 1.4: Absorção do campo de prova em função da dessintonia $\delta_{1}$, em condição de EIT. (a) Sem decorência $\gamma=0$ e para as frequências de Rabi $\Omega_{c}=0.1 \Gamma, \Omega_{c}=\Gamma$ e $\Omega_{c}=2 \Gamma$. (b) Com decoerência para valores de $\gamma=0, \gamma=0.1 \Gamma$ e $\gamma=10 \Gamma$, com $\Gamma=6$ e frequência de Rabi $\Omega_{p}=0.5 \Gamma$

frequência de Rabi $\Omega_{c}$, a janela de transparência é mais larga, ou seja, temos um alargamento por potência. Para uma potência $\Omega_{c}=2 \Gamma$, temos um perfil que poderia ser reproduzido por duas transições próximas com perfil Lorentziano. No entanto para uma frequência de Rabi menor $\Omega_{c}=0.1 \Gamma$ temos uma janela de transparência muito fina, o que mostra que não pode ser vista como duas transições proximas, senão como o fenômeno de interferência perto da ressonância, cuja largura é menor que a largura natural.

Por outro lado, na figura 1.4.b, mostramos a absorção de acordo com a eq.(1.43), onde é levada em conta a taxa de decoerência ${ }^{3} \gamma$. Nesta figura observamos a transição de transparência total a uma perda de transparência, voltando ao caso de absorção da luz no sistema de dois níveis. No primeiro caso, a taxa de emissão espontânea é maior que a taxa de coerência (quando $\Gamma=6$ e $\gamma=0$ ), ou seja, o sistema pode fazer um ciclo que oscila entre o estado $\left|\psi_{A}\right\rangle$ e o estado $|3\rangle$ (como foi visto na figura 1.2) e pela rápida taxa de emissão espontânea $\Gamma$ pode decair no estado escuro, permanecendo nele durante tempo sufuciente antes de que no tempo de decoerência $1 / \gamma$ quebre-se a superposição do estado escuro, induzindo assim, transparência. No entanto, no caso em que a taxa de decoerência é maior que o a taxa de emissão espontânea (quando $\Gamma=6$ e $\gamma=10 \Gamma$ ), acontece da população do estado $|2\rangle$ decair mais rápido para o estado $|1\rangle$ em relação ao tempo de emissão espontânea. Portanto, o sistema não possui um estado escuro no qual chegaria mediante emissão

\footnotetext{
${ }^{3}$ Por simplicidade, denominamos a taxa $\gamma_{21}$ como a taxa $\gamma$
} 
espontanea, anulando qualquer possibilidade de interferência e retomando assim o perfil de absorção de dois níveis.

Contudo, o campo de prova em geral pode não ter uma intensidade muito menor que o campo de controle, definindo condições sobre as populações como foi feito no começo do análise anterior. Em geral, nas experiências feitas no laboratório, a intensidade do campo de prova pode ser muito próxima da intensidade do campo de controle. Portanto, para determinar a solução geral das populações e as coerências atômicas das eqs. (1.30)-(1.32), precisamos definir a nova matriz $w=\tilde{\rho}-\tilde{\rho_{33}} \mathbb{I}$ na qual a dinâmica das componentes $w_{11}$ e $w_{22}$ são dadas por

$$
\begin{aligned}
& \dot{w}_{11}=2 i \Omega_{p}\left(\tilde{\rho}_{13}-\tilde{\rho}_{31}\right)+i \Omega_{c}\left(\tilde{\rho}_{23}-\tilde{\rho}_{32}\right)+\Gamma\left(w_{11}+w_{22}\right)-\Gamma, \\
& \dot{w}_{22}=2 i \Omega_{c}\left(\tilde{\rho}_{23}-\tilde{\rho}_{32}\right)+i \Omega_{p}\left(\tilde{\rho}_{13}-\tilde{\rho}_{31}\right)+\Gamma\left(w_{11}+w_{22}\right)-\Gamma .
\end{aligned}
$$

Estas equações, juntamente com o sistema de eqs. (1.30)-(1.35), podem ser representadas de forma matricial, tal que

$$
\frac{d w}{d t}=\mathbf{A} \cdot w+w_{0}
$$

onde $w_{0}$ é definido como a matriz de elementos $w_{0_{11}}=-\Gamma, w_{0_{22}}=-\Gamma$ e $w_{0_{i j}}=0$ para $i, j \neq 1,2$. A matriz $\mathbf{A}$ é definida como

$$
\mathbf{A}=\left(\begin{array}{cccccccc}
\Gamma & \Gamma & 2 i \Omega_{p} & -2 i \Omega_{p} & i \Omega_{c} & -i \Omega_{c} & 0 & 0 \\
\Gamma & \Gamma & i \Omega_{p} & -i \Omega_{p} & 2 i \Omega_{p} & -2 i \Omega_{p} & 0 & 0 \\
-i \Omega_{p} & 0 & -\left(i \delta_{1}+\Gamma\right) & 0 & 0 & 0 & i \Omega_{c} & 0 \\
i \Omega_{p} & 0 & 0 & \left(i \delta_{1}-\Gamma\right) & 0 & 0 & 0 & -i \Omega_{c} \\
0 & -i \Omega_{c} & 0 & 0 & -\left(i \delta_{2}+\Gamma\right) & 0 & 0 & i \Omega_{p} \\
0 & i \Omega_{c} & 0 & 0 & 0 & \left(i \delta_{2}-\Gamma\right) & -i \Omega_{p} & 0 \\
0 & 0 & i \Omega_{c} & 0 & 0 & -i \Omega_{p} & -\left(i \delta_{R}+\gamma_{21}\right) & 0 \\
0 & 0 & 0 & -i \Omega_{c} & i \Omega_{p} & 0 & 0 & \left(i \delta_{R}-\gamma_{21}\right)
\end{array}\right) .
$$

Se consideramos o regime estacionário do sistema, teremos que $\frac{d w}{d t}=0$, portanto, mediante a inversa da matriz A podemos determinar a solução para as populações e coerências do sistema a partir de

$$
w_{\mathrm{est}}=-\left(\mathbf{A}^{-1}\right) w_{0} .
$$

Usando o elemento $\rho_{13}^{\text {est }}$ da matriz $w_{\text {est }}$ e substituindo na polarização da eq.(1.36), podemos obter a absorção em função das dessintonias dos campos. No entanto, é possível resolver o sistema de equações e achar uma solução para a absorção, mapeando a matriz de densidade num vetor do espaço de Liouville, formalismo que será explicado na próxima seção.

Na figura 1.5, mostramos a absorção de cada um dos feixes a partir da eq.(1.49) assumindo que não temos decoerência nos estados fundamentais $\gamma=0$. Para o caso em que consideramos $\Omega_{c} \gg \Omega_{p}$, a linha violeta da figura 1.5.a e 1.5.b reproduz os 
resultados obtidos anteriormente pela expressão analítica (1.44). No segundo caso em que consideramos $\Omega_{c} \approx \Omega_{p}$ (linha azul), que mostra a situação mais próxima ao caso experimental, os dois feixes são igualmente absorvidos. Na figura 1.5.c mostramos a dispersão dos campos mediante a parte real da susceptibilidade nas duas situações da frequência de Rabi $\Omega_{c}$. Finalmente na figura 1.5.d mostramos as populações no caso próximo ao caso experimental $\Omega_{c} \approx \Omega_{p}$, em que podemos observar que o estado excitado é minimamente populado em condição de EIT.
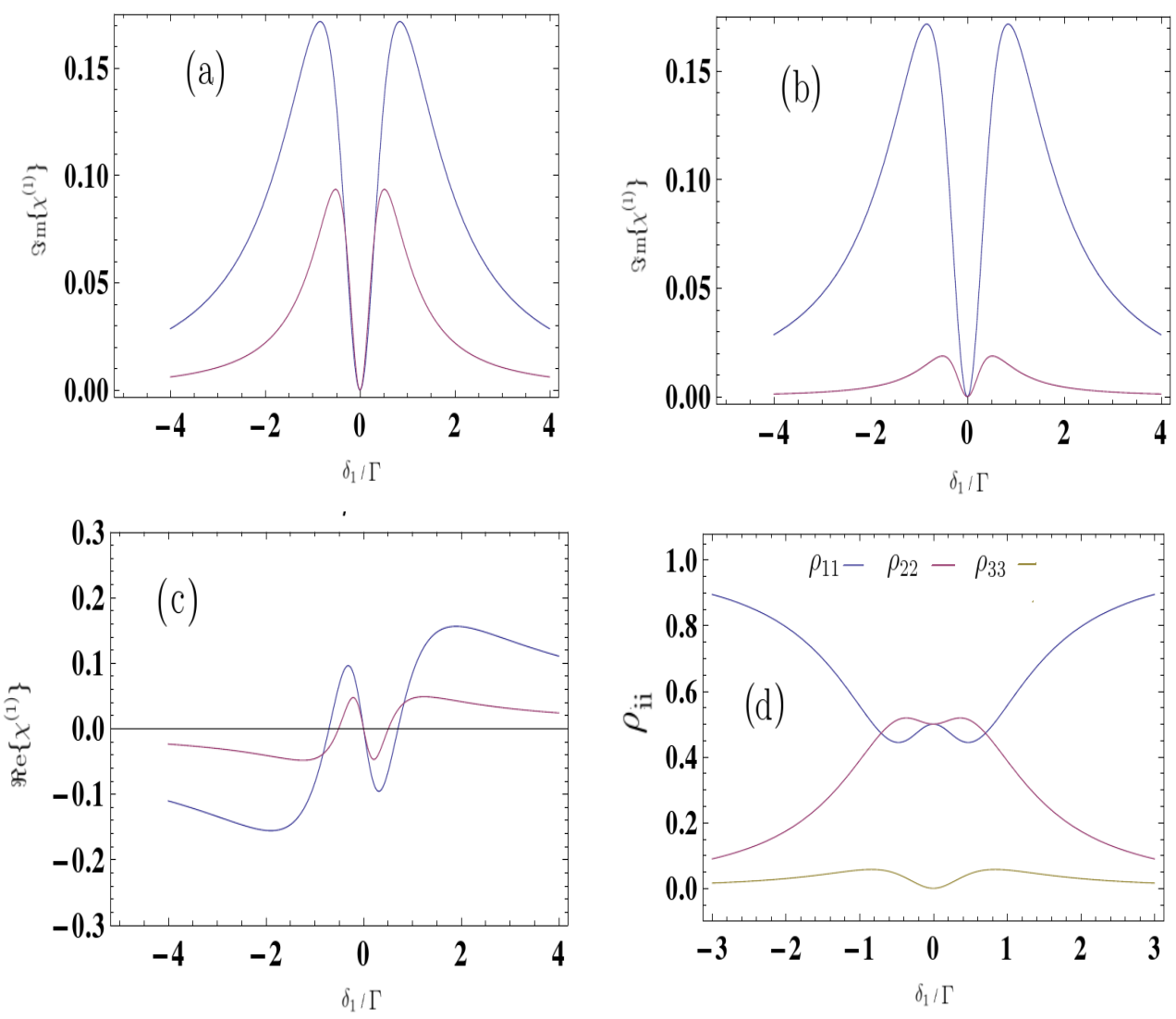

Figura 1.5: Comparação entre resultados das coerencias e populações, numa situação de acoplamento forte $\Omega_{c} \gg \Omega_{p}$ (linha violeta) e uma situação de acoplamento balanceado $\Omega_{c} \approx \Omega_{p}$ (linha azul). (a) Absorção do campo de prova. (b) Absorção do campo de controle. (c) Dispersão do campo de prova. (d) Populações atômicas no caso de acoplamento forte.

Se consideramos decoerência nos estados fundamentais, perdemos transparência no ponto de ressonância conforme a taxa $\gamma$ aumenta. Na figura 1.6.a comparamos o perfil de absorção no caso em que $\gamma=0$ (linha violeta) e $\gamma=0.2$ (linha azul). Da mesma forma, mostramos a dispersão na figura 1.6.b, onde vemos que a inclinação da refração diminui perto da ressonância, retomando o perfil de dispersão do sistema 
de dois níveis.
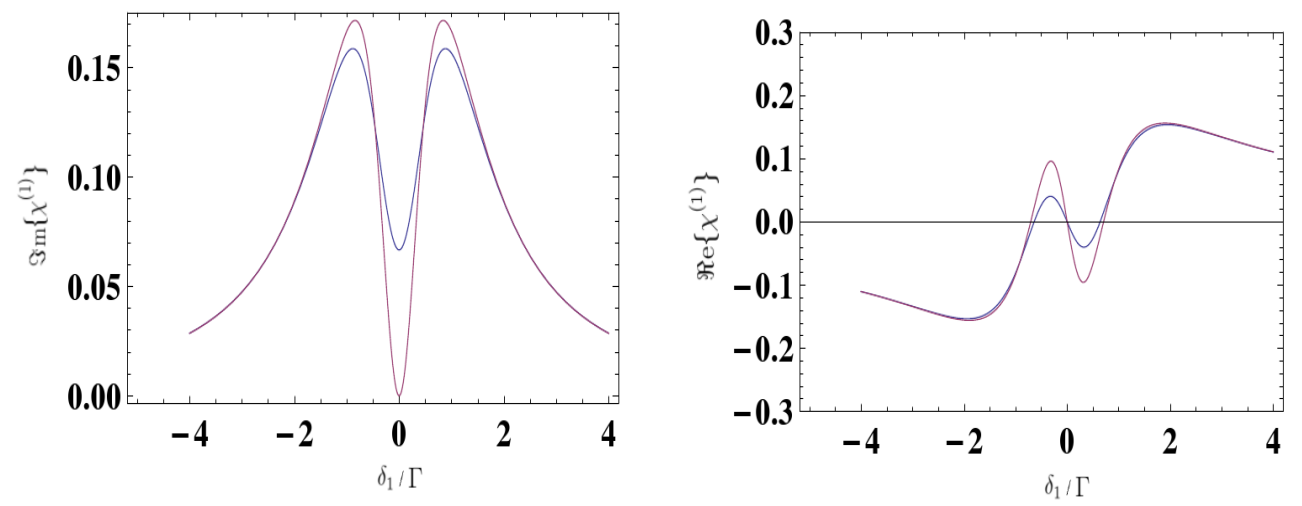

Figura 1.6: (a) Absorção do campo de prova, sem decoerência $\gamma=0$ (linha violeta) e com decoerência $\gamma=0.2 \Gamma$ (linha azul), em condição de $\Omega_{c} \gg \Omega_{p}$. (b) Disperção do campo de prova, sem decoerência $\gamma=0$ (linha violeta) e com decoerência $\gamma=0.2 \Gamma$ (linha azul), em condição de $\Omega_{c} \approx \Omega_{p}$

Até agora, estudamos o fenômeno da transparência induzida em um sistema atômico de três níveis (não degenerado), onde trabalhamos com campos linearmente polarizados de frequências distintas. No entanto, este não é o único sistema no qual podemos obervar EIT. Na próxima seção veremos como é possível ter EIT em sistemas de dois níveis degenerados. Será importante levar em conta a polarização dos campos para observar a interferência dos canais de bombeamento.

\subsection{EIT em sistemas de 2 níveis com degenerescências}

O fenômeno de EIT também pode ser observado quando os estados fundamentais do átomo são degenerados em energia, mantendo a configuração $\Lambda$, porém com momento angular diferente. Para acoplar oticamente essa classe de estados, é necessário usar polarizações circulares opostas, que eventualemente, pelos canais de excitação podem entrar em interferência destrutiva, induzindo assim EIT. Para observar EIT neste tipo de sistemas, temos duas situações: a primeira em que temos dois feixes de luz com polarizações circulares opostas e o controle das frequências para cada um deles é independente, como mostra a figura 1.7.a; a segunda situação é, mediante campos magnéticos, quebrarmos a degenerescência dos níveis, criando assim dessintonia dos feixes mediante o efeito de Hanle [38, 39], o meio atômico absorve preferencialmente as componentes circulares, como mostrado a figura 1.7.b.

As experiências feitas no laboratório implementam os dois esquemas, mostrando que a primeira situação reduz-se ao caso de sistema de três níveis, mostrando concordância com os resultados experimentais. No entanto, para o caso de Hanle-EIT, 
devemos usar o formalismo de interação de luz com átomo de dois níveis, porém levando em conta a interação com campos magnéticos, que simularão o efeito de dessintonia que tínhamos no caso anterior. Adicionalmente, os canais de relaxação do estado excitado para o estado fundamental são um pouco mais complexos que no caso dos três níveis. Estes serão descritos no final da seção.

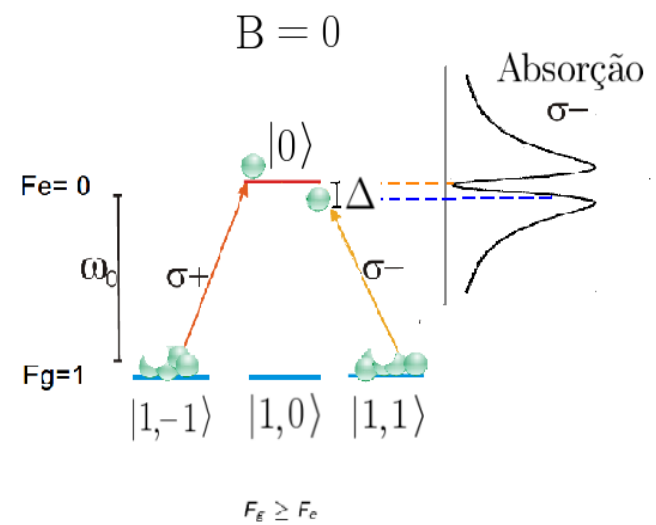

(a)

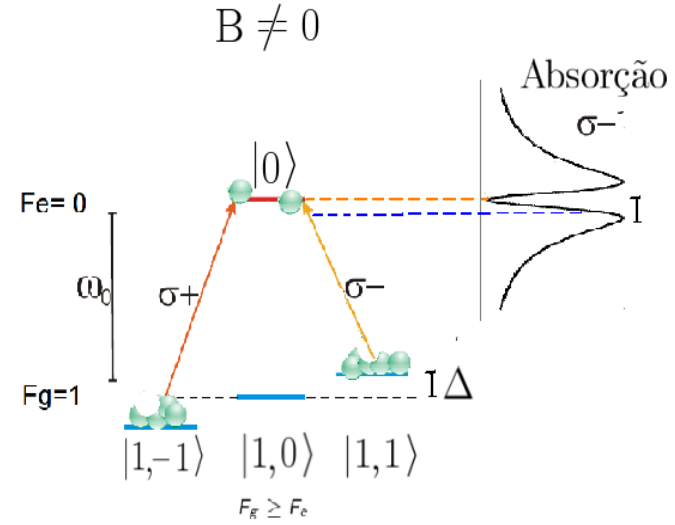

(b)

Figura 1.7: (a) EIT num sistema de dois níves degenerados com controle da frequência dos campos, independente. (b) Hanle-EIT em sistema de dois níveis degenerados, aplicando um campo magnético para criar dessintonia sintonizada nos dois níveis.

A degenerescência dos estados hiperfinos do Rubídio (que pode ter no mínimo 0 e máximo 9 estados), em geral dá lugar ao fenômeno de EIT e EIA, sendo este último absorção electromagnéticamente induzida que se dá quando o momento angular dos níveis satisfazem $F_{\text {excitado }}>F_{\text {fund }}$, tendo aumento de absorção na ressonância Raman [36, 37]. Mas este fenômeno está fora dos objetivos deste trabalho. No caso

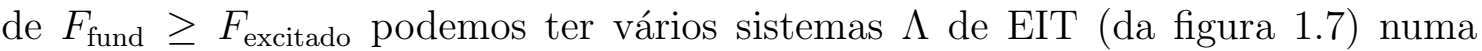
mesma transição de dois níveis.

A vantagem deste sistema de dois níveis degenerados em relação ao sistema de três níveis distintos é o fato de podermos usar um único laser, que deve manter sintonizada a frequência. Neste caso, qualquer tipo de dessintonia, pode ser feita facilmente mediante dispositivos de modulação acusto-ótica. Do ponto de vista prático, o caso de três níveis é mais dificil, porque precisamos de dois laseres com frequências relativamente distintas e sintonisadas nas duas transições. Além disso, é necessário manter os laser em fase, caso contrário o perfil de EIT é contaminado pela disfusão de fase entre os lasers.

O enfoque das experiências no laboratório envolveram a situação na qual, mesmo 
lidando com estados degenerados, o controle de frequências de cada campo com polarização circular oposta era independente. Manter uma compensação de campo magnético foi importante ${ }^{4}$ para evitar a quebra de degenerescência. Dadas estas condições, o sistema reduz-se ao sistema de três níveis, fazendo uma média sobre o número de sistemas $\Lambda$, em que usamos o formalismo que foi descrito na seção anterior. No entanto o formalismo das experiências com Hanle-EIT já foi estudado nas referências [30, 36, 29], de forma que nessa seção apresentaremos o formalismo de maneira mais esquemática.

Um sistema atômico de dois níveis degenerados consiste em um nível fundamental com momento angular $F_{f}$, cuja degenerescência de níveis Zeeman é $2 F_{f}+1$, e um estado excitado, com momento angular $F_{e}$, cuja degenerescência é $2 F_{e}+1$, como é mostrado na figura 1.8. Acoplando as transições com luz de polarização $\sigma_{+}$e $\sigma_{-}$, podemos observar múltiplas configurações tipo $\Lambda$ que podem dar lugar a EIT, únicamente no caso quando $F_{f} \geq F_{e}$.

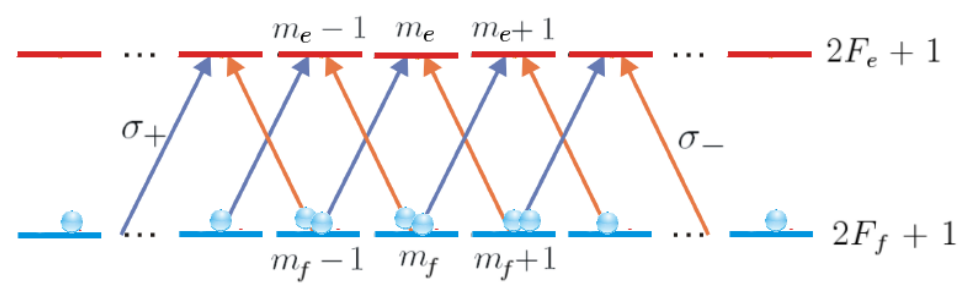

Figura 1.8: Esquema de níveis degenerados, dando lugar para vários sistemas de EIT em configuração $\Lambda$

A dinâmica do sistema é definida por, uma dinâmica livre dos átomos determinada pelo hamiltoniano $H_{o}$, uma interação com a radiação $H_{\text {Rad }}$ e com campos magnéticos definida por $H_{B}$. A evolução temporal do sistema é determinada pela equação de Bloch dada por

$$
\frac{d \hat{\rho}}{d t}=-\frac{i}{\hbar}\left[H_{o}+H_{\mathrm{Rad}}+H_{B}, \hat{\rho}\right]+\left.\frac{\partial \hat{\rho}}{\partial t}\right|_{\text {dis }},
$$

onde o último termo, corresponde à dissipação do sistema, devido principalmente ao efeito da degenerescência dos níveis. Esta dissipação é dada por

$$
\left.\frac{\partial \hat{\rho}}{\partial t}\right|_{\text {dis }}=-\frac{\Gamma}{2}\left\{P_{e}, \hat{\rho}\right\}+\Gamma\left(2 F_{e}+1\right) \sum_{q=-1,0,1}\left(P_{f} \hat{Q}^{\dagger q} P_{e}\right) \hat{\rho}\left(P_{e} \hat{Q}^{\dagger q} P_{f}\right)-\gamma\left(\rho-\rho_{0}\right) .
$$

onde $P_{e}$ e $P_{f}$ são o projetores dos estados excitados e fundamentais degenerados, respectivamente. Estes serão definidos em breve junto com o dipolo reduzido $\hat{Q}^{q}$.

\footnotetext{
${ }^{4}$ Estes detalhes são descritos no capitulo 3
} 
Se comparamos a eq.(1.19) que descreve a dinâmica do sistema de três níveis, com a eq.(1.50) que descreve a dinâmica para os sistemas de dois níveis, vemos que são muito parecidas. Os primeiros termos das duas equações são quase iguais, com exceção da presença de campo magnético descrito por $H_{B}$, para o sistema de dois níveis. Agora, se analisamos os dois primeiros termos dissipativos da eq.(1.19), caracterizados por $\Gamma_{31}$ e $\Gamma_{32}$, estes fazem o mesmo papel que o primeiro termo dissipativo da eq.(1.51) proporcional a $\Gamma / 2$, que descreve a dinâmca da população do estado excitado e das coerências. O termo que contribui para decoerência dos estados fundamentais, que depende de $\gamma_{21}$ para o sistema de três níveis, é equivalente ao segundo termo dissipativo $\Gamma\left(2 F_{e}+1\right)$ do sistema de dois níveis. O último termo de cada uma das equações representa a dissipação devida ao controle de população no caso de átomos frios, onde é feita uma transferência não perfeita da população dos estados usados para o resfriamento e os estados acoplados para observar EIT.

Mostraremos cada um dos termos que compõem o hamiltoniano total do sistema da eq. (1.50), descrevendo rapidamente o termo de átomo e sua interação com a radiação. Posteriormente, dedicaremos duas seções para descrever o termo de interação com campo magnético e de dissipação.

A primeira contribuição no hamiltoniano total é o termo que contribui para a evolução livre do átomo, dado por

$$
H_{o}=\hbar \omega_{f} \hat{P}_{f}+\hbar \omega_{e} \hat{P}_{e}
$$

onde os operadores de projeção $\hat{P}_{f}$ e $\hat{P}_{e}$ levam em conta as degenerescências de cada um dos níveis, ou seja

$$
\hat{P}_{f}=\sum_{m_{f}}\left|F_{f}, m_{f}\right\rangle\left\langle F_{f}, m_{f}\left|, \quad \hat{P}_{e}=\sum_{m_{e}}\right| F_{e}, m_{e}\right\rangle\left\langle F_{e}, m_{e}\right|, \quad \hat{P}_{f}+\hat{P}_{e}=\mathbb{I} .
$$

Agora, a interação dos átomos com a radiação vai depender do operador de dipolo $\hat{\mathbf{d}}=e \hat{\mathbf{r}}$, que na base atômica é definido como $\hat{\mathbf{d}}=\mathbb{I} \hat{\mathbf{d}} \mathbb{I}=\left(\hat{P}_{f}+\hat{P}_{e}\right) \hat{\mathbf{d}}\left(\hat{P}_{f}+\hat{P}_{e}\right)$ de modo que $\hat{\mathbf{d}}$ seja impar por inversão espacial e o estados $\left|F_{f}, m_{f}\right\rangle$ e $\left|F_{e}, m_{e}\right\rangle$ podem ser escolhidos com paridade oposta, dando lugar a $\hat{P}_{f} \hat{\mathbf{d}} \hat{P}_{f}=\hat{P}_{e} \hat{\mathbf{d}} \hat{P}_{e}=0$, tal que o hamiltoniano de interação, levando em conta a aproximação de onda rotante (RWA), fica

$$
H_{\mathrm{Rad}}=\hbar\left[\Omega_{e f}\left(\mathbf{e}_{L} \cdot \mathbf{Q}_{e f}\right) e^{-i\left(\omega_{L} t+\phi_{L}\right)}+\Omega_{f e}\left(\mathbf{e}_{L} \cdot \mathbf{Q}_{f e}\right) e^{i\left(\omega_{L} t+\phi_{L}\right)}\right]
$$

onde $\mathbf{e}_{L}$ é a polarização do campo incidente, e $\omega_{L}$ e $\phi_{L}{ }^{5}$ correspondem à frequência

\footnotetext{
${ }^{5}$ Da mesma forma que no caso do sistema de três níveis, não consideramos a fase do campo $\phi_{L}$ como uma variável estocástica
} 
e à fase do laser incidente, respectivamente. O dipolo reduzido é definido como

$$
\hat{\mathbf{Q}}=\frac{\hat{\mathbf{d}}}{\left\langle F_{e}, m_{e}\|\hat{d}\| F_{f}, m_{f}\right\rangle} .
$$

tal que $\mathbf{Q}_{e f}=P_{e} \hat{\mathbf{Q}} P_{f}$ e $\mathbf{Q}_{f e}=\mathbf{Q}_{e f}^{\dagger}$, mediante o qual acoplamos estados com diferente energia e projeção de momento angular $m_{F}$. Finalmente a frequência de Rabi associada ao campo é

$$
\Omega_{e f}=\frac{E_{o}\left|\left\langle F_{e}, m_{e}|| \hat{d}|| F_{f}, m_{f}\right\rangle\right|}{\hbar},
$$

sendo $E_{o}$ a amplitude do campo incidente, que induz uma polarização determinada pelos níveis degenerados mediante o operador de dipolo reduzido $\hat{\mathbf{Q}}$.

\subsubsection{Interação com campo magnético}

Quando o campo incidente é linearmente polarizado e interage com um átomo de dois níveis degenerado, pode ser visto (na base circular) como dois campos com polarização $\sigma_{+}$ou $\sigma_{-}$, interagindo com o meio atômico, de modo que, ao manipular os níveis Zeeman com campos magnéticos, podemos induzir uma absorção preferencial para cada componente. Este fenômeno é conhecido como efeito Hanle, em que a interação com um campo magnético é descrito pelo hamiltoniano de dipolo magnético

$$
H_{B}=-\hat{\mu} \cdot \mathbf{B}
$$

sendo $\hat{\mu}$ o dipolo magnético dado por

$$
\hat{\mu}=\frac{\mu_{B}}{\hbar}\left[\hat{\mathbf{L}}+g_{e} \sum_{i} \hat{\mathbf{S}}_{e}^{i}-g_{N} \frac{m_{e}}{m_{N}} \hat{\mathbf{I}}\right],
$$

onde $\mu_{B}$ é o magnetón de Bohr, $g_{e}$ e $g_{N}$ são as constantes giromagnéticas do elétron e do núcleo respectivamente, $\hat{\mathbf{L}}$ é o momento angular orbital, $\hat{\mathbf{S}}_{e}^{i}$ o spin de cada elétron e $\hat{\mathbf{I}}$ é o spin núclear. O momento angular total do átomo é definido por $\hat{\mathbf{F}}=\hat{\mathbf{J}}+\hat{\mathbf{I}}$, sendo $\hat{\mathbf{J}}=\hat{\mathbf{L}}+\sum_{i} \hat{\mathbf{S}}_{e}^{i}$, cuja componente $z$ tem como base os estados $\left|F, m_{F}\right\rangle$ onde $\hbar m_{F}$ são seus autovalores.

Como $m_{e} \ll m_{N}$, podemos desprezar o último termo do momento dipolar (1.58), deixando só o momento angular $\hat{\mathbf{J}}$ acoplado ao campo magnético $H_{B}=-\left(\mu_{B} / \hbar\right) g_{J} \hat{\mathbf{J}}$. B . A projeção de $\hat{\mathbf{J}}$ sobre o momento angular total $\hat{\mathbf{F}}$ é determinado por $\hat{\mathbf{J}}=$ 
$(\hat{\mathbf{J}} \cdot \hat{\mathbf{F}}) \hat{\mathbf{F}} / F^{2}$. Se considerarmos o campo magnético como $\vec{B}=B \hat{\mathbf{z}}$, então obteremos o hamiltoniano $H_{B}$ em termos de $\hat{F}_{z}$ como é feito em [39], tal que

$$
H_{B}=-\left(\mu_{B} / \hbar\right) g_{F} B \hat{F}_{z}
$$

onde $g_{F}$ é o fator giromagnético para estados hiperfinos, dado por

$$
g_{F}=g_{J}\left(\frac{F(F+1)+J(J+1)-I(I+1)}{2 F(F+1)}\right) .
$$

Levando em conta que $\mu$ é par por reversão espacial $\left(\hat{P}_{e} \hat{\mu} \hat{P}_{f}=0\right)$, a quebra de degenerescência devido ao campo magnético é descrita pelo hamiltoniano

$$
H_{B}=\left[\gamma_{f} \hat{P}_{f}+\gamma_{e} \hat{P}_{e}\right] B \hat{F}_{z}
$$

onde $\gamma_{f}=\left(\mu_{B} g_{F(f)}\right) / \hbar$ e $\gamma_{e}=\mu_{B} g_{F(e)} / \hbar$. Dependendo do sinal do fator $\gamma_{e}$ e $\gamma_{f}$, a quebra de degenerescência pode ser sincronizada como se mostra na figura 1.9.a ou anti-sincronizada, como se mostra na figura 1.9.b. Então, o campo magnético cria
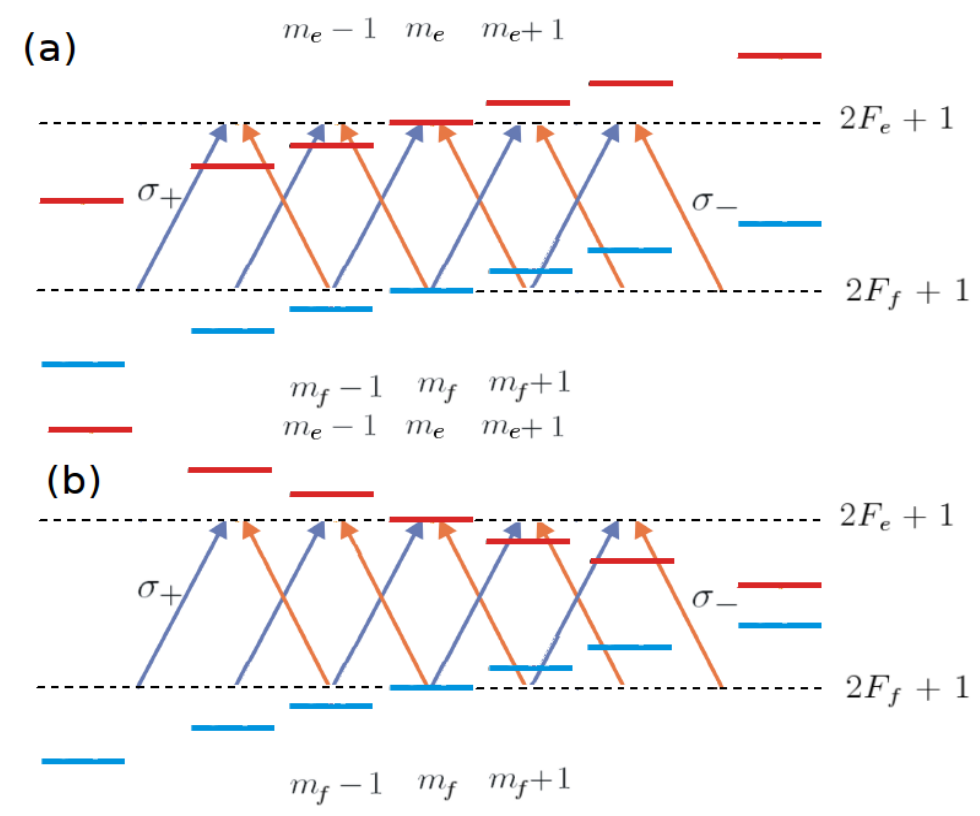

Figura 1.9: (a) Dessintonia sintonizada do laser com respeito dos níveis atômicos gerada pelo campo magnético quando $\gamma_{e}$ tem o mesmo signal que $\gamma_{f}$. (b) Dessintonia anti-sincronisada do laser, quando $\gamma_{e}$ tem o signal oposto a $\gamma_{f}$

uma dessintonia em frequência para os dois campos acoplados, tal que para ambos campos pode-se observar um perfil de absorção tipo EIT. 


\subsubsection{Contribuições dissipativas}

Nos sistemas degenerados, os canais de decaimento são um pouco mais complexos que no caso não degenerado (sistema de três níveis) ${ }^{6}$. Neste tipo de sistemas temos três possíveis canais pelos quais ocorre o decaimento do estado. Esses canais podem ser acessados por luz com polarização $\sigma_{+}, \sigma_{-}$e $\pi$. A taxa de decaimento neste tipo de sistemas é uma taxa reduzida em relação à taxa no caso não degenerado, ou seja

$$
\Gamma=\frac{\Gamma_{2 n i v}}{\left(2 F_{e}+1\right)} ; \quad \Gamma_{2 n i v}=\frac{\omega^{3}}{3 \pi \hbar c^{3}}\left|\left\langle E_{e}, F_{e}\|\hat{d}\| E_{f}, F_{f}\right\rangle\right|^{2}
$$

onde o fator $\Gamma_{2 n i v}$ é a taxa de decaimento de dois níveis não degenerados, de modo que $\Gamma$ é a fração de todas as transições possíveis entre níveis degenerados pelo número total de estados degenerados do nível excitado.

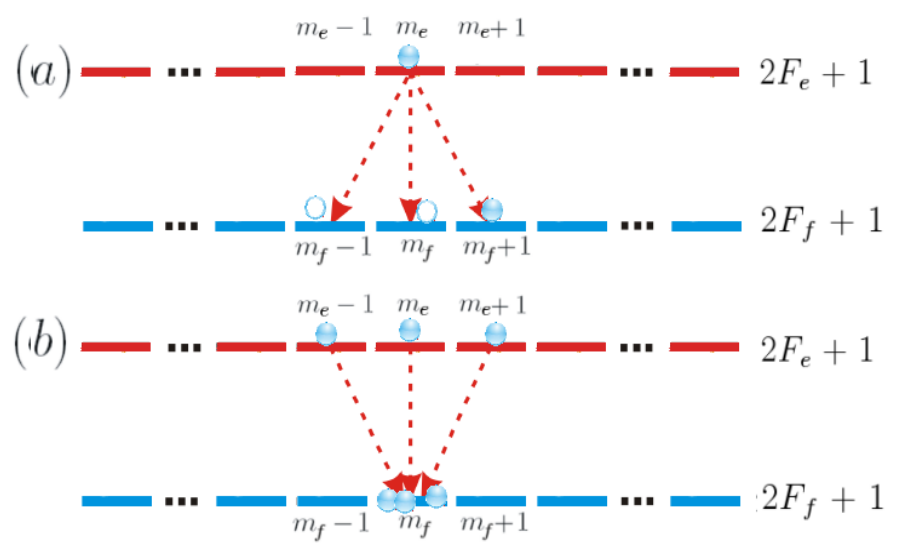

Figura 1.10: (a) Canais de relaxação do estado excitado $\left|F_{e}, m_{e}\right\rangle$. (b) Canais de população do estado fundamental $\left|F_{f}, m_{f}\right\rangle$

No caso do estado excitado $\left|F_{e}, m_{e}\right\rangle$, a dissipação na dinâmica da população por um desses canais depende unicamente da sua população, tal que

$$
\frac{d \rho_{m_{e} m_{e}^{\prime}}(t)}{d t}=-\Gamma \rho_{m_{e} m_{e}^{\prime}}(t)
$$

como se mostra na figura 1.10.a, em que vemos a representação do relaxamento da população do estado excitado por meio de um canal só $\left(\sigma_{+}, \sigma_{-}\right.$ou $\left.\pi\right)$. Isto mostra que não existe uma troca de coerências, ou seja, durante a evolução temporal, parte da probabilidade $\rho_{m_{e} m_{e}^{\prime}}(t)$ não depende de um fator $\rho_{m_{e} m_{f}^{\prime}}(t)$ e viceversa. Portanto,

\footnotetext{
${ }^{6} \mathrm{O}$ formalismo para calcular a taxa de decaimento pode ser encontrado em [30]
} 
para as coerências, temos que

$$
\frac{d \rho_{m_{f} m_{e}}(t)}{d t}=-\Gamma \rho_{m_{f} m_{e}}(t)
$$

da mesma forma que no caso de dois níveis não degenerados [30, 38, 39, 40]. Agora, no caso do estado fundamental $\left|F_{f}, m_{f}\right\rangle$, ele recebe populações à mesma taxa dos estados $\left|F_{e}, m_{e}+q\right\rangle$ onde $q=0, \pm 1$ como mostra a figura 1.10.b. A dinâmica da população do estado fundamental é dada por

$$
\frac{d \rho_{m_{f} m_{f}^{\prime}}(t)}{d t}=\left(2 F_{e}+1\right) \Gamma \sum_{q, m_{e}, m_{e}^{\prime}}\left\langle F_{e}, m_{e}\left|\hat{Q}_{q}^{\dagger} P_{e} \hat{\rho}(t) P_{e} \hat{Q}_{q}\right| F_{f}, m_{f}\right\rangle
$$

em que não temos apenas um ganho de população, mas também de coerências, reproduzindo o papel da taxa $\gamma_{21}$ no caso dos três níveis, que representava a decoerência entre os estados fundamentais.

O último termo dissipativo da expressão (1.51) é introduzido tipicamente de forma fenomenológica como um parâmetro para modelar as perdas pelo tamanho finito do feixe, tal que os átomos entram e saem da região de interação, no caso de átomos quentes em células de vapor. No entanto, no nosso sistema, esse efeito é mínimo, pois trabalhamos com átomos frios (embora um efeito similiar possa acontecer neste caso, devido a uma possível perda do controle de população). Especificamente, como a nuvem de átomos frios é preparada num estado de momento angular diferente, devemos bombear de maneira constante toda a população atômica para o estado $F_{f}$, acoplando-o com o estado $F_{e}$ para obter o espectro de EIT. Este controle de população pode não ser perfeito, de modo que existe uma pequena perda de população quando vamos acoplar os estados $F_{f} \rightarrow F_{e}$. A taxa com que esta pequena perda acontece pode ser modelada pela taxa $\gamma$ descrita pelo último termo $\gamma\left(\rho-\rho_{0}\right)$, onde $\rho_{0}=P_{f} / \sqrt{2 F_{f}+1}$. Este termo modela a variação de população $\left(\rho-\rho_{0}\right)$ num tempo $1 / \gamma$ para o sistema continuar numa dinâmica estacionária.

Analisamos os diferentes elementos que contribuem para a dinâmica das populações e para as coerências do sistema de dois níveis degenerados. Para solucionar essas equações acopladas, usamos uma representação no espaço de Liouville, onde $\rho \rightarrow y_{L}$, para obter uma expressão para a polarização da qual deduz-se a susceptibilidade $[29,28]$.

\subsection{Espaço de Liouville}

No formalismo de Liouville, associamos para o operador densidade $\hat{\rho}$ do espaço de Hilbert, o vetor $y_{L}$ no espaço de Liouville $\mathbb{L}$, de modo que todas as componentes da 
matriz densidade $\rho$, são agora as componentes de um arranjo vetorial no espaço de Liouville. Por outro lado, um operador $\mathcal{O}$, que atua sobre o operador densidade $\rho$, é representado no espaço de Liouville, dependendo do ordenamento dele relativo ao operador densidade, tal que

$$
\begin{aligned}
\mathcal{O} \rho & \rightarrow \mathcal{L}(\mathcal{O}) y \\
\rho \mathcal{O} & \rightarrow \mathcal{R}(\mathcal{O}) y
\end{aligned}
$$

em que $\mathcal{L}(\mathcal{O})$ e $\mathcal{R}(\mathcal{O})$ são operadores no espaço $\mathbb{L}$. Os operadores $\mathcal{L}(\mathcal{O})$ e $\mathcal{R}(\mathcal{O})$ representam o operador $\mathcal{O}$ atuando pela esquerda e pela direita de $\rho$, respectivamente. Especificamente no sistema de três níveis, para a matriz $w=\rho-\rho_{33} \mathbb{I}$ associamos o vetor $x_{3 n}$ tal que

$$
w=\left(\begin{array}{ccc}
w_{11} & \rho_{12} & \rho_{13} \\
\rho_{21} & w_{22} & \rho_{23} \\
\rho_{32} & \rho_{31} & 0
\end{array}\right) \Longrightarrow x_{3 n}=\left(w_{11}, w_{22}, \rho_{13}, \rho_{31}, \rho_{23}, \rho_{32}, \rho_{12}, \rho_{21}\right)
$$

de modo que a eq. (1.47), que descreve a dinâmica do sistema de três níveis, é reescrita no espaço de Liouville, como é feito em [29]

$$
\frac{d x_{3 n}}{d t}=\mathcal{L}(A) x_{3 n}+x_{0}
$$

onde a matriz A é definida na eq.(1.48) e o vetor $x_{0}=(-\Gamma,-\Gamma, 0,0,0,0,0,0)$. Por outro lado, para o sistema de dois níveis com degenerescências, com estados $\left|F_{f}, m_{f}\right\rangle$ e $\left|F_{e}, m_{e}\right\rangle$, temos a matriz de densidade

$$
\rho=\left(\begin{array}{cccccc}
\rho_{\left\{-F_{f},-F_{f}\right\}} & \ldots & \rho_{\left\{-F_{f}, F_{f}\right\}} & \rho_{\left\{-F_{f},-F_{e}\right\}} & \ldots & \rho_{\left\{-F_{f}, F_{e}\right\}} \\
\rho_{\left\{-F_{f}+1,-F_{f}\right\}} & \cdots & \rho_{\left\{-F_{f}+1, F_{f}\right\}} & \rho_{\left\{-F_{f}+1,-F_{e}\right\}} & \cdots & \rho_{\left\{-F_{f}+1, F_{e}\right\}} \\
\vdots & \vdots & \vdots & \vdots & \vdots & \vdots \\
\rho_{\left\{F_{f}-1,-F_{f}\right\}} & \cdots & \left\{F_{f}-1, F_{f}\right\} & \left\{F_{f}-1,-F_{e}\right\} & \cdots & \rho_{\left\{F_{f}-1, F_{e}\right\}} \\
\rho_{\left\{F_{f},-F_{f}\right\}} & \ldots & \left\{F_{f}, F_{f}\right\} & \left\{F_{f},-F_{e}\right\} & \cdots & \rho_{\left\{F_{f}, F_{e}\right\}} \\
\rho_{\left\{-F_{e},-F_{f}\right\}} & \cdots & \left\{-F_{e}, F_{f}\right\} & \left\{-F_{e},-F_{e}\right\} & \cdots & \rho_{\left\{-F_{e}, F_{e}\right\}} \\
\rho_{\left\{-F_{e}+1,-F_{f}\right\}} & \cdots & \left\{-F_{e}+1, F_{f}\right\} & \left\{-F_{e}+1,-F_{e}\right\} & \cdots & \rho_{\left\{-F_{e}+1, F_{e}\right\}} \\
\vdots & \vdots & \vdots & \vdots & \vdots & \vdots \\
\rho_{\left\{F_{e},-F_{f}\right\}} & \cdots & \left\{F_{e}, F_{f}\right\} & \left\{F_{e},-F_{e}\right\} & \cdots & \rho_{\left\{F_{e}, F_{e}\right\}}
\end{array}\right)
$$

para a qual associamos o vetor $y_{2 n}$ no espaço $\mathbb{L}$, dado por

$$
y_{2 n}=\left(\rho_{\left\{-F_{f},-F_{f}\right\}}, \ldots, \rho_{\left\{-F_{f}, F_{e}\right\}}, \rho_{\left\{-F_{f}+1,-F_{f}\right\}}, \ldots, \rho_{\left\{-F_{f}+1, F_{e}\right\}}, \ldots, \ldots, \rho_{\left\{F_{e},-F_{f}\right\}}, \ldots, \rho_{\left\{F_{e}, F_{e}\right\}}\right)^{T}
$$

Usando os operadores das eqs. (1.66) e (1.67), podemos reescrever a equação dinâmica de $\rho$, no espaço $\mathbb{L}$, como é feito em $[29,28]$, de modo que

$$
\frac{d y_{2 n}}{d t}=e^{-i N \omega_{L} t} \mathcal{A}_{2 \text { niv }} e^{i N \omega_{L} t} y_{2 n}+\gamma y_{0}
$$


sendo

$$
\begin{aligned}
N & =\mathcal{L}\left(P_{e}\right)-\mathcal{R}\left(P_{e}\right), \\
\mathcal{A}_{2 \text { niv }} & =-\frac{i}{\hbar}\left[\mathcal{L}\left(H_{0}+H_{B}+H_{\mathrm{Rad}}\right)-\mathcal{R}\left(H_{0}+H_{B}+H_{\mathrm{Rad}}\right)\right] \\
& -\frac{\Gamma}{2}\left[\mathcal{L}\left(P_{e}\right)+\mathcal{R}\left(P_{e}\right)\right]-\gamma \mathbb{I} \\
& +\Gamma\left(2 F_{e}+1\right) \sum_{q=-1,0,1} \mathcal{L}\left(Q_{f e}^{q}\right) \mathcal{R}\left(Q_{f e}^{q}\right)
\end{aligned}
$$

e $\rho_{0} \rightarrow y_{0}$, onde $\mathbb{I}$ é a matriz indentidade no espaço $\mathbb{L}$. Fazendo uma tranformação das variáveis lentas, definimos o vetor $x_{2 n}=\exp \left(i N \omega_{L} t\right) y_{2 n}$ como foi feito na eq. (1.27), cuja dinâmica é dada por

$$
\frac{d x_{2 n}}{d t}=\left(i N \omega_{L}+\mathcal{A}_{2 \text { niv }}\right) x_{2 n}+\gamma y_{0} .
$$

A partir da solução no regime estacionário, ou seja, em cada um dos sistemas achamos a solução quando $\frac{d x_{3 n}}{d t}=0$ e $\frac{d x_{2 n}}{d t}=0$, e levando em conta que $\operatorname{Tr}(\mathcal{O} \rho)=$ $u^{\dagger} \mathcal{L}(\mathcal{O}) y$, a polarização atômica nos dois sistemas é dada por

$$
\mathbf{P}_{2 n}=u^{\dagger} \mathcal{L}\left(\mathcal{D}_{f e}\right) x_{2 n}, \mathbf{P}_{3 n}=u^{\dagger} \mathcal{L}\left(\mathcal{D}_{13}\right) x_{3 n}
$$

onde $u$ é o vetor cujas componentes de população são unitárias e as coerências são nulas. A partir da solução para a polarização de cada um dos sistemas, é possível determinar uma expressão para a susceptibilidade $\chi$. 


\section{CAPítUlo 2}

\section{Modelo de Difusão de fase para o ruído e a correlação dos campos eletromágneticos, em sistema de 3 níveis}

No capítulo anterior estudamos a interação da luz com o meio atômico em condições de EIT e Hanle-EIT, considerando apenas a absorção do valor médio dos campos electromagnéticos. Neste capítulo vamos analisar o que acontece com as flutuações desses campos na presença de átomos. O modelo que tratará o ruído dos campos, é o modelo de Difusão de Fase, trabalhado anteriomente em experimentos do laboratório [33].

Classicamente, o campo elétrico possui uma amplitude e uma fase muito bem definidas, tal que

$$
\mathbf{E}(\mathbf{r}, t)=\mathcal{E}_{0} e^{i(\mathbf{k} \cdot \mathbf{r}-\omega t)} \hat{\mathbf{e}}+\mathcal{E}_{0}^{*} e^{-i(\mathbf{k} \cdot \mathbf{r}-\omega t)} \hat{\mathbf{e}} ; \mathcal{E}_{0}=\left|\mathcal{E}_{0}\right| e^{i \phi},
$$

onde $\mathcal{E}_{0}$ é a amplitude do campo e $\mathbf{k} \cdot \mathbf{r}-\omega t+\phi$ corresponde à fase do campo. Do ponto de vista clássico, estas variáveis são estatisticamente definidas com certeza, ou seja, não apresentam flutuações, como é mostrado na figura 2.1.a. Dado que a amplitude do campo em geral é complexa, ou seja, $\mathcal{E}_{0}=-\frac{E_{P}}{2}+i \frac{E_{Q}}{2}$, então o campo pode ser escrito como

$$
\mathbf{E}(\mathbf{r}, t)=-E_{P} \cos (\mathbf{k} \cdot \mathbf{r}-\omega t) \hat{\mathbf{e}}-E_{Q} \sin (\mathbf{k} \cdot \mathbf{r}-\omega t) \hat{\mathbf{e}}
$$

onde $E_{P}$ e $E_{Q}$ são as quadraturas de amplitude e de fase do campo, respectivamente. Numa representação de Fresnel (diagrama de fasor), mostramos na figura 2.1.b a amplitude do campo $\mathcal{E}_{0}$ como um vetor muito bem definido, cuja parte real corresponde à quadratura $E_{P}$ e a parte imaginária corresponde à quadratura $E_{Q}$. 


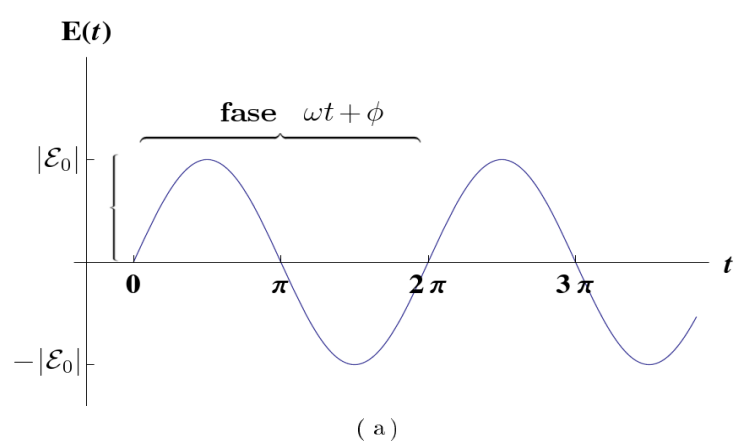

( a )

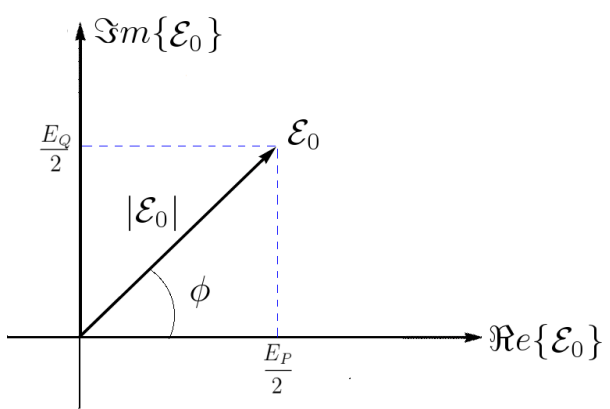

(b)

Figura 2.1: (a) Representação da amplitude do campo elétrico $E(t)$ no tempo, para uma posição $\mathbf{r}=0$. (b) Representação da amplitude $\mathcal{E}_{0}$ em fasores.

Entretanto, mediante o formalismo quântico dos campos electromagnéticos, é possivel mostrar que as quadraturas, num modo do campo específico, satisfazem uma relação de incerteza tipo Heisenberg [14, 38, 40]

$$
\Delta E_{Q} \Delta E_{P} \geq \Delta^{2} E_{\text {Shot }}
$$

em que $\Delta^{2} E_{\text {Shot }}$ corresponde ao ao mínimo de incerteza das quadraturas denominado Standard Quantum Limit (SQL). Quando os campos possuem este mínimo de incerteza nas suas quadraturas, ou seja, $\Delta E_{Q}=\Delta E_{P}=\sqrt{\Delta^{2} E_{\text {Shot }}}$, seu estado é coerente. O limite SQL pode ser acessado medindo o Shot-noise de um estado coerente (ver circulo de linha vermelha na figura 2.2.b). No entanto o campo pode apresentar excesso de ruído em alguma das suas quadraturas, como se mostra na figura 2.2.a, em que na representação temporal do campo podemos observar uma
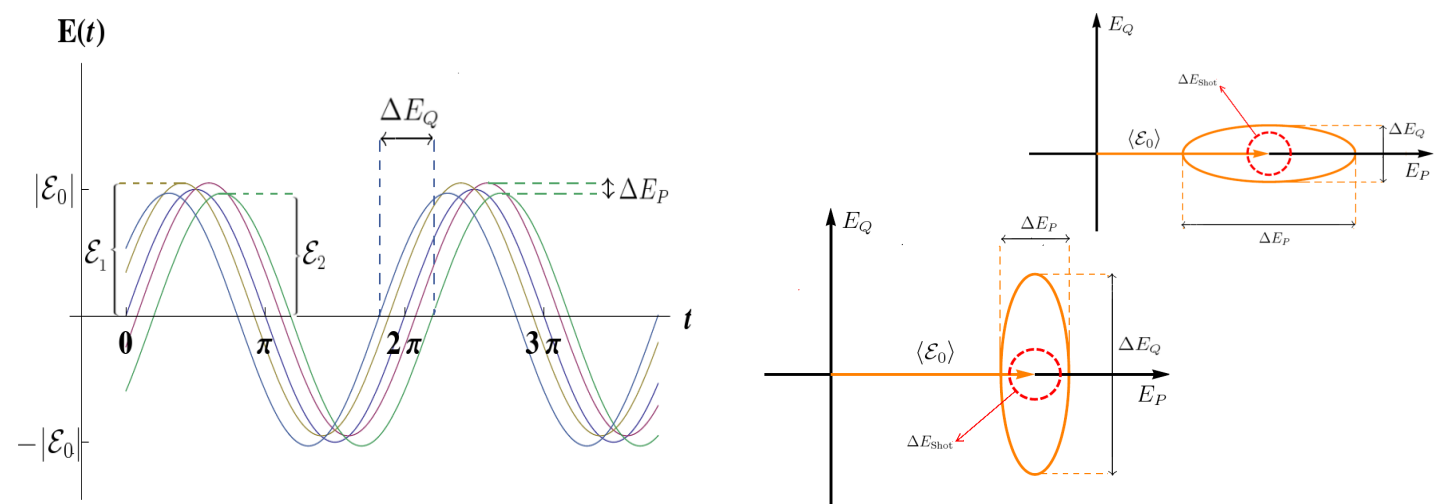

Figura 2.2: (a) Representação da amplitude do campo elétrico $E(t)$ no tempo, com flutuações de fase, para uma posição $\mathbf{r}=0$. (b) Representação de Fresnel do valor esperado da amplitude do campo $\left\langle\mathcal{E}_{0}\right\rangle$. A Linha vermelha mostra o limite mínimo quântico de ruído (Shot-noise). A Linha laranja representa o estado do campo com excesso de ruído de fase (figura embaixo) e amplitude (figura acima)

variação da defasagem do campo no tempo, representando assim, a incerteza na fase. 
Na figura 2.2.b, diagrama de Fresnel, mostramos o caso no qual aparece o excesso de ruído de fase e excesso de ruído de amplitude.

O ruído dos campos após a interação com o meio atômico é transformado e pode ser modelado com base no modelo de Difusão de Fase, que leva em conta que os campos incidentes possuem um excesso nas flutuações de fase, de modo que após interagir com os átomos, transformam o ruído de fase em ruído de amplitude, como mostra a figura 2.3. Isto foi proposto por Yabuzaki et al em [41]. Este ruído de amplitude pode ser analisado nas suas diferentes componentes de frequência, calculando o espectro de potência a partir do teorema de Wiener-Khintchine. Isto permite a comparação direta com nosso esquema de medida que é feito no dominio das frequências.

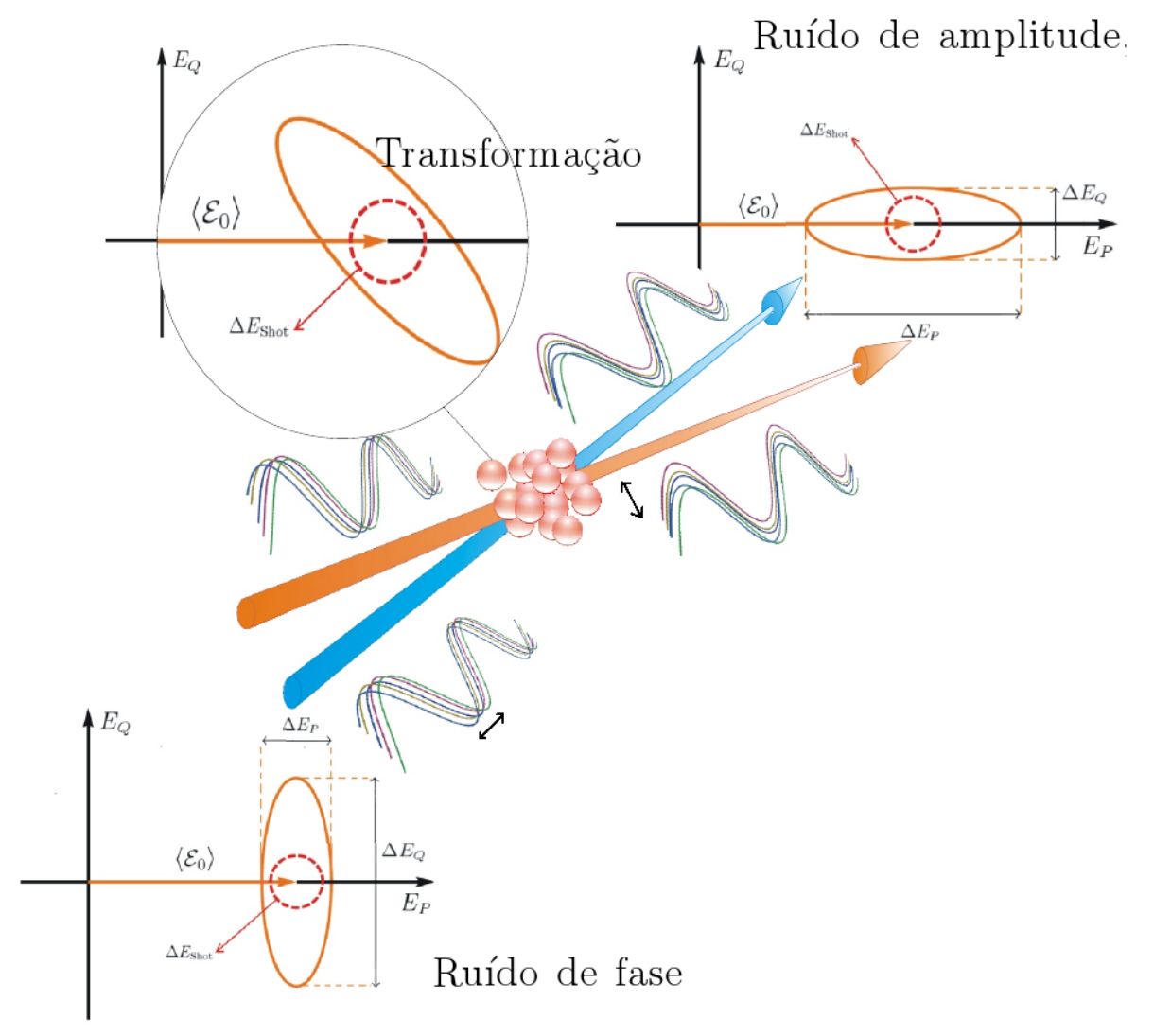

Figura 2.3: Esquema do modelo de Difusão de fase. Incialmente consideramos os campos incidentes como campos com exceso de ruído de fase, posteriormente eles interagem com os átomos, encarregados de transformar o exceso de ruído de fase. Finalmente os campos transmitidos possuem um excesso de ruído na quadratura de amplitude.

O modelo que vamos apresentar pode ser usado no sistema de três níveis e no sistema de dois níveis degenerados. Vamos estudar o sistema de três níveis, 
que pode ser visto como um caso simples, e pode ser extendido para o sistema de dois niveis (sem considerar Hanle-EIT), gerando resultados comparáveis aos dos resultados experimentais.

Para começar, consideraremos dois campos clássicos intensos, representados por dois lasers, descritos por

$$
\mathbf{E}_{i}(t)=\mathcal{E}_{i} e^{i\left(\omega_{i} t+\phi_{i}(t)\right)} \mathbf{e}_{i}
$$

onde $i=1,2$ indica cada um dos campos, $\mathbf{e}_{i}$ é a polarização de cada campo, a variável $\mathcal{E}_{i}$ é uma amplitude constante e complexa (relacionada como valor médio da intesidade do campo) e $\omega_{i}$ é a frequência de cada um dos lasers. A evolução temporal das fases $\phi_{1}(t)$ e $\phi_{2}(t)$ é descrita como processos independentes de Wiener, para variáveis aleatórias correlacionadas, tal que

$$
\begin{aligned}
\left\langle d \phi_{i}\right\rangle & =0, \\
\left\langle d \phi_{i} d \phi_{k}\right\rangle & =2 \sqrt{\gamma_{i} \gamma_{k}} d t, \\
\left\langle d \phi_{i}^{n}\right\rangle & =0, \quad n>2,
\end{aligned}
$$

onde $2 \gamma_{1}$ e $2 \gamma_{2}$ correpondem à largura espectral do perfil lorentziano de cada um dos campos. No caso do laser de diodo, usado normalmente nas experiências do laboratório, vê-se um excesso do ruído de fase, modelado justamente por $\left\langle d \phi_{i}^{2}\right\rangle=$ $2 b_{i} d t$, de modo que os átomos transformam esse excesso de ruído em ruído nas amplitudes dos campos.

Para modelar a resposta atômica consideramos que o campo de saída, após a interação com os átomos, é composto pelos campos incidentes e o campo emitido pela polarização atômica, tal que

$$
\mathbf{E}_{S}(t)=\mathbf{E}_{1}(t)+\mathbf{E}_{2}(t)+i \kappa \mathbf{P}(t),
$$

onde $\kappa$ é só uma constante de proporcionalidade da polarização $\mathbf{P}(t)$ do meio atômico, definida anteriormente na eq. (1.36). Esta é reescrita em termos das variáves rapidas no tempo ${ }^{1}$ como

$$
\mathbf{P}(t)=\left[\mathbf{p}_{1}\left(t, \omega_{1}\right) e^{i\left(\omega_{1} t+\phi_{1}\right)}+\mathbf{p}_{2}\left(t, \omega_{2}\right) e^{i\left(\omega_{2} t+\phi_{2}\right)}\right],
$$

onde $\mathbf{p}_{1}\left(t, \omega_{1}\right)$ e $\mathbf{p}_{2}\left(t, \omega_{2}\right)$ são as polarizações induzidas pelos campos $\mathbf{E}_{1}(t)$ e $\mathbf{E}_{2}(t)$, respectivamente. Dado que nas experiências feitas no laboratório usamos átomos frios, isso significa que podemos considerar a velocidade dos átomos nula com respeito ao referencial do laboratório. Isto implica em não precisarmos levar em conta o perfil Doppler, simplificando assim a expressão para a polarização da eq. (2.9). Caso

\footnotetext{
${ }^{1}$ Deixamos as constante de probabilidade definidas dentro da polarização, dentro da constante $\kappa$
} 
contrário, precisaríamos integrar essa polarização numa distribuição de velocidades como é feito em [29,33].

O modelo pode ser descrito para o caso em que temos dois campos transmitidos linearmente polarizados $\hat{\mathbf{e}}_{q}=1,2$ em condição de EIT [33], ou para o caso de termos dois campos com polarizações circulares opostas $\hat{\mathbf{q}}=\sigma_{+}, \sigma_{-}$[28]. Vamos considerar o primeiro caso, em que a intensidade transmitida num dos feixes é definida como a projeção deste sobre o campo total de saída, ou seja,

$$
I_{q}(t)=\left|\mathbf{E}_{S}(t) \cdot \mathbf{e}_{q}\right|^{2} .
$$

Para determinar o espectro de potência a partir da intensidade $I_{q}(t)$, usamos o teorema de Wiener-Khintchine [42], que relaciona o espectro do campo $S_{q q^{\prime}}(\omega)$ mediante a transformada de Fourier da função de auto-correlação da intensidade $I_{q}(t)$, tal que

$$
S_{q q^{\prime}}(\omega) \equiv \int_{-\infty}^{\infty}\left[\left\langle I_{q}(t+\tau) I_{q^{\prime}}(t)\right\rangle-\left\langle I_{q}(t+\tau)\right\rangle\left\langle I_{q^{\prime}}(t)\right\rangle\right] e^{i \omega \tau} d \tau \text {. }
$$

Substituindo a expressão do campo de saída (2.8) na eq.(2.10), obtemos a intensidade

$$
\begin{aligned}
I_{q}(t) & =\left|\left[\mathbf{E}_{q}(t)+i \kappa \mathbf{p}_{q}\left(t, \omega_{q}\right) e^{i\left(\omega_{1} t+\phi_{1}\right)}\right] \cdot \mathbf{e}_{q}\right|^{2} \\
& =I_{q}^{0}+i \kappa \mathcal{E}_{q} p_{q}\left(t, \omega_{q}\right)-i \kappa \mathcal{E}_{q} p_{q}^{*}\left(t, \omega_{q}\right)+\mathcal{O}\left(\kappa^{2}\right),
\end{aligned}
$$

onde $I_{q}^{0}=\mathcal{E}_{q}^{2}$. Dada esta expressão para a intensidade, podemos calcular o produto $I_{q}(t+\tau) I_{q^{\prime}}(t)$ de modo que

$$
\begin{aligned}
I_{q}(t+\tau) I_{q^{\prime}}(t)= & {\left[I_{q}^{0}+i \kappa \mathcal{E}_{q}\left(p_{q}\left(t+\tau, \omega_{q}\right)-p_{q}^{*}\left(t+\tau, \omega_{q}\right)\right)\right] } \\
& \times\left[I_{q^{\prime}}^{0}+i \kappa \mathcal{E}_{q^{\prime}}\left(p_{q^{\prime}}\left(t, \omega_{q^{\prime}}\right)-p_{q^{\prime}}^{*}\left(t, \omega_{q^{\prime}}\right)\right)\right] \\
= & I_{q}^{0} I_{q^{\prime}}^{0}+i \kappa I_{q}^{0} \mathcal{E}_{q^{\prime}}\left[p_{q^{\prime}}\left(t, \omega_{q^{\prime}}\right)-p_{q^{\prime}}^{*}\left(t, \omega_{q^{\prime}}\right)\right] \\
& +i \kappa I_{q^{\prime}}^{0} \mathcal{E}_{q}\left[p_{q}\left(t+\tau, \omega_{q}\right)-p_{q}^{*}\left(t+\tau, \omega_{q}\right)\right] \\
& -\kappa^{2} \mathcal{E}_{q} \mathcal{E}_{q^{\prime}}\left[p_{q}\left(t+\tau, \omega_{q}\right)-p_{q}^{*}\left(t+\tau, \omega_{q}\right)\right] \times\left[p_{q^{\prime}}\left(t, \omega_{q^{\prime}}\right)-p_{q^{\prime}}^{*}\left(t, \omega_{q^{\prime}}\right)\right] .
\end{aligned}
$$

A partir desta equação e levando em conta que $\left\langle p_{q}\left(t+\tau, \omega_{q}\right)\right\rangle=\left\langle p_{q}\left(t, \omega_{q}\right)\right\rangle$ no regime estacionário, obtemos uma expressão para o espectro $S_{q q^{\prime}}(\omega)$ dada por

$$
\begin{array}{r}
S_{q q^{\prime}}(\omega)=-\kappa^{2} \mathcal{E}_{q} \mathcal{E}_{q^{\prime}} \int_{-\infty}^{\infty} d \tau e^{i \omega \tau}\left\{\left[\left\langle p_{q}\left(t+\tau, \omega_{q}\right) p_{q^{\prime}}\left(t, \omega_{q^{\prime}}\right)\right\rangle-\left\langle p_{q}\left(t+\tau, \omega_{q}\right)\right\rangle\left\langle p_{q^{\prime}}\left(t, \omega_{q^{\prime}}\right)\right\rangle\right]\right. \\
\left.-\left[\left\langle p_{q}\left(t+\tau, \omega_{q}\right) p_{q^{\prime}}^{*}\left(t, \omega_{q^{\prime}}\right)\right\rangle-\left\langle p_{q}\left(t+\tau, \omega_{q}\right)\right\rangle\left\langle p_{q^{\prime}}^{*}\left(t, \omega_{q^{\prime}}\right)\right\rangle\right]+c . c\right\} .
\end{array}
$$

Cada uma das polarizações $p_{q}$ está relacionada diretamente com as coerências atômicas $\rho_{q 3}$ sendo $q=1,2$, tratadas no capítulo anterior. Portanto, resolvendo as equações 
dinâmicas (1.20)-(1.25) ${ }^{2}$ das populações, e as coerências, obtemos a solução de $p_{q}$. Para achar a solução, vamos usar o espaço de Liouville descrito na seção 1.6, na qual definimos o arranjo de variáveis rapidas $y=\left(w_{11}, w_{22}, \rho_{13}, \rho_{31}, \rho_{23}, \rho_{32}, \rho_{12}, \rho_{21}\right)$ e o arranjo das variáveis lentas $x=\left(w_{11}, w_{22}, p_{1}, p_{1}^{*}, p_{2}, p_{2}^{*} p_{3}, p_{3}^{*}\right)^{3}$. A transformação descrita em (1.26), pode ser vista como

$$
\begin{aligned}
& y=e^{-i N_{1}\left(\omega_{1} t+\phi_{1}(t)\right)} e^{-i N_{2}\left(\omega_{2} t+\phi_{2}(t)\right)} x, \\
& x=e^{i N_{1}\left(\omega_{1} t+\phi_{1}(t)\right)} e^{i N_{2}\left(\omega_{2} t+\phi_{2}(t)\right)} y,
\end{aligned}
$$

onde temos que $\phi_{1}(t)$ e $\phi_{2}(t)$ são as fases estocásticas dos campos. As matrizes $N_{1}$ e $N_{2}$ são definidas por

$$
N_{1}=\left[\begin{array}{ccccccccc}
0 & 0 & 0 & 0 & 0 & 0 & 0 & 0 & 0 \\
0 & 0 & 0 & 0 & 0 & 0 & 0 & 0 & 0 \\
0 & 0 & 1 & 0 & 0 & 0 & 0 & 0 & 0 \\
0 & 0 & 0 & -1 & 0 & 0 & 0 & 0 & 0 \\
0 & 0 & 0 & 0 & 0 & 0 & 0 & 0 & 0 \\
0 & 0 & 0 & 0 & 0 & 0 & 0 & 0 & 0 \\
0 & 0 & 0 & 0 & 0 & 0 & 0 & 1 & 0 \\
0 & 0 & 0 & 0 & 0 & 0 & 0 & 0 & -1
\end{array}\right], N_{2}=\left[\begin{array}{ccccccccc}
0 & 0 & 0 & 0 & 0 & 0 & 0 & 0 & 0 \\
0 & 0 & 0 & 0 & 0 & 0 & 0 & 0 & 0 \\
0 & 0 & 0 & 0 & 0 & 0 & 0 & 0 & 0 \\
0 & 0 & 0 & 0 & 0 & 0 & 0 & 0 & 0 \\
0 & 0 & 0 & 0 & 1 & 0 & 0 & 0 & 0 \\
0 & 0 & 0 & 0 & 0 & -1 & 0 & 0 & 0 \\
0 & 0 & 0 & 0 & 0 & 0 & 0 & -1 & 0 \\
0 & 0 & 0 & 0 & 0 & 0 & 0 & 0 & 1
\end{array}\right],
$$

onde $\left[N_{1}, N_{2}\right]=0$, de modo que as eqs. (1.20)-(1.25) podem ser reescritas, em função dos vetores $y$ e $x$, da seguinte forma:

$$
\frac{d y}{d t}=e^{-i N_{1}\left(\omega_{1} t+\phi_{1}(t)\right)} e^{-i N_{2}\left(\omega_{2} t+\phi_{2}(t)\right)} \mathcal{A} x+y_{0},
$$

onde $\mathcal{A}=A-i N_{1} \omega_{1}-i N_{2} \omega_{2}$, sendo $A$ a matriz definida em (1.48), que comuta com as matrizes $N_{1}$ e $N_{2}$. No entanto, estamos interesados na dinâmica do arranjo $x$, que contem as polarizações $p_{q}$. Para isso usamos a transformação (2.16),levando em conta o caráter estocástico das fases que obrigam a calcular $d x$ mediante a fórmula de Ito [42] até segunda ordem, dada por

$$
\begin{aligned}
d\left[f\left(\phi_{1}, \phi_{2}, t\right)\right]=\frac{\partial f}{\partial t} d t+\frac{\partial f}{\partial \phi_{1}} d \phi_{1} & +\frac{\partial f}{\partial \phi_{2}} d \phi_{2}+\frac{1}{2} \frac{\partial^{2} f}{\partial \phi_{1}^{2}} d \phi_{1}^{2} \\
& +\frac{1}{2} \frac{\partial^{2} f}{\partial \phi_{2}^{2}} d \phi_{2}^{2}+\frac{\partial^{2} f}{\partial \phi_{1} \partial \phi_{2}} d \phi_{1} d \phi_{2},
\end{aligned}
$$

\footnotetext{
${ }^{2} \mathrm{~A}$ única diferença que devemos levar em conta nas eqs.(1.20)-(1.25), é que $\omega_{p} \rightarrow \omega_{1}$ e $\omega_{c} \rightarrow \omega_{2}$, $\phi_{p} \rightarrow \phi_{1}$ e $\phi_{c} \rightarrow \phi_{2}$

${ }^{3}$ Consideramos $p_{q}=\tilde{\rho}_{q 3}$ sendo $\tilde{\rho}_{q 3}$ as vairiáveis lentas definidas na seção 1.4
} 
de modo que a equação para $d x$ é determinada por

$$
\begin{aligned}
d x= & {\left[i N_{1}\left(\omega_{1} d t+d \phi_{1}\right)-\frac{N_{1}^{2} d \phi_{1}^{2}}{2}+i N_{2}\left(\omega_{2} d t+d \phi_{2}\right)-\frac{N_{2}^{2} d \phi_{2}^{2}}{2}-N_{1} N_{2} d \phi_{1} d \phi_{2}\right] x } \\
& +\mathcal{A} x d t+y_{0} d t
\end{aligned}
$$

onde usamos o fato de que $e^{-i N_{j}\left(\omega_{j} t+\phi_{j}\right)} y_{0}=y_{0}$. Agora calculamos o valor médio de $x$, considerando as propiedades $(2.5)$ e (2.6) das fases, e o fato de $x$ não ser diretamente correlacionado com as fases, o que implica em $\left\langle d \phi_{j} x(t)\right\rangle=\left\langle d \phi_{j}\right\rangle\langle x(t)\rangle$ e portanto

$$
d\langle x\rangle=\underbrace{\left[i N_{1} \omega_{1}-\gamma_{1} N_{1}^{2}+i N_{2} \omega_{2}-\gamma_{2} N_{2}^{2}-2 N_{1} N_{2} \gamma_{12}+\mathcal{A}\right]}_{-A_{T}}\langle x\rangle d t+y_{0} d t,
$$

tal que

$$
\frac{d\langle x\rangle}{d t}=-A_{T}\langle x\rangle+y_{0}
$$

Podemos observar que esta equação é muito parecida com a eq. (1.47), na qual não foi considerado o caráter estocástico das fases dos campos, como foi feito nesse caso. No regime estacionário, obtemos

$$
A_{T}\langle x\rangle_{\mathbf{e}}=y_{0}
$$

onde a matriz $A_{T}$ e dada por

$$
A_{T}=\left[\gamma_{1} N_{1}^{2}+\gamma_{2} N_{2}^{2}+2 N_{1} N_{2} \gamma_{12}\right]-i\left[N_{1} \omega_{1}+N_{2} \omega_{2}\right]-\mathcal{A}
$$

\subsection{Matriz de Covariância}

Dado que o espectro do campo eletromagnético depende dos termos $\left\langle p_{q}(t+\tau) p_{q^{\prime}}(t)\right\rangle$, então podem ser vistos como elementos de matriz $\left\langle C_{q q^{\prime}}(t+\tau)\right\rangle$, da matriz de correlação de segunda ordem $C(t+\tau)$. A partir dela pode-se derivar a matriz de covariância com a qual se obtém os espectos dos campos. Começaremos com a definição da matriz de correlação

$$
C(t+\tau, t)=x(t+\tau) x^{\dagger}(t),
$$

tal que em representação matricial temos

$$
C(t+\tau, t)=\left(\begin{array}{ccccc}
w_{11}(t+\tau) w_{11}(t) & w_{11}(t+\tau) w_{22}(t) & w_{11}(t+\tau) p_{1}(t) & \cdots & w_{11}(t+\tau) p_{3}(t) \\
w_{22}(t+\tau) w_{11}(t) & w_{22}(t+\tau) w_{22}(t) & w_{22}(t+\tau) p_{1}(t) & \cdots & w_{22}(t+\tau) p_{3}(t) \\
p_{1}(t+\tau) w_{11}(t) & p_{1}(t+\tau) w_{22}(t) & p_{1}(t+\tau) p_{1}(t) & \cdots & p_{1}(t+\tau) p_{3}(t) \\
\vdots & \vdots & \vdots & \vdots & \vdots \\
p_{3}^{*}(t+\tau) w_{11}(t) & p_{3}^{*}(t+\tau) w_{22}(t) & p_{3}^{*}(t+\tau) p_{1}(t) & \cdots & p_{3}^{*}(t+\tau) p_{3}(t)
\end{array}\right)
$$


A partir desta matriz de correlação, a matriz de covariância é definida por

$$
\mathfrak{C}(t+\tau, t)=C(t+\tau, t)-x(t+\tau)\left\langle x^{\dagger}(t)\right\rangle .
$$

Vamos considerar a dinâmica da matriz $C(t)$, para o caso em $\tau=0$, com a qual podemos contruir a dinâmica para a matriz de covariância. Para isso retomamos a tranformação (2.16) tal que

$$
C(t, t)=e^{i N_{1}\left(\omega_{1} t+\phi_{1}(t)\right)} e^{i N_{2}\left(\omega_{2} t+\phi_{2}(t)\right)} y(t) y^{\dagger}(t) e^{-i N_{1}\left(\omega_{1} t+\phi_{1}(t)\right)} e^{-i N_{2}\left(\omega_{2} t+\phi_{2}(t)\right)} .
$$

Usando a dinâmica de $y(t)$ da eq. (2.18), podemos calcular

$$
\begin{aligned}
d C(t)= & {\left[i N_{1}\left(\omega_{1} d t+d \phi_{1}\right)-\frac{N_{1}^{2} d \phi_{1}^{2}}{2}+i N_{2}\left(\omega_{2} d t+d \phi_{2}\right)\right.} \\
& \left.-\frac{N_{2}^{2} d \phi_{2}^{2}}{2}-N_{1} N_{2} d \phi_{1} d \phi_{2}+\mathcal{A} d t\right] x(t) x^{\dagger}(t)+y_{0} x^{\dagger}(t) d t \\
& +x(t) x^{\dagger}(t)\left[-i N_{1}\left(\omega_{1} d t+d \phi_{1}\right)-\frac{N_{1}^{2} d \phi_{1}^{2}}{2}-i N_{2}\left(\omega_{2} d t+d \phi_{2}\right)\right. \\
& \left.-\frac{N_{2}^{2} d \phi_{2}^{2}}{2}-N_{1} N_{2} d \phi_{1} d \phi_{2}+\mathcal{A}^{\dagger} d t\right]+x(t) y_{0} d t \\
& +N_{1} x(t) x^{\dagger}(t) N_{1} d \phi_{1}^{2}+N_{2} x(t) x^{\dagger}(t) N_{2} d \phi_{2}^{2} \\
& +N_{1} x(t) x^{\dagger}(t) N_{2} d \phi_{1} d \phi_{2}+N_{2} x(t) x^{\dagger}(t) N_{1} d \phi_{1} d \phi_{2} .
\end{aligned}
$$

Calculando a média desta expressão e a solução de $\langle x(t)\rangle$ da eq.(2.22), obtemos

$$
\begin{aligned}
d\langle C(t)\rangle= & -A_{T}\langle C(t)\rangle+\langle C(t)\rangle A_{T}^{\dagger}+y_{0}\left\langle x^{\dagger}(t)\right\rangle d t+\langle x(t)\rangle y_{0} d t \\
& +2 \gamma_{1} N_{1}\langle C(t)\rangle N_{1}+2 \gamma_{2} N_{2}\langle C(t)\rangle N_{2} \\
& +2 \gamma_{12} N_{1}\langle C(t)\rangle N_{2}+2 \gamma_{12} N_{2}\langle C(t)\rangle N_{1},
\end{aligned}
$$

de modo que numa situação estacionária onde $\frac{d\langle C(t)\rangle}{d t}=0$, podemos calcular a solução neste regime, para a matriz de covariância, usando a definição (2.27), levando em conta que $A_{T}\langle x(t)\rangle=y_{0}$, tal que

$$
\begin{aligned}
& A_{T}\langle\mathfrak{C}(t)\rangle-\langle\mathfrak{C}(t)\rangle A_{T}^{\dagger}+2 \gamma_{1} N_{1}\langle\mathfrak{C}(t)\rangle N_{1}+2 \gamma_{2} N_{2}\langle\mathfrak{C}(t)\rangle N_{2} \\
& +2 \gamma_{12} N_{1}\langle\mathfrak{C}(t)\rangle N_{2}+2 \gamma_{12} N_{2}\langle\mathfrak{C}(t)\rangle N_{1} \\
& =2 \gamma_{1} N_{1}\langle x(t+\tau)\rangle\left\langle x^{\dagger}(t)\right\rangle N_{1}+2 \gamma_{2} N_{2}\langle x(t+\tau)\rangle\left\langle x^{\dagger}(t)\right\rangle N_{2} \\
& +2 \gamma_{12} N_{1}\langle x(t+\tau)\rangle\left\langle x^{\dagger}(t)\right\rangle N_{2}+2 \gamma_{12} N_{2}\langle x(t+\tau)\rangle\left\langle x^{\dagger}(t)\right\rangle N_{1} .
\end{aligned}
$$

Agora, consideremos o caso quando $\tau \neq 0$, de modo que a evolução temporal (com respeito a $\tau$ ) da matriz de covariância e dada por

$$
\frac{d\langle\mathfrak{C}(t+\tau, t)\rangle}{d \tau} d \tau=\frac{d\langle C(t+\tau, t)\rangle}{d \tau} d \tau-\frac{d\langle x(t+\tau)\rangle}{d \tau} d \tau\left\langle x^{\dagger}(t)\right\rangle .
$$


Usando o teorema da regressão [43], mostra-se que, se a dinâmica de um elemento da matriz $M(t)$ é dada por

$$
\frac{d M_{q}(t)}{d t}=\sum_{q^{\prime}} T_{q q^{\prime}} M_{q^{\prime}}(t)+B_{q}
$$

então, a evoulução temporal de $\left\langle M_{q}(t) M_{q}\left(t^{\prime}\right)\right\rangle$ é determinada por

$$
\frac{d\left\langle M_{q}(t) M_{q}\left(t^{\prime}\right)\right\rangle}{d t}=\sum_{q^{\prime}} T_{q q^{\prime}}\left\langle M_{q}(t) M_{q}\left(t^{\prime}\right)\right\rangle+B_{q}\left\langle M_{q}\left(t^{\prime}\right)\right\rangle .
$$

Portanto, aplicando o teorema no primeiro e segundo termo da eq.(2.32), mediante as eqs. (2.20) e (2.21), obtemos

$$
\frac{d\langle\mathfrak{C}(t+\tau, t)\rangle}{d \tau} d \tau=\left\langle\frac{d x(t+\tau)}{d \tau} x^{\dagger}(t)\right\rangle d \tau+A_{T}\langle x(t+\tau)\rangle\left\langle x^{\dagger}(t)\right\rangle d \tau-y_{0}\left\langle x^{\dagger}(t)\right\rangle d \tau,
$$

obtendo finalmente

$$
\frac{d\langle\mathfrak{C}(t+\tau, t)\rangle}{d \tau}=-A_{T}\langle\mathfrak{C}(t+\tau, t)\rangle
$$

Dado que a transformada de Laplace é definida como

$$
\mathcal{L}[f(t)](s)=\int_{0}^{\infty} e^{-s t} f(t) d t
$$

então podemos transformar a eq.(2.36) em

$$
\mathcal{L}[\langle\mathfrak{C}(t+\tau, t)\rangle](s)=\left[s+A_{T}\right]^{-1}\langle\mathfrak{C}(t)\rangle,
$$

de modo que com a solução estacionária de $\mathfrak{C}(t)$ da eq. (2.31), podemos determinar a transformada de Laplace da matriz de covariânca.

\subsection{Espectro de ruído dos campos de prova e de controle}

A princípio, para calcular o espectro de ruído do campo de prova e de controle, deveríamos calcular transformada inversa de Laplace da eq. (2.38), obtendo assim a matriz de covariância, com a qual determinamos os espectros $S_{q q^{\prime}}(\omega)$. No entanto, usando a seguinte propriedade 


$$
\begin{aligned}
\int_{-\infty}^{\infty} e^{i \omega \tau}\langle\mathfrak{C}(t+\tau, t)\rangle d \tau & =\int_{-\infty}^{0} e^{i \omega \tau}\langle\mathfrak{C}(t+\tau, t)\rangle d \tau+\int_{0}^{\infty} e^{i \omega \tau}\langle\mathfrak{C}(t+\tau, t)\rangle d \tau \\
& =\int_{0}^{\infty} e^{-i \omega \tau}\left\langle\mathfrak{C}^{\dagger}(t+\tau, t)\right\rangle d \tau+\int_{0}^{\infty} e^{i \omega \tau}\langle\mathfrak{C}(t+\tau, t)\rangle d \tau \\
& =\mathcal{L}(s=-i \omega)+\mathcal{L}(s=i \omega)=2 \operatorname{Re}\{\mathcal{L}(s=-i \omega)\}
\end{aligned}
$$

na eq.(2.14), podemos determinar facilmente os espectros $S_{11}(\omega), S_{22}(\omega), S_{12}(\omega)$ e $S_{21}(\omega)$ de modo que:

$$
\begin{array}{r}
S_{11}(\omega)=2 \kappa^{2} \mathcal{E}_{1}^{2} \operatorname{Re}\left\{\mathcal{L}_{3,3}(-i \omega)+\mathcal{L}_{4,4}(-i \omega)-\mathcal{L}_{3,4}(-i \omega)-\mathcal{L}_{4,3}(-i \omega)\right\}, \\
S_{22}(\omega)=2 \kappa^{2} \mathcal{E}_{2}^{2} \operatorname{Re}\left\{\mathcal{L}_{5,5}(-i \omega)+\mathcal{L}_{6,6}(-i \omega)-\mathcal{L}_{5,6}(-i \omega)-\mathcal{L}_{6,5}(-i \omega)\right\} \\
S_{12}(\omega)=2 \kappa^{2} \mathcal{E}_{1} \mathcal{E}_{2} \operatorname{Re}\left\{\mathcal{L}_{3,5}(-i \omega)+\mathcal{L}_{4,6}(-i \omega)-\mathcal{L}_{3,6}(-i \omega)-\mathcal{L}_{4,5}(-i \omega)\right\} \\
S_{12}(\omega)=2 \kappa^{2} \mathcal{E}_{1} \mathcal{E}_{2} \operatorname{Re}\left\{\mathcal{L}_{5,3}(-i \omega)+\mathcal{L}_{6,4}(-i \omega)-\mathcal{L}_{6,3}(-i \omega)-\mathcal{L}_{5,4}(-i \omega)\right\}
\end{array}
$$

onde $\mathcal{L}_{i, j}(-i \omega)$ é a transformada de Laplace dos elementos $\{i, j\}$ da matriz de covariânca $^{4}$. Os espectros $S_{11}(\omega)$ e $S_{22}(\omega)$ correpondem ao espectro de cada um dos campos $\mathbf{E}_{1}(t)$ e $\mathbf{E}_{2}(t)$, respectivamente. O espectro de termos cruzados $S_{12}(\omega)$ permite calcular tanto a correlação do sistema, quanto o espectro de soma e diferença. A correlação normalizada entre os campos, numa frequência de análise específica, é definida como

$$
\mathcal{C}(\omega)=\frac{S_{12}(\omega)+S_{21}(\omega)}{2 \sqrt{S_{11}(\omega) S_{22}(\omega)}},
$$

sendo nosso principal parâmetro a ser estudado, tanto teoricamente quanto experimentalmente. Por outro lado temos o espectro da soma $S_{s}(\omega)$ e da diferença dos campos $S_{d}(\omega)$ :

$$
\begin{aligned}
& S_{s}(\omega)=S_{11}(\omega)+S_{22}(\omega)+S_{12}(\omega)+S_{21}(\omega), \\
& S_{d}(\omega)=S_{11}(\omega)+S_{22}(\omega)-S_{12}(\omega)-S_{21}(\omega) .
\end{aligned}
$$

Com estes resultados descrevemos teoricamente o espectro de ruído de cada um dos campos após interagir com o meio atômico. Nosso interese é estudar a correlação (2.44) dos campos em condição de EIT e mostrar como este modelo de difusão de fase reproduz os resultados experimentais mostrados no capítulo 4.

\footnotetext{
${ }^{4}$ Os elementros da matriz de covariânça podem-se visualizar a partir da matriz na eq. (2.26)
} 


\section{CAPÍTULO 3}

\section{Preparação e controle experimental dos átomos frios}

Neste capítulo mostraremos as etapas mais importantes da preperação do experimento para fazer as medidas de ruído em configuração $\Lambda$ EIT. Na primera parte, faremos um resumo de como é feito o resfriamento e aprisionamento de átomos. Posteriormente mostraremos as características da nossa nuvem de átomos frios de Rubídio. Dado que estas medidas de EIT são suscetíveis à presença de campos magnéticos, mostraremos as características do nosso controle de desligamento e compensação dos campos magnéticos. Na última parte, descreveremos o esquema de medição de ruído e o processo de calibração do ruído Shot-noise para um estado coerente da luz, que será o ponto de referência para as medidas feitas no experimento descrito no capítulo 4.

\subsection{Estrutura hiperfina dos átomos de Rubídio}

O elemento químico usado no laboratório para fazer resfriamento atômico é o Rubídio, sendo este um dos elementos principais usados no desenvolvimento de técnicas de resfriamento e aprisionamento, e com o qual foram obtidos os primeros condensados de Bose-Einstein [9]. Este elemento é um átomo hidrogenóide, cujo número atômico é 37 e pertence à família dos alcalinos (1A), descoberto por Bunsen e Kirchkoff em 1861. Existem dois isótopos do Rubídio: o Rubídio $85\left({ }^{85} \mathrm{Rb}\right)$, que corresponde a $72.2 \%$ do Rubídio encontrado na natureza e Rubídio $87\left({ }^{87} \mathrm{Rb}\right)$, que corresponde a $27.8 \%$ restante. Os spins nucleares para os dois isótopos são $I_{85}=\frac{5}{2}$ e $I_{87}=\frac{3}{2}$.

Especificamente, usamos ${ }^{85} \mathrm{Rb}$ para fazer o resfriamento e aprisionamento de átomos, usando as transições da linha D2, cujo comprimento de onda é $\lambda=780,241 \mathrm{~nm}$ 


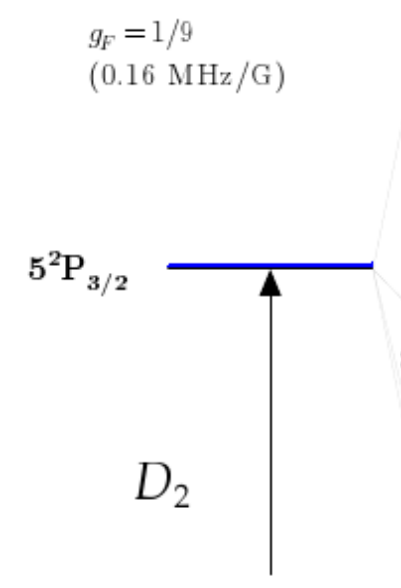

$780.241368271(27) \mathrm{nm}$ $384.230406373(14) \mathrm{THz}$
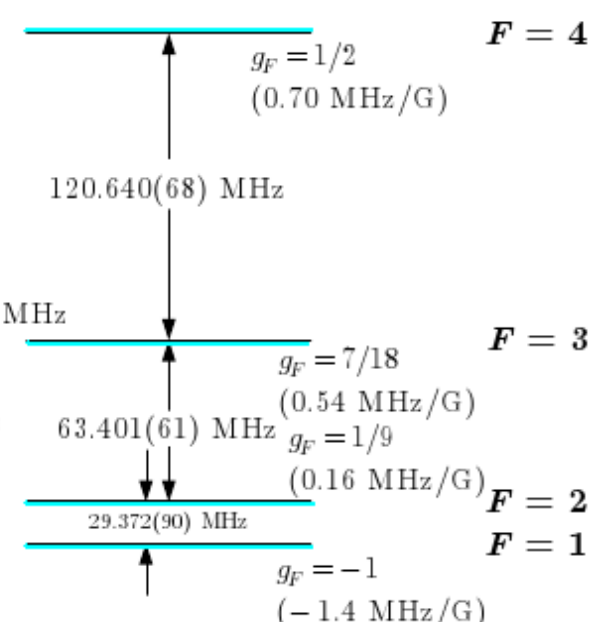

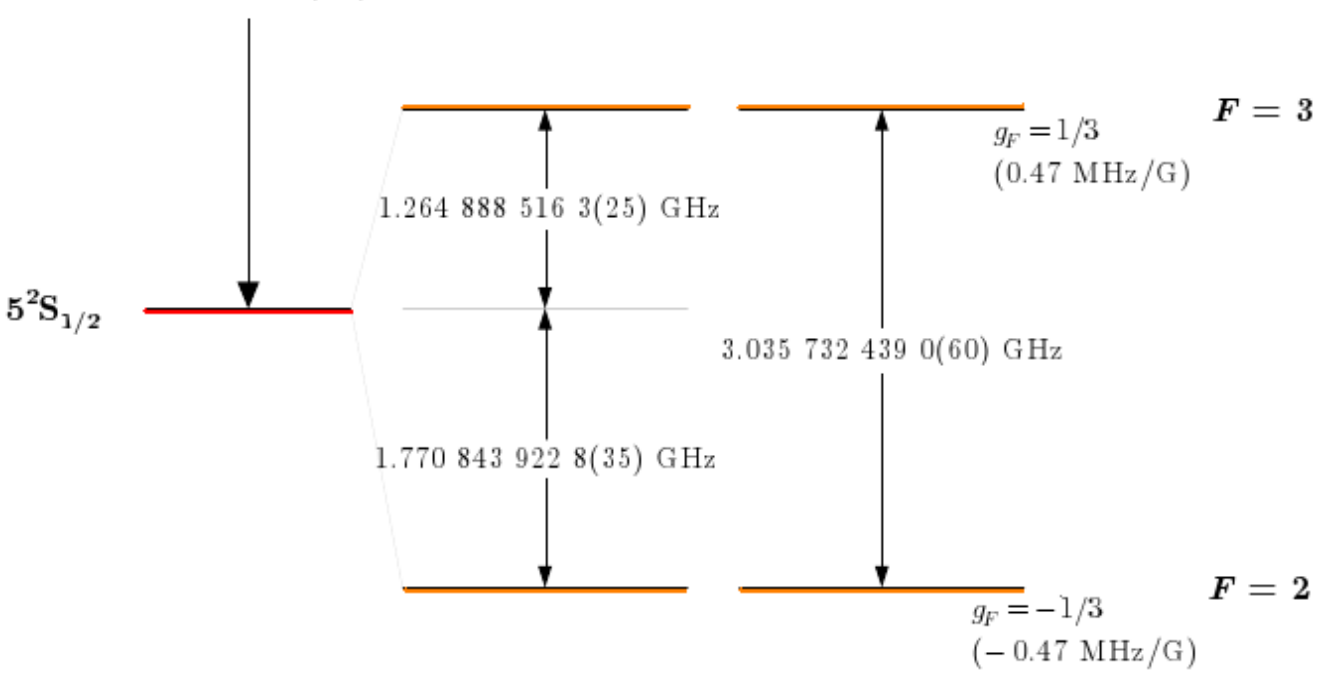

Figura 3.1: Estrutura hiperfina da linha D2 do Rubidio 85 [44].

(ver figura 3.1). O estado fundamental $\left({ }^{5} S_{1 / 2}\right)$ possui um momento angular $L=0$, spin $\frac{1}{2}$ e momento angular total $J=\frac{1}{2}$. Entretanto, o estado excitado $\left({ }^{5} P_{3 / 2}\right)$ possui momento angular $L=1$, spin $\frac{1}{2}$ e momento angular $J=\frac{3}{2}$. Cada um destes estados se desdobram em estados hiperfinos $F$, que correspondem a uma superposição do momento angular orbital, do spin eletrônico e do spin nuclear, tal que $F=L+S+I$. Os estados $F$ são estados degenerados, de modo que os sub-níveis Zeeman descritos por $m_{F}$ são definidos no intervalo $-F \leq m_{F} \leq F$. Estas transições atômicas são acessíveis por meio de lasers de Diodo, comumente usado nos laboratórios de Física Atômica. 


\subsection{Resfriamento e aprisionamento de átomos neu- tros}

O resfriamento de átomos mediante laser baseia-se no efeito da força radiativa que sofrem os átomos, pela absorção e emissão de luz. Isto é, os átomos com velocidade $v$ interagem com um feixe de luz em direção oposta, como mostra a figura 3.2.a, de
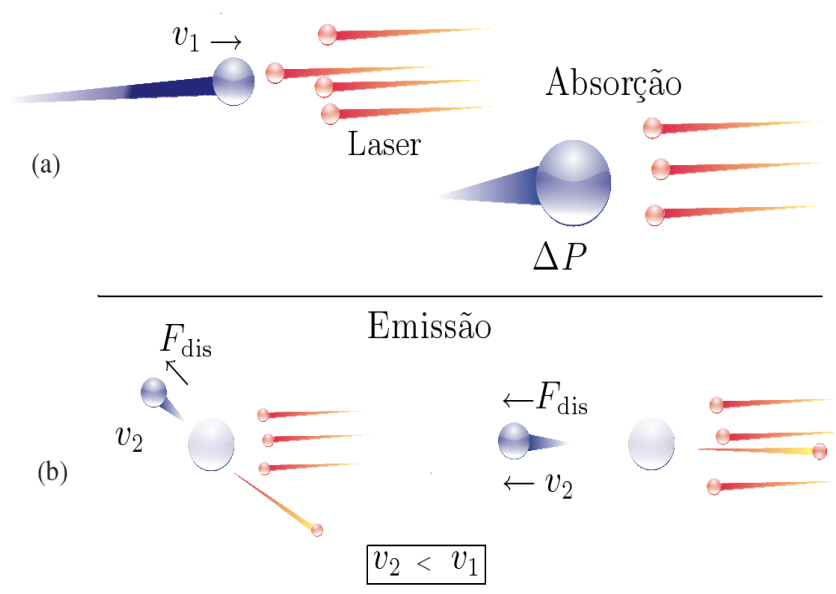

(c)
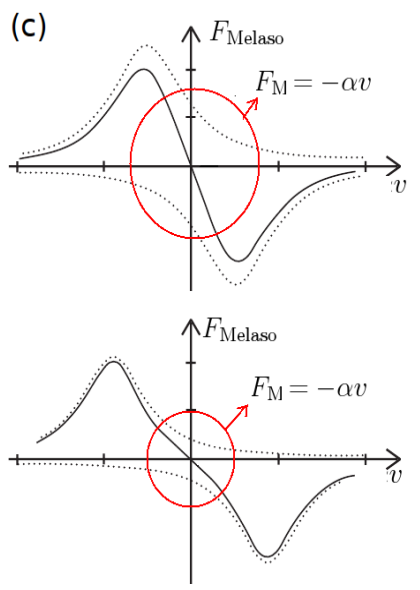

Figura 3.2: (a) Representação de um átomo com velocidade incial $v$, absorvendo um fóton, mudando assim seu momento. (b) Representamos o átomo emitindo um fóton em direção à luz incidente ou em direção aleatória, sofrendo uma força dispersiva. (c) Força de resfriamento em função da velocidade para uma dessintonia $\delta=-\Gamma / 2$ (acima) e $\delta=-\Gamma$ (abaixo). Para baixas velocidades, observa-se um comportamento linear da força de resfriamento.

modo que eles absorvem luz sofrendo trocas de momento em direção oposta $\Delta P$, com os fótons incidentes. Posteriormente eles emitem fótons em direções arbitrárias, criando, em média, uma variação no momento dos átomos representada como uma força de dispersão (figura 3.2.b).

Dado que os átomos possuem uma velocidade $v$, o efeito Doppler ocorre, induzindo um deslocamento na frequência vista do referencial do átomo, diminuindo assim a seção de choque e, portanto, o efeito da força de dispersão $F_{\text {dis }}$ diminui conjuntamente $[40,45,46]$. Esta força está dada por

$$
F_{\text {dis }}(\delta-k v)=\hbar k \frac{\Gamma}{2} \frac{I / I_{\text {Sat }}}{1+I / I_{\text {Sat }}+4(\delta-k v)^{2} / \Gamma^{2}}
$$

Pode-se compensar a perda da probabilidade de absorção, usando dois feixe de luz contrapropagantes, tal que a força efetiva que atua no átomo é uma força de amortecimento

$$
F_{\text {Melaço }}=F_{\text {dis }}(\delta-k v)-F_{\text {dis }}(\delta+k v) \approx-\alpha v, \quad k v \ll \Gamma
$$




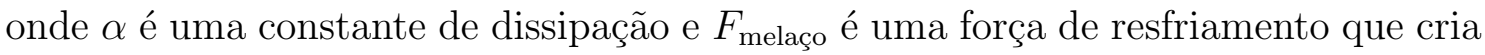
o Melaço ótico. A constante $\alpha$ depende da dessintonia na frequência do feixe de luz em relação com a transição atômica, tal que

$$
\alpha=4 \hbar k^{2} \frac{I}{I_{\mathrm{sat}}} \frac{-2 \delta / \Gamma}{\left[1+(2 \delta / \Gamma)^{2}\right]^{2}}, \quad \frac{I}{I_{\mathrm{sat}}} \ll 1
$$

sendo $I_{\text {Sat }}$ a intensidade para a qual a absorção atômica se satura. Dependendo do sinal da dessintonia $\delta$, a constante $\alpha$ pode ser positiva o negativa, tal que a força $F_{\text {dis }}$ vai frear o empurrar os átomos. Portanto, é necessário $\delta<0$ para resfriar os átomos $^{1}$ (dessintonia para o vermalho). A figura 3.2.c [45] mostra a força $F_{\text {Melaço, }}$ que cria o melaço ótico em função da velocidade, para uma dessintonia $\delta=-\Gamma / 2$. O

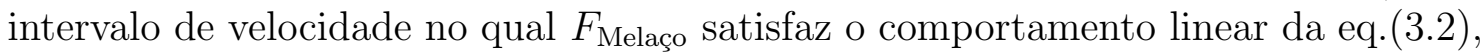
é maior que no caso em que a dessintonia é $\delta=-\Gamma$. Neste intervalo de velocidades, a força comporta-se como uma força de amortecimento.

No laboratório fizemos o resfriamento mediante três feixes contrapropagantes nas direções $x, y$ e $z$, como mostramos na figura 3.3. O cruzamento dos três feixes determina uma região no espaço onde existe uma alta probabilidade dos átomos serem resfriados. No entanto, ainda não observamos uma nuvem de átomos frios. Embora o resfriamento seja feito, a densidade espacial não muda [40], pois é necessário um mecanismo de aprisionamento para que isso ocorra.
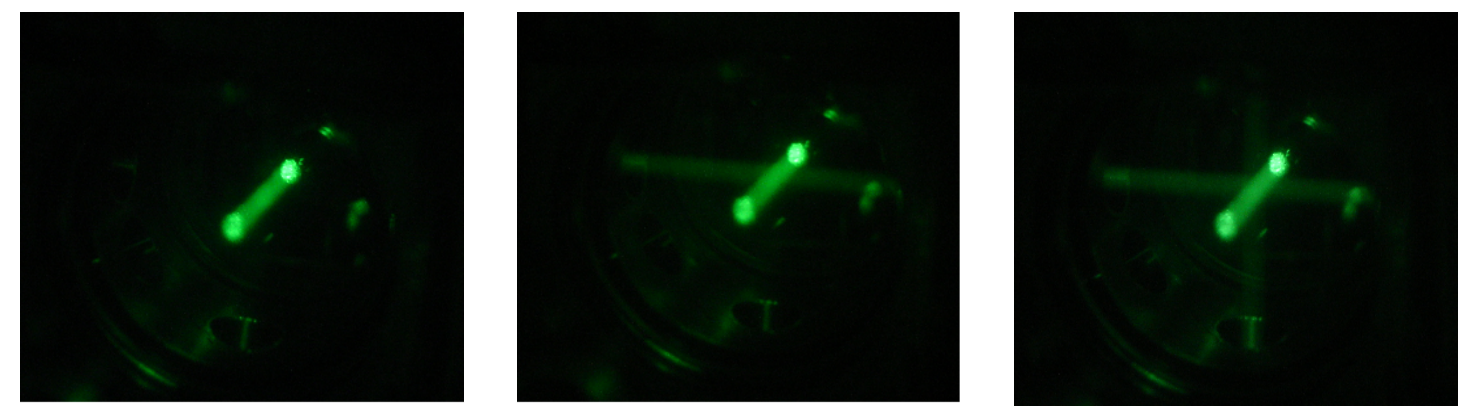

Figura 3.3: (Esquerda) Feixes contrapropagantes no eixo $\hat{y}$ ligados. (Centro) Feixes contrapropagantes no eixo $\hat{x}$ ligados. (Direita) Feixes contrapropagantes no eixo $\hat{z}$ ligado.

Numa Armadilha Magneto-Ótica (MOT, sigla em inglês) é possível fazer o resfriamento e o aprisionamento de átomos. O aprisionamento dos átomos é feito criando-se uma inomogeneidade espacial, mediante um campo magnético, induzindo uma aborção preferencial no espaço dos feixes contrapropangantes encarregados do resfriamento. Esta absorção preferencial no espaço faz com que os átomos sejam aprisionados.

\footnotetext{
${ }^{1}$ Este resfriamento tem um limite denominado limite Doppler de resfriamento devido à difusão térmica $[45,46,47]$
} 
A figura 3.4.a mostra o mecanismo de aprisionamento. Para isto, consideramos que os feixes de resfriamento possuem polarizações circulares opostas $\sigma_{+}$e $\sigma_{-}$, os quais vão acoplar transições entre níveis Zeeman. Por exemplo, consideramos o estado fundamental $F=0$ e o estado excitado $F=1$, de modo que o feixe com polarização $\sigma_{+}$acopla a transição do estado $\left|F_{f}, m_{f}=0\right\rangle$ ao estado $\left|F_{e}, m_{e}=1\right\rangle$ e o feixe com polarização $\sigma_{-}$acopla a transição do estado $\left|F_{f}, m_{f}=0\right\rangle$ ao estado $\left|F_{e}, m_{e}=-1\right\rangle$. Quando o campo magnético é aplicado, por exemplo $\mathbf{B}(z)=\frac{\partial B}{\partial z} z \hat{e}$,

(a)

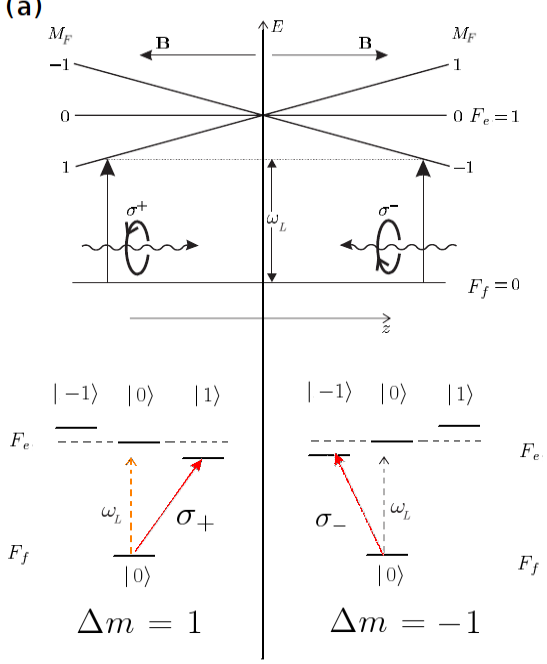

(b)

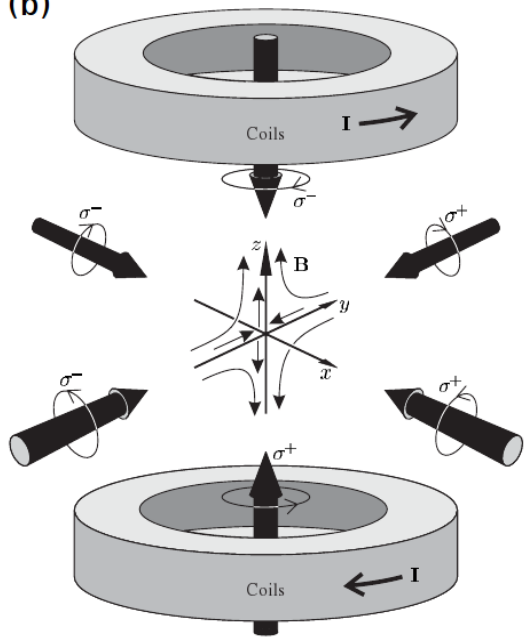

Figura 3.4: Imagem obtida de [45] (a) Mecanismo de aprisionamento. (b) Esquema experimental dos feixes de resfriamento e das bobinas que criam o campo magnético para aprisionar os átomos.

este quebra a degenerescência dos níveis Zeeman, induzindo uma dessintonia na frequência do laser visto pelo átomo, de modo que o átomo absorve o feixe com polarização $\sigma_{+}$, então $\Delta m=1$, sofrendo uma força $F_{\text {dis }}^{\sigma_{+}}$em direção ao zero do campo $B$, mas se ele absorve o feixe $\sigma_{-}$, então $\Delta m=-1$ sofrendo uma força $F_{\text {dis }}^{\sigma_{-}}$de novo em direção ao zero do campo $B$. Mediante este mecanismo, a força resultante atuante sobre os átomos na direção $z$ é dada por

$$
F_{\mathrm{MOT}}=F_{\text {dis }}^{\sigma_{+}}(\delta-k v-\beta z)-F_{\text {dis }}^{\sigma_{-}}(\delta+k v+\beta z)
$$

tal que no caso onde $\beta z \ll \Gamma$ e $k v \ll \Gamma$, temos

$$
F_{\mathrm{MOT}} \approx-\alpha v-\frac{\alpha \beta}{k} z
$$

onde

$$
\beta=\frac{g \mu_{B}}{\hbar} \frac{d B}{d z}, \quad g=g_{F}(e) m_{F(e)}-g_{F}(f) m_{F(f)}
$$


sendo $g$ um fator giromagnético efetivo e $\frac{d B}{d z}$ o gradiente do campo magnético usado para aprisionar. Mediante a eq.(3.5), mostramos que é possível resfriar e aprisionar átomos, como uma partícula em presença de força de amortecimento e força restauradora, respectivamente (como acontece no Oscilador amortecido). A figura 3.4.b mostra o esquema experimental de uma MOT.

O campo magnético que temos no laboratório para gerar o aprisionamento, é produzido por duas bobinas em cofiguração Anti-Helmoltz, com 550 espiras cada e uma corrente de $I=1.5 \mathrm{~A}$ (pode chegar até $I=2.5 \mathrm{~A}$ ), tal que o gradiente de campo está entre 15 e 20 Gauss/cm. Na figura 3.5 mostramos a nuvem de átomos frios formada após do campo magnético ser ligado.

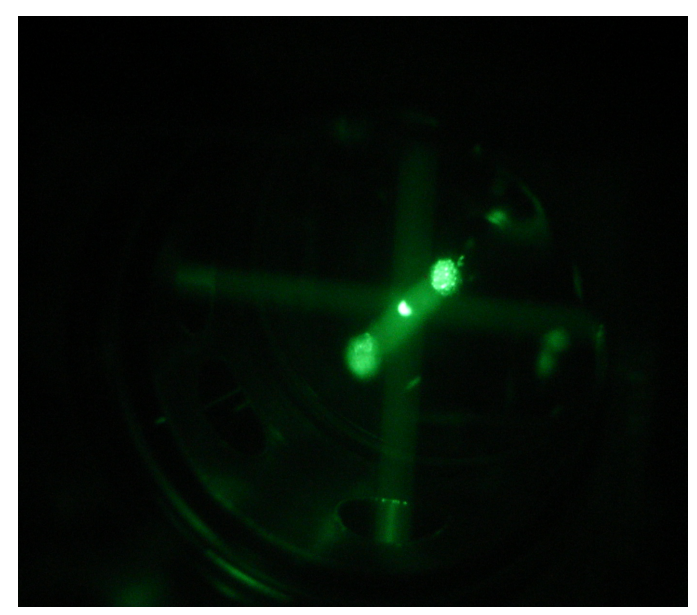

Figura 3.5: Nuvem de átomos frios de Rubídio 85 no LMCAL

Para fazer o resfriamento, usamos laser de Diodo num comprimento de onda de $780.2 \mathrm{~nm}$, que acopla a linha D2 do Rubídio 85. A frequência do laser de resfriamento é travada na transição $F=3\left({ }^{5} S_{1 / 2}\right) \rightarrow F=4\left({ }^{5} P_{3 / 2}\right)$. No entanto, é necessário o controle da população no estado $F=3$, porque existe a possibilidade da população ser bombeada para o estado $F=2$, assim usamos mais um laser com frequência travada na transição $F=2\left({ }^{5} S_{1 / 2}\right) \rightarrow F=3\left({ }^{5} P_{3 / 2}\right)$, para rebombear a população no estado $F=3$.

\subsection{Temperatura e número de átomos aprisona- dos}

A temperatura da nuvem é medida a partir da técnica de imagens de expansão livre dos átomos, como mostra a sequência de imagens da figura 3.6. Mediante estas 
imagens de fluorescência da nuvem em expansão livre, calcula-se a velocidade de expansão, fazendo um ajuste Gaussiano para o perfil de intensidade projetado para $x$, como mostra a figura 3.7.a. Inicalmente o diâmetro da nuvem é $D=4.0 \pm 0.7 \mathrm{~mm}$. Para vários tempos de expansão , a posição relativa dos átomos ao centro de massa,
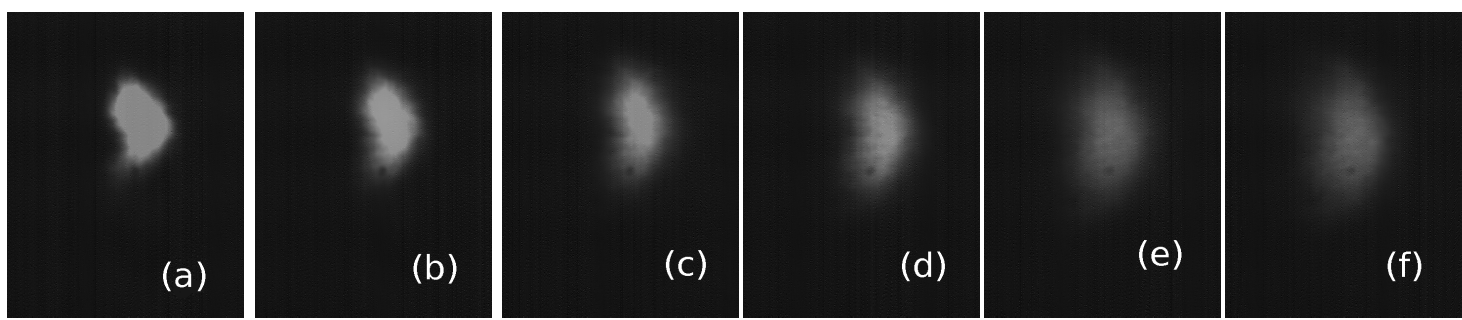

Figura 3.6: Imagens de expansão livre da nuvem atômica, para tempos de expansão (a) $\tau=2 \mathrm{~ms}$, (b) $\tau=4 \mathrm{~ms}$, (c) $\tau=6 \mathrm{~ms}$, (d) $\tau=8 \mathrm{~ms},(\mathbf{e}) \tau=10 \mathrm{~ms}$ e (f) $\tau=12 \mathrm{~ms}$.

é dada por

$$
\left\langle x_{\mathrm{rel}}^{2}\right\rangle=\left\langle v_{x}^{2}\right\rangle t^{2}
$$

de modo que determinamos o desvio padrão do ajuste Gaussiano ${ }^{2}$ de cada tempo, obtendo um gráfico de $\sigma_{x}^{2}=\left\langle x_{\text {rel }}^{2}\right\rangle$ em função do tempo de expansão, como mostra a figura 3.7.b, do qual obtemos a velocidade $\left\langle v^{2}\right\rangle$.
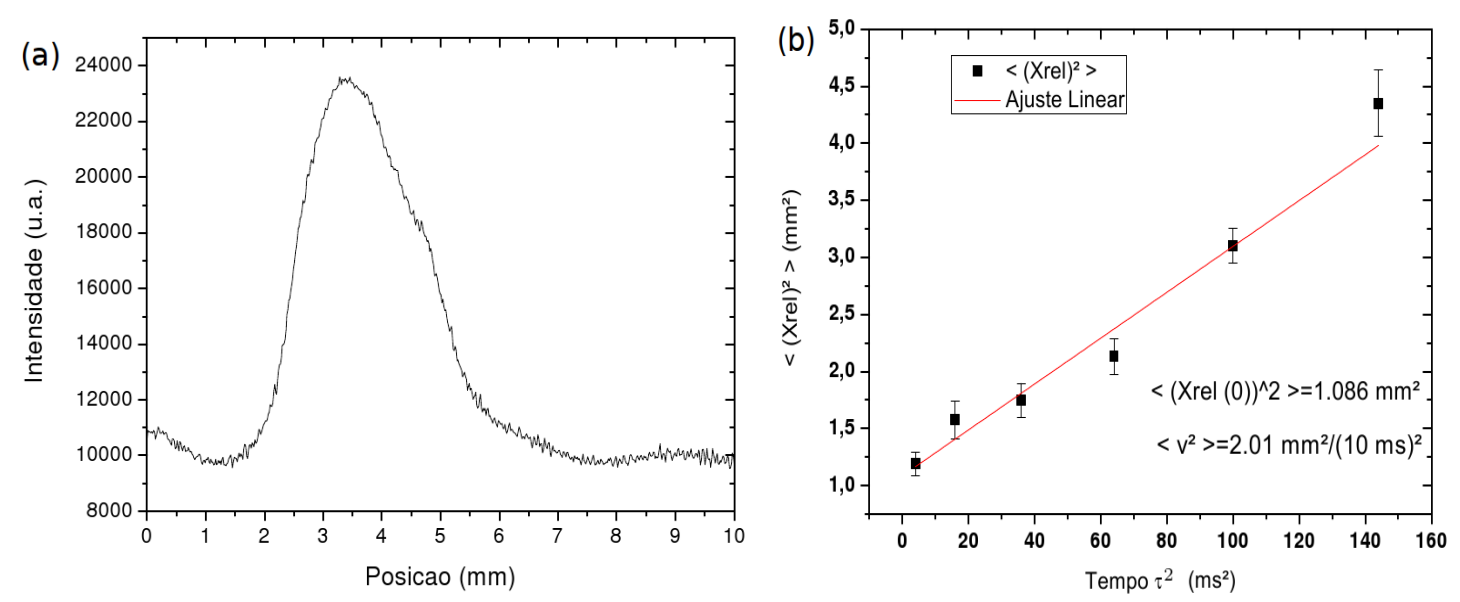

Figura 3.7: (a)Perfil de intensidade da nuvem atômica, projetado sobre um dos eixos da imagem. (b) Posição dos átomos $\left\langle X_{\text {rel }}^{2}\right\rangle$ relativo ao centro da nuvem en função do tempo $\tau^{2}$.

A temperatura é calculada a partir do Teorema da equipartição $T_{x}=\left(m / k_{B}\right)\left\langle v_{x}^{2}\right\rangle$, sendo $\left(m / k_{B}\right) \approx 1.02 \times 10^{-2} K \cdot s^{2} / m^{2}$, tal que

$$
T=205(15) \mu \mathrm{K}
$$

${ }^{2}$ Este desvio padrão é resultado de uma média de 10 medidas por tempo de expansão. 
Esta estimativa mostra que a temperatura da nuvem está acima do limite Doppler $T_{D}=143 \mu \mathrm{K}$. Anteriormente se chegou a ter aproximadamente $150 \mu \mathrm{K}$ no laboratório, no entanto, isso muda em cada experiência dependendo da dessintonia e do alinhamento dos feixes de resfriamento.

Além da medida de temperatura, nas imagens de expansão da figura 3.6, podemos observar que, depois que os feixes de resfriamento são desligados, a nuvem possui uma densidade considerável até o tempo de vôo de $6 \mathrm{~ms}$. Este tempo é o limite máximo dentro do qual as medidas de EIT são feitas.

Para estimarmos o número médio de átomos na nuvem, coletamos uma fração da fluorescência emitida pela nuvem, utilizamos uma lente convergente e focalizamos a nuvem sobre um fotodetector, como mostra a fgura 3.8. O número de átomos

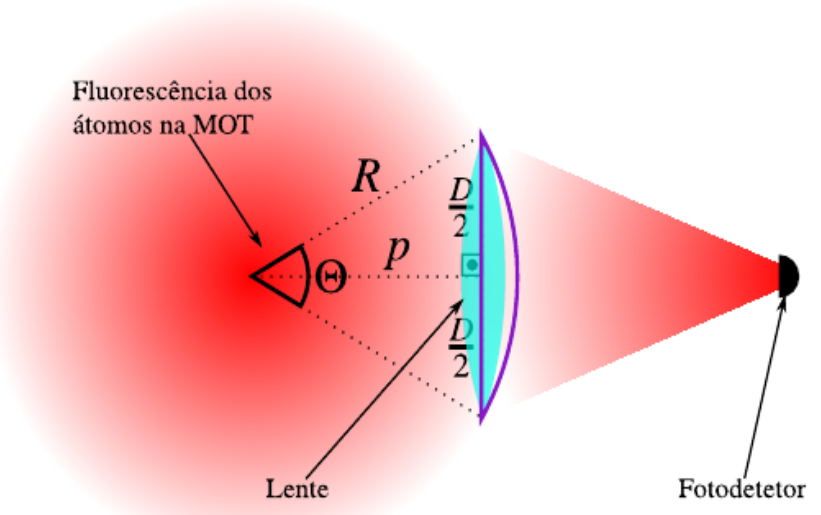

Figura 3.8: Esquema experimental para medir o número de átomos, mediante uma lente focando parte da luz emitida pelos átomos num fotodetector.

é calculado como em [48], mediante a relação de potência capturada pela lente de tamanho $D$, a distância da lente à nuvem $p$ e a intensidade $I$ dos feixes com os quais fazemos o resfriamento, tal que

$$
N_{\text {atom }}=\frac{4}{\Gamma} \frac{\lambda}{h c}\left(\frac{V S_{P D}}{\frac{1}{2}-\frac{p}{\sqrt{(2 p)^{2}+D^{2}}}}\right)\left(\frac{\left(\frac{2 \delta}{\Gamma}\right)^{2}+\frac{I}{I_{S a t}}+1}{\frac{I}{I_{S a t}}}\right)
$$

onde $V \sim 500 \mathrm{~m} V$ é a tensão medida no fotodetector, $S_{P D}=6 \times 10^{-7} \mathrm{~W} / \mathrm{V}$ corresponde à sensiblidade de potência e tensão $(P / V)$ calibrada para nosso fotodetector. A posição da lente em relação a nuvem é $p=19 \mathrm{~cm}$, e o diâmetro é $D=3 \mathrm{~cm}$. A intensidade dos feixes com os quais fizemos o refriamento dos átomos é $I=30.4+9.76+9.55 \mathrm{~mW} / \mathrm{cm}^{2}$ e $I_{\text {Sat }}=1.6 \mathrm{~mW} / \mathrm{cm}^{2}$, considerando que possuem uma dessintonia $\delta=10 \mathrm{MHz}$. Usando os valores conhecidos para $\lambda=780.24 \mathrm{~nm}$, 
$c=3 \times 10^{8} \mathrm{~m} / \mathrm{s}$ e a largura natural $\Gamma=5.8 \mathrm{MHz}$ dos átomos de Rubídio, temos ao final

$$
N_{\text {atom }} \approx 7 \times 10^{7} \text { átomos }
$$

\subsection{Desligamento do campo magnético de apriso- namento}

Um parâmetro que deve ser muito bem controlado nas medidas de EIT é o campo magnético residual, cuja origem provêem de diferentes fontes internas e externas do sistema de resfriamento e aprisionamento dos átomos. Dado que no esquema para obter EIT usamos dois feixes com polarizações circulares opostas, acoplando níveis Zeeman, que são susceptíveis a campos magnéticos, obtemos transparência do meio na condição Raman $\delta_{R}=0$. A presença de algum campo magnético residual (por exemplo: a bomba iônica próxima da nuvem, o campo magnético da terra, etc.), quebra a degenerescência dos estados Zeeman, de modo que a condição Raman já não é satisfeita, pois o laser fica em dessintonia com os níveis atômicos, cancelando o efeito de transparência.

Considerando a expansão do campo magnético ao redor da posição dos átomos, temos que

$$
B(z)=B(0)+\left.\frac{d B}{d z}\right|_{z=0} \Delta z_{\text {atom }}+\mathcal{O}\left(\Delta z_{\text {atom }}^{2}\right)
$$

onde o primeiro termo esta fortemente relacionado com os campos espúreos, e o segundo termo é o gradiente de campo magnético que provêem das bobinas antiHelmoltz, usadas para o aprisionamento de átomos. O objetivo é cancelar o campo magnético total numa região do espaço do tamanho da nuvem $\Delta z_{\text {atom }}$, durante o tempo de medida de EIT para evitar sair da condição Raman, como mostra a figura 3.9. Para isso, devemos compensar os campos espúreos $B_{\text {espúreo }}(0)$ mediante uma bobina em configuração Helmoltz produzindo um campo $B_{\text {comp }}$ constante no eixo $z$, de modo que $B(0)=B_{\text {espúreo }}(0)-B_{\text {comp }}(0)=0$. Por outro lado, precisamos desligar rapidamente as bobinas de aprisionamento durante a medida de EIT.

Dado que o tempo no qual é feita a medida de EIT é de aproximadamente 2ms$5 \mathrm{~ms}$, precisamos de um desligamento rápido do campo gerado pelas bobinas antiHelmoltz, de modo que reduza o efeito de quebra de degenerescência. No entanto, precisamos saber quão rápido estamos desligando o campo no ponto do espaço onde é formada a nuvem. Tecnicamente é impossível colocar um magnetômetro dentro da câmara de vácuo e medir o campo magnético. Para resolver esse problema técnico, usamos uma bobina de prova e a colocamos fora da câmara, sobre o eixo 


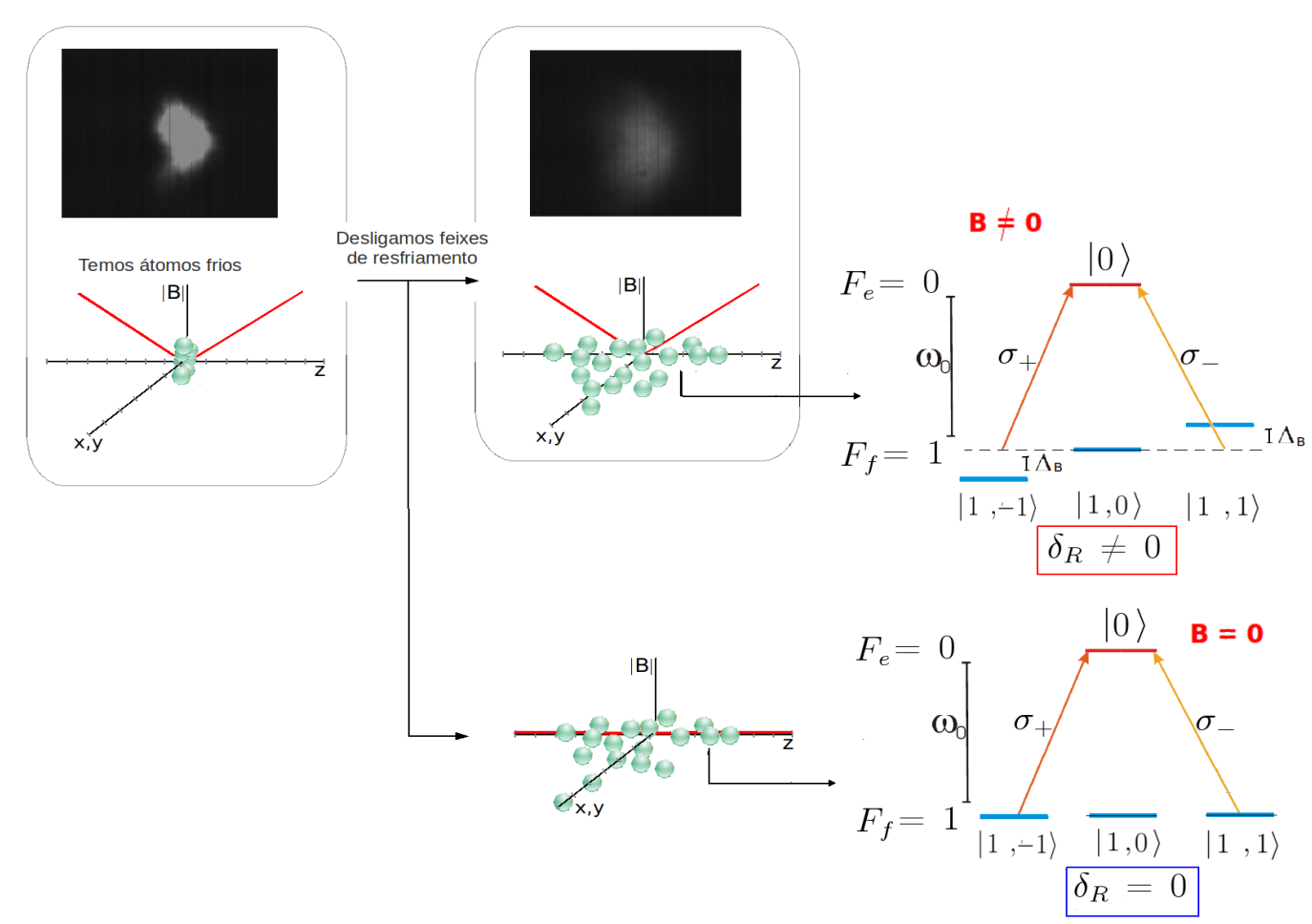

Figura 3.9: Processo de desligamento das bobinas de aprisionamento. Depois de desligarmos os feixes de resfriamento, os átomos se espalham, saindo da região onde o campo magnético é nulo, quebrando assim a degenerescência dos níveis fundamentais (parte superior). Mas desligando o campo magnético evitamos quebrar a degenerescência (parte inferior), estabelecendo assim ótimas condições para fazer as medições de ruído em configuração de EIT

das bobinas, para medir a tensão induzida nela, devido ao desligamento das bobinas como se mostra no gráfico da figura 3.10.a.

O sinal de tensão induzido na bobina de prova apresenta um platô de tensão durante 480ms após desligar as bobinas. Isto acontece, porque desligamos o campo magnético, descarregando a corrente das bobinas num varistor, de modo que a tensão nele permanece constante. Portanto, a tensão na bobina de prova mantem-se constante devido à variação linear do fluxo magnético, durante este tempo de descarga no varistor. Depois desta descarga rápida, a corrente nas bobinas descarga de forma exponencial sobre um resistor.

A partir deste sinal de tensão, usando a Lei de Faraday-Lenz e integrando a equação de indução, obtemos o campo magnético

$$
B=-\int_{0}^{t_{1}} V(t) d t
$$

onde consideramos que a área da bobina de prova é suficientemente pequena para 
não medir variações do campo, numa região do espaço maior do que o tamanho da nuvem. Isto faz com que o fluxo do campo magnético $\Phi$ seja proporcional ao campo magnético $B$.
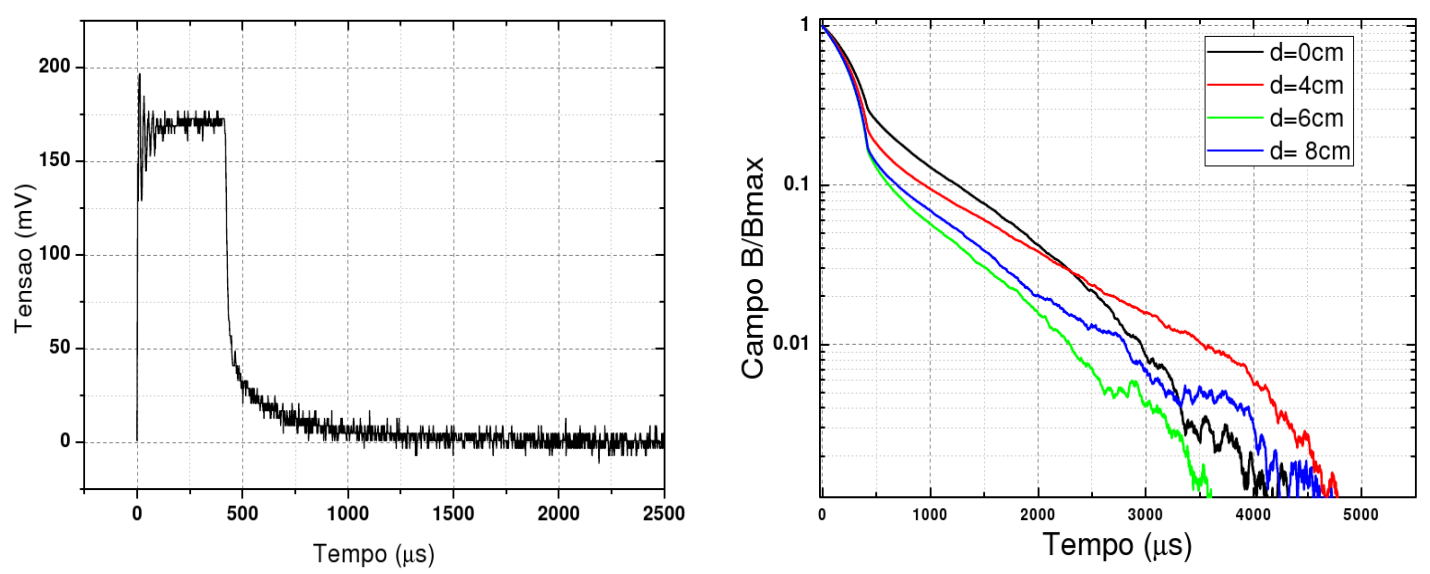

Figura 3.10: (a) Tensão induzida na bobina de prova, para uma corrente $I=0.8$ na bobina anti-Helmoltz. (b) Campo magnético normalizado pelo valor máximo do campo, para várias posições da bobina de prova.

Para um valor de corrente de $I=0.8 \mathrm{~A}$ nas bobinas anti-Helmoltz obtemos, a partir da eq. (3.12), o campo magnético para várias posições sobre o eixo, como mostra a figura 3.10.b. Construindo um gráfico semelhante à figura 3.10.b para as correntes $I=0.8 \mathrm{~A}, I=1.5 \mathrm{~A}$ e $I=2 \mathrm{~A}$, fizemos o gráfico do campo magnético (normalizado pelo valor máximo $B_{\max }$ ) em função da posição $z$, como e mostra a figura 3.11. Nesse gráfico observamos que, tanto o campo magnético, quanto o $\frac{d B}{d z}$, não podem ser medidos diretamente na região dos átomos $z \in[-5 \mathrm{~cm}, 5 \mathrm{~cm}]$ limitada pela câmara. No entanto, fazendo um ajuste dispersivo, logramos determinar uma expressão para o campo, a partir do qual obtemos o gradiente na posição $z=0$. Calculando o gradiente para os tempos medidos, obter uma noção aproximada de quão rápido desligamos o campo magnético. Na figura 3.11 vemos como o gradiente decai mais rápido no tempo para uma corrente $I=0.8 \mathrm{~A}$ do que para uma corrente de $I=2 \mathrm{~A}$. Porém, usar uma corrente de $I=0.8 \mathrm{~A}$, implica ter um menor número de átomos. Por esta razão usamos $I=1.5 \mathrm{~A}$, com a qual obtemos um número considerável de átomos, na eq.3.10, e uma diminuição de até $90 \%$ do gradiente do campo em 1ms, aproximadamente.

Esta técnica para medir o campo magnético é usada como primeira abordagem para obtenção aproximada do tempo de desligamento. No entanto, com o mesmo meio atômico poderíamos medir diretamente o valor do campo magnético, levando em conta o efeito linear magneto-ótico [49], em que a largura do pico pode estar associada ao campo magnético, tal que $2 g \mu_{B} B=\hbar \Gamma_{\text {EIT }}$, de modo que, determinando a largura $\Gamma_{\text {EIT }}$, podemos obter o campo magnético na região do espaço onde se 

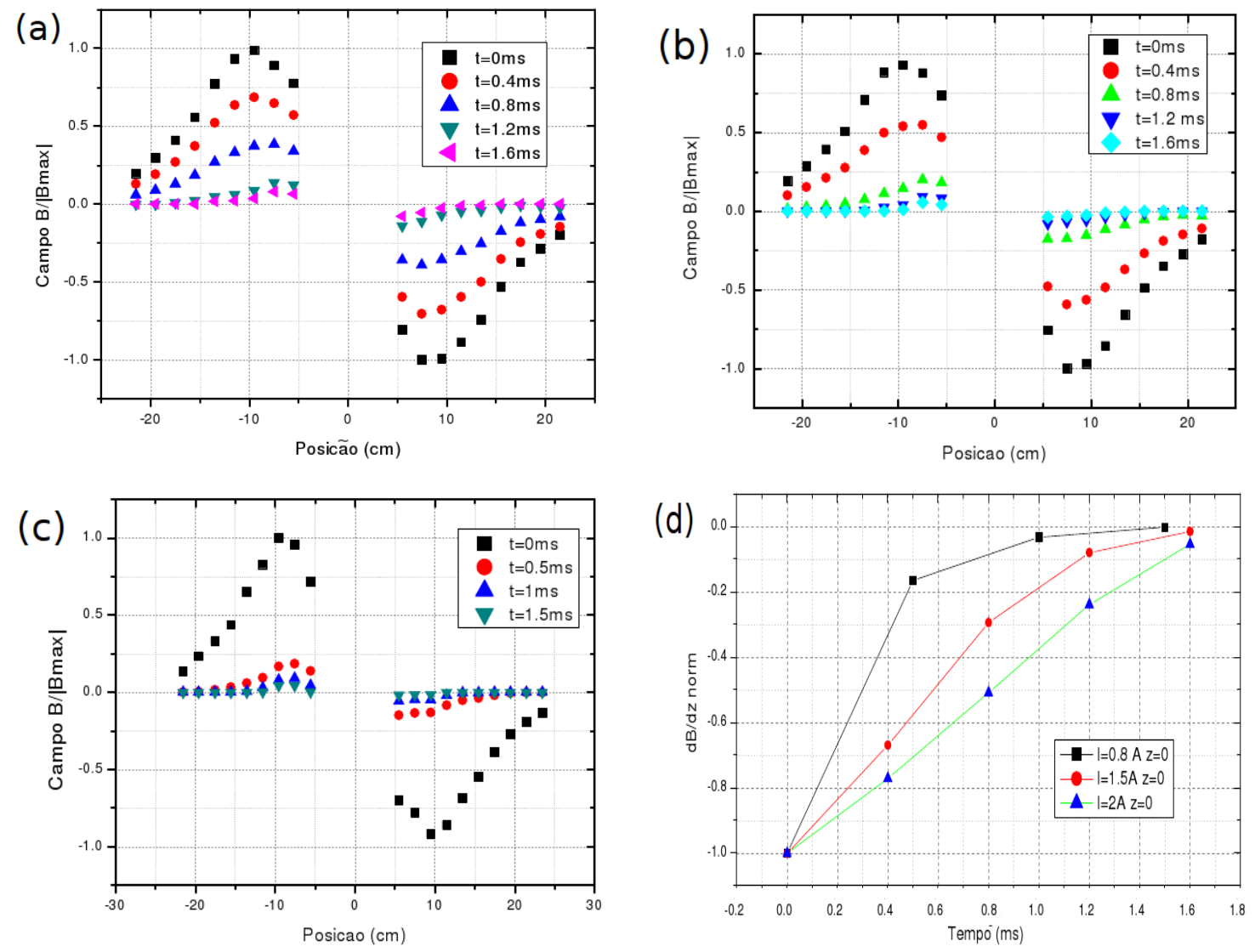

Figura 3.11: Campo magnético normalizado pelo valor máximo para: (a) Corrente $I=0.8 \mathrm{~A}$. (b) Corrente $I=1.5 \mathrm{~A}$. (c) Corrente $I=2 \mathrm{~A}$. (d) Extrapolação do gradiente do campo magnético no centro da câmara, normalizado pelo valor máximo, em função do tempo

forma a nuvem. No próximo capítulo, mostraremos a importância do desligamento do campo magnético sobre o espectro de EIT.

\subsection{Esquema de deteção e aquisição de dados}

Uma parte essencial do experimento é a deteção dos campos eletromagnéticos após da interação com o meio atômico. Uma vez que a deteção é feita, demodulamos as flutuações de intensidade (fotocorrente) numa frequência de análise dada, e empregamos um sistema de aquisição digital para armazenar os dados diretamente no computador. Este esquema é diferente do caso análogico, em que o ruído é medido mediante o Analisador de Espectros para várias frequências de análise.

Para medir a intensidade média e o ruído da luz, utilizamos fotodiodos, onde um fluxo regular de intensidade de luz gera uma corrente elétrica regular. A relação 
linear entre a intensidade do feixe incidente e o fluxo de corrente elétrica é dada por

$$
\bar{i}_{\text {elet }}=G(\omega) I_{\text {inc }}(\omega), \quad G(\omega)=\frac{e A}{\hbar \omega}
$$

onde $G(\omega)$ é o ganho do detector, e é a carrega elétrica e $A$ é a área do detetor ideal. Nas medidas feitas no laboratório, usamos fotodetectores FND 100 da EG\& G. que possuem uma eficiência quântica superior a $86 \%$.

O sinal do fotodetector corresponde ao sinal de entrada de um circuito que dá acesso à componente DC, ou seja, o sinal médio da intensidade detectada e ao sinal de alta frequência que corresponde à componente $\mathbf{H F}$, o qual é amplificado para logo passar para uma etapa de demodulção. O demodulador mistura o sinal de entrada com o sinal de um Oscilador Local (LO) com frequência $\Omega$, produzido por um gerador de funções Hewlett Packard (HP). O sinal demodulado passa por um amplificador com um filtro passa-baixa de $300 \mathrm{kHz}$, produzindo assim na sua saída, uma imagem do sinal eletrônico na frequência de análise $\Omega$ em baixa frequência, com uma largura de banda de $600 \mathrm{kHz}$.

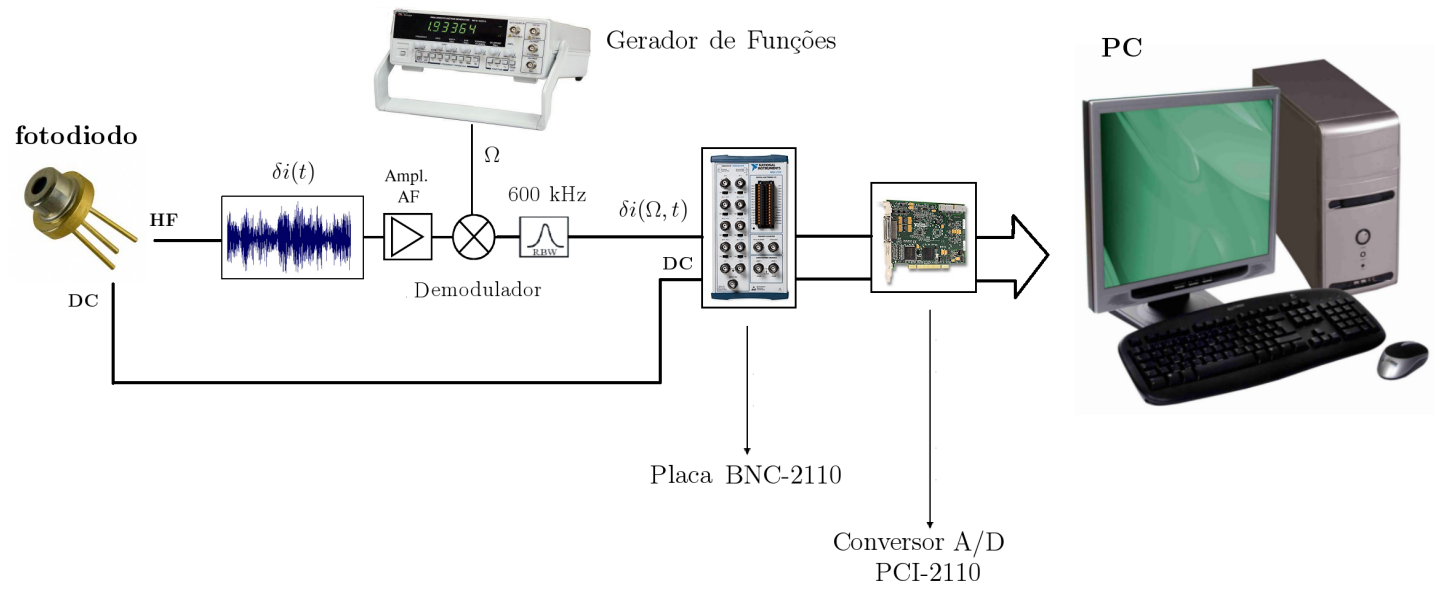

Figura 3.12: Processo de deteção. Usamos o fotodetector que fornece uma saida de sinal DC e Sinal HF. O sinal HF é demodulado numa taxa de $600 \mathrm{kHz}$. Os dois sinais saõ adquiridos pela placa BNC-2110 para logo serem convertidos em sinais digitais, mediante o conversor A/D. Finalmente todos os dados são armazenados diretamente no computador.

Este esquema de demodulação já foi usado no grupo [50], pois permite adquirir tanto o sinal DC como o sinal de alta frequência ao mesmo tempo, mediante uma placa de aquisição de dados da National Instruments (modelo BNC-2110), conectada a uma placa conversora A/D (análogo-digital) da mesma empresa (modelo PCI2110) que adquire os dados diretamente no computador (PC) mediante o programa LabView $^{3}$ (ver características do programa no apêndice A).

\footnotetext{
${ }^{3} \mathrm{O}$ ruído do campo pode ser medido tradicionalmente mediante um analisador de espectros, no entanto o esquema de demodulação permite um fácil acesso e tratamento de dados.
} 
A calibração do ruído dos detectores é feita a partir de deteção homodina balanceada. Em geral, esta pode fornecer as flutuações de fase e amplitude do campo eletromagnético, misturando dois feixes da mesma frequência usando um divisor de feixe, como se mostra na figura 3.13 (Esquerda). Consideremos os campos $\hat{a}$ e $\hat{b}$
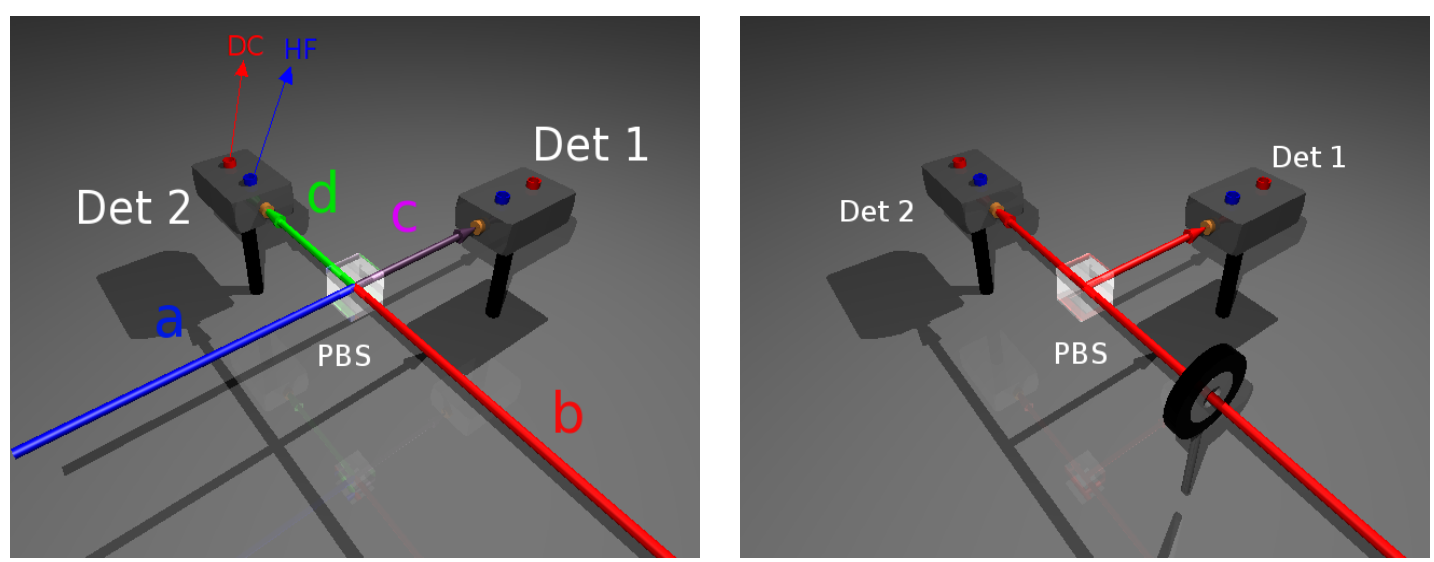

Figura 3.13: (Esquerda) Esquema de deteção homodina balanceada. Embora os canais de entrada e saida representam-se com cores diferentes, experimentalmente o sinal e o LO devem ter a mesma frequência. (Direita) Esquema de deteção balanceda, com o qual pode-se determinar o Shot-noise.

incidindo num divisor de feixe com transmissão $|t|^{2}$ e reflexão $|r|^{2}$ iguais (deteção balanceada). Na saída deste, obtemos dois campos $\hat{c}$ e $\hat{d}$, dados por

$$
\begin{aligned}
& \hat{c}=\frac{1}{\sqrt{2}}(\hat{a}+\hat{b}) \\
& \hat{d}=\frac{1}{\sqrt{2}}(\hat{a}-\hat{b})
\end{aligned}
$$

onde o termo negativo na segunda equação corresponde à defasagem que ganha o feixe ao ser refletido. As intensidades medidas nos fotodetetores, a partir da eq. (3.13), levando as amplificações nos circuitos acoplados aos fotodetetores, temos

$$
\begin{aligned}
& \hat{I}_{1}=g \hat{d}^{\dagger} \hat{d} \\
& \hat{I}_{2}=g \hat{c}^{\dagger} \hat{c}
\end{aligned}
$$

onde $g$ é uma constante de ganho dos detetores (leva em conta amplificações dos circuitos eletrônicos). Substituindo as eqs.(3.14) e (3.15) nestas equações, obtemos as intensidades de soma e diferença

$$
\begin{aligned}
& \hat{I}_{+}=\hat{I}_{1}+\hat{I}_{2}=g\left(\hat{a}^{\dagger} \hat{a}+\hat{b}^{\dagger} \hat{b}\right) \\
& \hat{I}_{-}=\hat{I}_{1}-\hat{I}_{2}=g\left(\hat{a}^{\dagger} \hat{b}+\hat{b}^{\dagger} \hat{a}\right) .
\end{aligned}
$$


Para determinar o ruído dos campos, precisamos calcular a variânça dos campos detectados, definida como $\left\langle(\Delta \hat{I})^{2}\right\rangle=\left\langle\hat{I}^{2}\right\rangle-\langle\hat{I}\rangle^{2}$, de modo que é necessário determinar

$$
\begin{aligned}
& \hat{I}_{+}^{2}=g^{2}\left[\left(\hat{n}_{a}\right)^{2}+\left(\hat{n}_{b}\right)^{2}+2 \hat{n}_{a} \hat{n}_{b}\right], \\
& \hat{I}_{-}^{2}=g^{2}\left[\left(\hat{a}^{\dagger} \hat{b}\right)^{2}+\left(\hat{b}^{\dagger} \hat{a}\right)^{2}+\hat{a}^{\dagger} \hat{a} \hat{b} \hat{b}^{\dagger}+\hat{a} \hat{a}^{\dagger} \hat{b}^{\dagger} \hat{b}\right] .
\end{aligned}
$$

sendo $\hat{n}_{a}$ e $\hat{n}_{b}$ os operadores número de fótons dos feixes $\hat{a}$ e $\hat{b}$, respectivamente. Consideremos $\hat{a}$ como o campo de prova ao qual queremos medir as flutuações e um campo $\hat{b}$ quase-clássico que controla a intensidade e a fase. Portanto, se consideramos o estado do sistema como $|\psi\rangle=\left|\psi_{a}\right\rangle \times|\beta\rangle$ sendo $\left|\psi_{a}\right\rangle$ o estado no modo descrito pelo operador $\hat{a}$ e $|\beta\rangle$ é o estado coerente do sistema de controle $\hat{b}$, obtemos

$$
\begin{aligned}
& \left\langle\hat{I}_{+}\right\rangle=g\left[\left\langle\hat{n}_{a}\right\rangle_{a}+|\beta|^{2}\right] \quad \text { sendo } \quad|\beta|^{2}=\left\langle\hat{n}_{b}\right\rangle_{\beta} \\
& \left\langle\hat{I}_{-}\right\rangle=g\left[\left\langle\hat{a}^{\dagger}\right\rangle_{a} \beta+\langle\hat{a}\rangle_{a} \beta^{*}\right] \\
& \left\langle\hat{I}_{+}^{2}\right\rangle=g^{2}\left[\left\langle\left(\hat{a}^{\dagger} \hat{a}\right)^{2}\right\rangle_{a}+|\beta|^{4}+2\left\langle\hat{n}_{a}\right\rangle_{a}|\beta|^{2}\right] \\
& \left\langle\hat{I}_{-}^{2}\right\rangle=g^{2}\left[\left\langle\left(\hat{a}^{\dagger}\right)^{2}\right\rangle_{a} \beta^{2}+\left\langle\hat{a}^{2}\right\rangle_{a}\left(\beta^{*}\right)^{2}+\left\langle\hat{a}^{\dagger} \hat{a}+\hat{a} \hat{a}^{\dagger}\right\rangle_{a}|\beta|^{2}+\left\langle\hat{n}_{a}\right\rangle_{a}+|\beta|^{2}\right]
\end{aligned}
$$

usando a propriedade de comutação $\left[\hat{b}, \hat{b}^{\dagger}\right]=1$. Pelo fato de que $\left\langle\hat{n}_{a}\right\rangle_{a}=I_{a}=I_{1}+I_{2}$ sendo esta a intensidade média do campo de entrada e considerando $|\beta|^{2}=0$ (vacúo), então finalmente obtemos a variância da soma e da diferença das fotocorrentes:

$$
\begin{aligned}
& \left\langle\left(\Delta \hat{I}_{+}\right)^{2}\right\rangle=g^{2}\left\langle\left(\Delta \hat{n}_{a}\right)^{2}\right\rangle \\
& \left\langle\left(\Delta \hat{I}_{-}\right)^{2}\right\rangle=g^{2} I_{a}
\end{aligned}
$$

em que a subtração é o ruído de Shot-Noise. Este ruído da acesso ao limite de ruído mínimo para uma fonte de luz clássica.

Antes de qualquer medida de ruído, precisamos fazer a calibração de Shot-Noise, para determinar o ruído mínimo do vácuo, estabelecendo assim um referencial com o qual podemos comparar posteriormente o ruído medido quando os campos eletromagnéticos interagem com os átomos. Para isto, devemos levar em conta o fato de que os detetores possuem ganhos um pouco diferentes, o que cria uma desigualdade no cálculo de $\left\langle\left(\Delta \hat{I}_{-}\right)^{2}\right\rangle$. Como explicamos anteriormente, os detectores separam o sinal de baixa frequência (sinal $\mathrm{DC}$ ) e de alta frequência $(\mathrm{HF})$, de modo que podemos representar a corrente fotoelétrica com o modelo linear $\hat{I}=\bar{I}+\delta \hat{I}$, onde $\bar{I}$ corresponde à média da corrente que é medida como sinal DC e $\delta \hat{I}$ corresponde às flutuações desta corrente que é medida através do sinal HF, como é feito em [50], tal que

$$
\begin{aligned}
& \hat{I}_{1}^{\prime}=g \hat{d}^{\dagger} \hat{d} \rightarrow \hat{I}_{1}^{\prime}=g_{1}\left(\bar{I}_{1}+\delta \hat{I}_{1}\right) \\
& \hat{I}_{2}^{\prime}=g \hat{c}^{\dagger} \hat{c} \rightarrow \hat{I}_{2}^{\prime}=g_{2}\left(\bar{I}_{2}+\delta \hat{I}_{2}\right)
\end{aligned}
$$


No entanto devemos considerar que os fotodetetores não só são sensíveis à luz, mas também são sensíveis a flutuações eletrônicas. Portanto devemos considerar que a flutuação medida por cada um dos detetores é

$$
\delta \hat{I}_{i}^{\prime}=g_{i} \delta \hat{I}_{i}+\delta \hat{I}_{\text {elet }_{i}} \quad i=1,2
$$

onde $\delta \hat{I}_{i}$ são as flutuações intrínsecas da luz e $\delta \hat{I}_{\text {elet }_{i}}$ corresponde às flutuações eletrônicas. A partir destas flutuações, podemos calcular o ruído em cada um dos detetores, sendo este a variânça $\Delta^{2} I=\left\langle(\delta I)^{2}\right\rangle$ tal que

$$
\Delta^{2} \hat{I}_{i}^{\prime}=g_{i}^{2} \Delta^{2} \hat{I}_{i}+\Delta^{2} \hat{I}_{\text {elet }_{i}} \quad i=1,2
$$

de modo que a relação entre os ganhos dos detetores $g_{r}=g_{1} / g_{2}$, pode ser determinada com a expressão anterior

$$
g_{r}^{2}=\frac{g_{1}^{2}}{g_{2}^{2}}=\frac{\Delta^{2} \hat{I}_{1}^{\prime}-\Delta^{2} \hat{I}_{\text {elet }_{1}}}{\Delta^{2} \hat{I}_{2}^{\prime}-\Delta^{2} \hat{I}_{\text {elet }_{2}}}
$$

Usando esta relação de ganhos, agora sim podemos determinar a soma e a diferença das fotocorrentes de uma maneira balanceada

$$
\Delta^{2} \hat{I}_{ \pm}^{\prime}=\left\langle\left(\delta I_{1}^{\prime} \pm g_{r} \delta I_{2}^{\prime}\right)^{2}\right\rangle=\Delta^{2} \hat{I}_{1}^{\prime}+g_{r}^{2} \Delta^{2} \hat{I}_{2}^{\prime} \pm 2 g_{r}\left\langle\delta I_{1}^{\prime} \delta I_{2}^{\prime}\right\rangle
$$

Substituindo a expressão (3.31) na equação anterior, e levando em conta o fato de que $\left\langle\delta I_{1}^{\prime} \delta I_{2}^{\prime}\right\rangle=g_{1} g_{2}\left\langle\delta I_{1}^{\prime} \delta I_{2}^{\prime}\right\rangle$ considerando a definição da eq. (3.30) ${ }^{4}$, obtemos

$$
\Delta^{2} \hat{I}_{ \pm}^{\prime}=g_{1}^{2} \Delta^{2} \hat{I}_{ \pm}+\Delta^{2} \hat{I}_{\text {elet }_{1}}+g_{r}^{2} \Delta^{2} \hat{I}_{\text {elet }_{2}}
$$

No caso da diferença, temos que a luz coerente satisfaz $\Delta^{2} \hat{I}_{-}=I_{b}$, onde pela eq.(3.18) temos que $I_{b}=I_{1}+I_{2}$, portanto a calibração de Shot-noise balanceada é

$$
\Delta^{2} \hat{I}_{-}^{\prime}=g_{1}^{2}\left(I_{1}+I_{2}\right)+\Delta^{2} \hat{I}_{\text {elet }_{1}}+g_{r}^{2} \Delta^{2} \hat{I}_{\text {elet }_{2}}
$$

Esta equação estabelece que fazendo o gráfico do ruído da diferença em função da intensidade dos dois detectores, obtemos a constante $g_{1}$ a partir da inclinação da reta de ajuste. Por outro lado, da eq. (3.32) obtemos a constante $g_{r}$ que permite balancear os detectores. Com esta calibração, determinamos o nosso limite de Shotnoise, de modo que durante a experiência com os átomos, vamos dividir todas a medidas de ruído pelo Shot-noise, como ponto de referência.

\footnotetext{
${ }^{4}$ Para provar esta relação, devemos considerar que as flutuações de ruido eletrônico para cada um dos detectores não são correlacionadas, ou seja, $\left\langle\delta I_{\text {elet }_{1}}^{\prime} \delta I_{\text {elet }_{2}}^{\prime}\right\rangle=0$ e do mesmo modo, as flutuações eletrônicas não tem relação com as flutuações da luz, portanto $\left\langle\delta I_{i}^{\prime} \delta I_{\text {elet }_{j}}^{\prime}\right\rangle=0$ sendo $^{\prime}$ $i, j=1,2$.
} 
Neste capítulo descrevemos as diferentes etapas da preparação experimental necessária para fazer a medidas de ruído da luz interagindo com os átomos. Primeramente mostramos as características da nossa nuvem fria de átomos, posteriormente descrevemos o controle de campo magnético necessário, para evitar a quebra de degenerescência que cria dessintonias de frequência, e finalmente introduzimos a calibração de ruído limite Shot-noise, o qual será nosso referêncial nas medidas de ruído no próximo capítulo. 


\section{CAPÍTULO 4}

\section{Espectroscopia de absorção em configuração de EIT de átomos frios de Rubídio 85}

Até agora, mostramos e discutimos de forma teórica o fenômeno de EIT, suas propriedades e possíveis cenários com diferentes estruturas de níveis atômicos. Descrevemos o modelo de transferência de ruído de fase para ruído de amplitude dos campos eletromagnéticos interagindo com o meio atômico, com o qual é possível determinar a correlação induzida na configuração de EIT. Além disso, foi necessário descrever a preperação do meio atômico que é usado no experimento e o esquema de medição do ruído dos campos eletromagnéticos. Descrita a preparação do nosso sistema, neste capítulo mostraremos os resultados experimentais da espectroscopia em configuração de EIT, do sinal médio dos campos (Sinal DC), do ruído dos campos e da correlação entre os campos de prova e controle.

Na primeira parte deste capítulo, mostraremos a montagem experimental e o espectro do nível fundamental $F=2$ para Rb 85, que permitirá determinar a transição atômica que usaremos na experiência de EIT. Na segunda parte mostraremos os espectros de absorção do sinal DC, do ruído e da correlação em configuração de EIT. Analisaremos estes resultados com o modelo teórico de difusão de fase, descrito no capítulo 2. Finalmente mostraremos os espectros destas mesmas variáveis em configuração de Hanle-EIT. 
CAPÍTULO 4. ESPECTROSCOPIA DE ABSORÇÃO EM CONFIGURAÇÃO DE EIT DE ÁTOMOS FRIOS 54

DE RUBÍDIO 85

\subsection{Montagem Experimental}

A figura 4.1 mostra a montagem experimental usada para a experiência de EIT. No experimento usamos lasers de diodo com cavidade externa em configuração Littrow $[51,52]$, cujo comprimento de onda é de $780.24 \mathrm{~nm}$, compatível com a linha D2 do Rubídio. A largura de linha do laser medida é de 1,2 $\pm 0,2 \mathrm{MHz}$. O laser de diodo, usado como laser de prova e de controle é representado pela linha vermelha na figura 4.1. Uma pequena porção da luz é usada para fazer uma espectroscopia de absorção

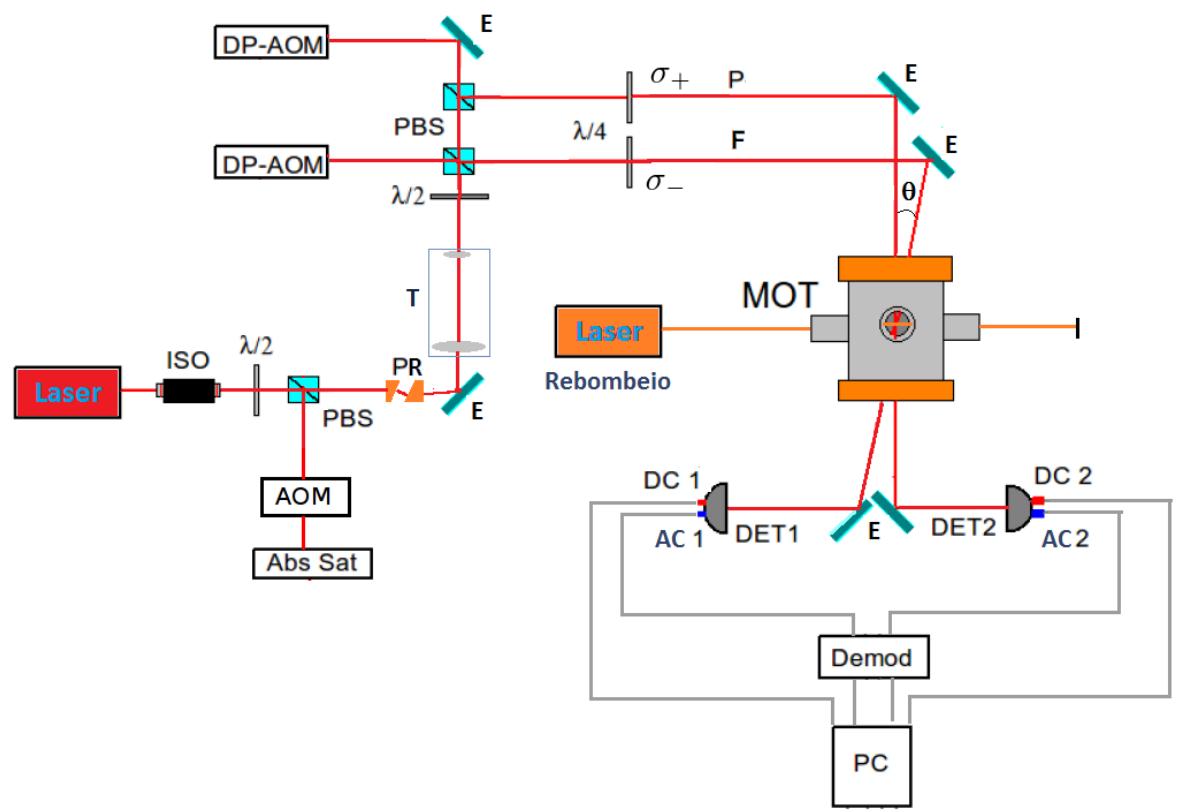

Figura 4.1: Montagem Experimental. ISO: Isolador ótico. $\lambda / 2$ : Lâmina de meia onda. $\lambda / 4$ : Lâmina de quarto de onda. PBS: Polarizer Beam Splitter. AOM: Modulador acusto-ótico. Abs. Sat.:Espectroscopia de absorção Saturada. PR:Prisma anamorfo. E: Espelho. T: Telescópio. DET: Detetor. MOT: Armadilha magnetoótica. Demod: Demodulador. PC:Computador.

saturada [45, 53] em células de vapor de Rubídio. Esta espectroscopia atua como frequência de referência, para sintonizar a frequência do laser que interage com a nuvem de átomos frios numa transição específica.

Dado que o perfil de intensidade transversal do feixe é elíptico na sua saída, usamos dois prismas anamórficos (PR) para corrigi-lo, obtendo um feixe com perfil circular. Depois dos prismas, o feixe passa por um telescópio. A frente de onda que sai do laser possui uma pequena divergência, de modo que o telescópio diminui a cintura do feixe à metade, e corrige a frente de onda, diminuindo a divergência durante o percurso pelos espelhos até a câmara onde estão os átomos frios.

Após passar pelo telescópio, separamos o feixe através de um Polarizer Beam- 
Splitter (PBS), que vai dividir o feixe em componentes de polarização vertical (V) e horizontal (H). Ambos os feixes são acoplados com Moduladores Acusto-Óticos (AOM sigla em inglês), que mediante ondas de Rádio Frequência (RF), modulam a frequência e a amplitude do laser [54]. O fato de manipular a frequência de cada um dos feixes de forma independente, faz com que os feixes possam interagir com os átomos em configuração $\Lambda$ de EIT na transição ${ }^{5} S_{1 / 2} F=2 \rightarrow{ }^{5} P_{3 / 2} F=2$ do $\mathrm{Rb}^{85}$. Usamos um outro laser (linha laranja) como rebombeio, que é o encarregado de transferir a população dos níveis ${ }^{5} S_{1 / 2} F=3$, em que é preparada a nuvem atômica, para o estado ${ }^{5} S_{1 / 2} F=2$. Isto é feito acoplando o laser de rebombeio na transição ${ }^{5} S_{1 / 2} F=3 \rightarrow{ }^{5} P_{3 / 2} F=2$, tal que a emissão espontânea dos átomos faz com que eles decaiam para o estado ${ }^{5} S_{1 / 2} F=2$, sendo este necessário para acoplar os feixes de prova e controle em configuração de EIT.

Os espectros de absorção saturada para os lasers de prova e rebombeio estão respresentados nas figuras 4.2.a e 4.2.b, respectivamente. A figura 4.2.a, mostra o espectro de absorção saturada da transição ${ }^{5} S_{1 / 2} F=2$ para os estados excitados, e a figura 4.2.b, mostra o espectro de absorção saturada da transição ${ }^{5} S_{1 / 2} F=3$ para os estados excitados. Usando o espectro para ${ }^{5} S_{1 / 2} F=2$, estabilizamos a

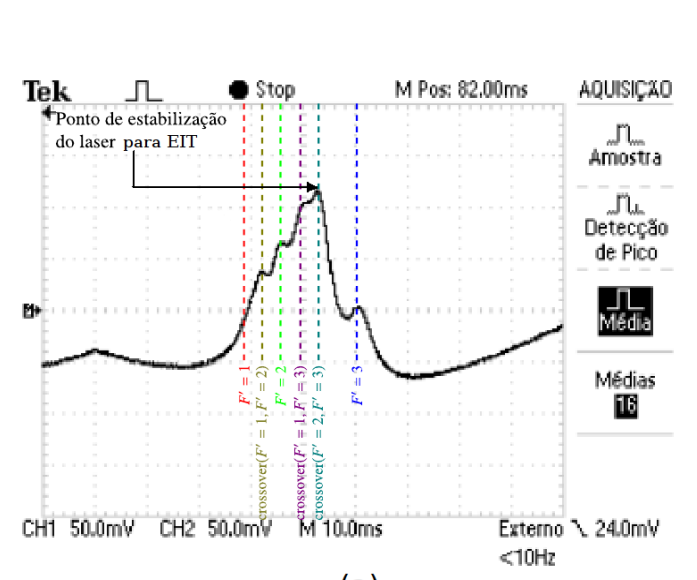

(a)

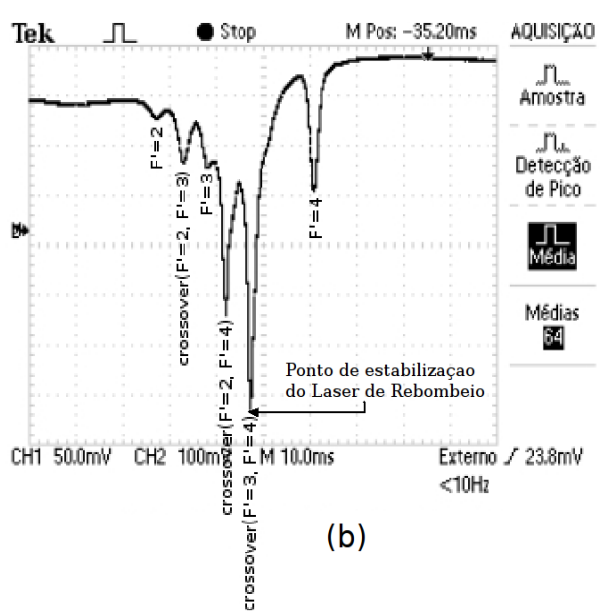

Figura 4.2: Espectroscopia de absorção saturada para (a) a transição ${ }^{5} S_{1 / 2} F=2$ para os estados excitados. (b) a transição ${ }^{5} S_{1 / 2} F=3$ para os estados excitados

frequência do laser de prova, no ponto de cross-over $^{1}\left|F^{\prime}=2, F^{\prime}=3\right\rangle$ em que temos o maior sinal de absorção, e posteriormente deslocamos a frequência para a transição $F=2 \rightarrow F^{\prime}=2$ (mais informação sobre frequência cross-over pode-se encontrar em $[29,30,45,53])$. Da mesma forma, estabilizamos a frequência do laser de rebombeio

\footnotetext{
${ }^{1} \mathrm{~A}$ frequencia de cross-over é a frequência na qual dois grupos de velocidades de átomos absorvem luz mediante efeito Doppler. Isto faz com que o sinal de absorção seja maior que as outras transições. Porém não corresponde a uma transição atômica real.
} 
no ponto de cross-over $\left|F^{\prime}=3, F^{\prime}=4\right\rangle$ do espectro para ${ }^{5} S_{1 / 2} F=3$, e deslocamos a frequência para a transição $F=3 \rightarrow F^{\prime}=2$. A estabilização da frequência do laser é feita mediante o sistema de dither \& lock [53].

Os moduladores acusto-óticos (AOM) permitem deslocar a frequência do laser de maneira eficiente num intervalo de $\pm 20 \mathrm{MHz}$ ao redor de $80 \mathrm{MHz}$, mediante uma rampa de tensão no circuito de controle do AOM ${ }^{2}$. Os AOMs não só criam um deslocamento de frequência, como também espacial, de modo que é necessário implementar um esquema de dupla-passagem, que faz o feixe passsar duas vezes pelo AOM [54]. Isto faz com que o feixe saia na mesma direção de entrada, porém em sentido oposto.

Aplicar este esquema de dupla-passagem, tem a desvantagem de deslocar a frequência do laser duas vezes $(160 \mathrm{MHz})$. Este fato é compensado usando um AOM de passagem simples, para deslocar a frequência do laser em $-100 \mathrm{MHz}$, antes de ocorrer a absorção saturada, de modo que a frequência do laser fica $100 \mathrm{MHz}$ abaixo da frequência original. Nesta condição, o laser passa pelo sistema de AOM em dupla passagem, sintonizando-o na frequência de ressonância para interagir com os átomos frios.

Após a modulação de frequência, os dois feixes com polarizações linear $\mathrm{V}$ e $\mathrm{H}$, passam pelas lâminas de onda $\lambda / 4$, que transformam as polarizações lineares em polarizações circulares opostas $\sigma_{+}$e $\sigma_{-}$. Os dois feixes interagem com a nuvem de átomos num ângulo de $2^{o}$ entre eles. Finalmente são detectados por fotodiodos, em que o sinal DC vai diretamente para aquisição de dados no computador, enquanto o sinal HF é demodulado numa frequência de análise e, posteriormente, os dados são adquiridos no computador. Este esquema de deteção foi descrito na seção 3.5.

Para fazer os espectros de absorção da nuvem, devemos ter um controle sobre o sistema de resfriamento dos átomos e sincronizá-lo com o feixe de prova, para obter os diferentes espectros. Para isto, precisamos ter uma sequência temporal entre os feixes de resfriamento da nuvem e o feixe de prova, como mostra a figura 4.3. Este controle é feito mediante um programa em LabView que controla os dispositivos de tensão que, por sua vez, controlam os moduladores acusto-óticos, que modulam os lasers do sistema (ver interface do programa no apêndice A). Inicialmente carregamos a nuvem de átomos. Depois, num tempo dado, desligamos o campo magnético de aprisionamento e os feixes de resfriamento. Com isto evitamos que o feixe de resfriamento coloque a população atômica em estados não apropriados para fazer a espectroscopia com o feixe de prova. Concomitantemente, desligamos o campo

\footnotetext{
${ }^{2}$ Neste intervalo de varredura de frequência, a eficiência dos AOMs é muito susceptível ao alinhamento do feixe com o AOM, variando a intensidade dos feixes durante a medida. Portanto, usamos intervalos máximo de $\pm 5 \mathrm{MHz}$ de frequência.
} 
magnético, evitando criar uma dessintonia do laser com os níveis atômicos, como foi visto na seção 3.4 .

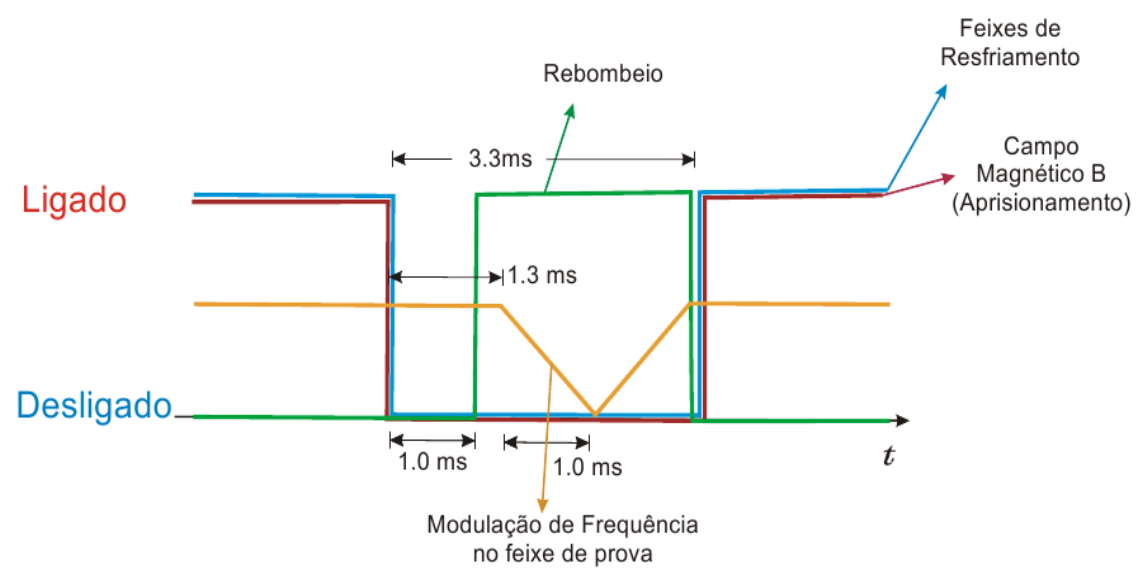

Figura 4.3: Sequência de sinais para fazer a espectroscopia nos átomos frios. A linha azul e marrom, representam a tensão que liga ou desliga os feixes de resfriamento da nuvem, e o campo de aprisionamento. A linha verde representa a tensão do feixe de rebombeio, ligado $1 \mathrm{~ms}$ após desligar o feixe de resfriamento. A linha amarela representa a tensão que modula a frequência do feixe de prova, durante $2 \mathrm{~ms}$

Durante o tempo em que o campo magnético e os feixes de resfriamento são desligados, a nuvem começa a espalhar-se de forma balística, mantendo uma densidade considerável durante 3ms, dentro do qual podemos fazer as medidas de espectroscopia. Depois de esperar 1ms, ligamos o feixe de rebombeio para colocar incialmente a população atômica no estado $F=2$. Posteriormente, esperamos mais $0.3 \mathrm{~ms}$ para fazer a espectroscopia, colocando uma rampa de tensão nos AOMs durante $1 \mathrm{~ms}$ (este é o tempo no qual a rampa de tensão diminui. Porém demoramos mais $1 \mathrm{~ms}$ para aumentar a rampa de tensão, para deixar a frequência dos AOMs como estava inicialmente). No final deste processo, ligamos tudo novamente para carregar a nuvem de átomos.

Dado que os níveis usados para fazer a espectroscopia de absorção são níveis Zeeman, susceptíveis à quebra de degenerescência, a presença de campos magnéticos espúreos, como o campo magnético da terra ou o campo criado pela bomba Iónica do sistema de vácuo da câmara, onde esta a nuvem atômica, precisam ser compensados. O campo de compensação é gerado por um conjunto de bobinas em configuração Helmoltz, colocadas nos três eixos espaciais. Cada uma destas bobinas possui 16 espiras de fio, ligadas numa fonte de corrente, que fornece uma corrente máxima de $I=0.8 \mathrm{~A}$. Com estas bobinas criamos, no seu centro, um campo de compensação $B=0.27$ Gauss aproximadamente ${ }^{3}$.

\footnotetext{
${ }^{3} \mathrm{O}$ campo é estimado a partir da corrente $I=0.201(1)$ A que circula pela bobina.
} 


\subsection{Espectroscopia de absorção da nuvem atômica do estado fundamental $\mathbf{F}=\mathbf{2}$ do $\mathrm{Rb}^{85}$}

No capítulo 1 mostramos vários perfis de absorção de EIT. No laboratório usamos a transmissão do feixe para obter informação de quanta luz foi absorvida pelos átomos. De modo que os espectros mostrados a seguir correspondem à transmissão do laser

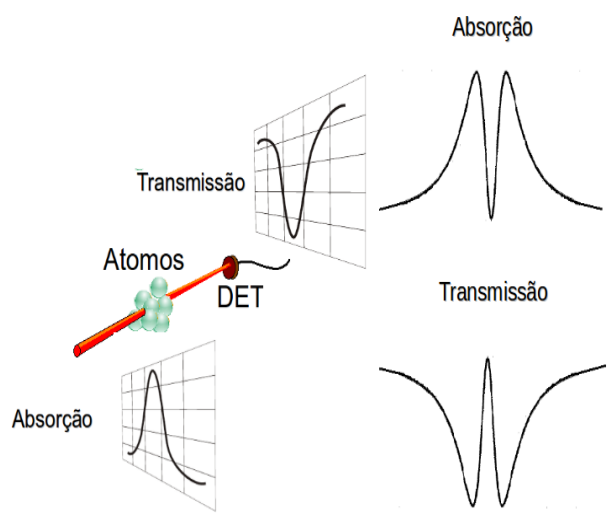

Figura 4.4: Relação entre a absorção atômica e a transmissão do feixe de prova.

de prova, que muda de acordo com a absorção do meio atômico, como mostra a figura 4.4. A transmissão do feixe esta relacionada com a absorção do meio por $T(\omega) \rightarrow \exp \left\{-\beta \Im \mathrm{m}\left[\chi^{(1)}(\omega)\right]\right\}$ onde $\beta$ representa a profundidade ótica $[35,45]$.

Antes de fazer um espectro de EIT numa transição específica, devemos fazer a espectroscopia dos níveis hiperfinos da nuvem atômica, e compará-la à nossa frequência de referência obtida a partir da absorção saturada. Na figura 4.5, mostramos o espectro de absorção da nuvem atômica na transição ${ }^{5} S_{1 / 2} F=2 \rightarrow{ }^{5} P_{3 / 2} F^{\prime}=1,2,3$, para o feixe de prova e controle. Os espectros mostram-se numa escala relativa junto ao espectro de absorção saturada. Para obter este espectro, é necessário sintonizar o laser de rebombeio na transição ${ }^{5} S_{1 / 2} F=3 \rightarrow{ }^{5} P_{3 / 2} F=2$ do $\mathrm{Rb}^{85}$, da forma como foi explicado na seção anterior.

A partir da espectroscopia de absorção da nuvem, podemos configurar os moduladores acusto-óticos para sintonisar a frequência do laser na transição ${ }^{5} S_{1 / 2} F=$ $2 \rightarrow^{5} P_{3 / 2} F=2$, para obter uma espectroscopia de EIT. 


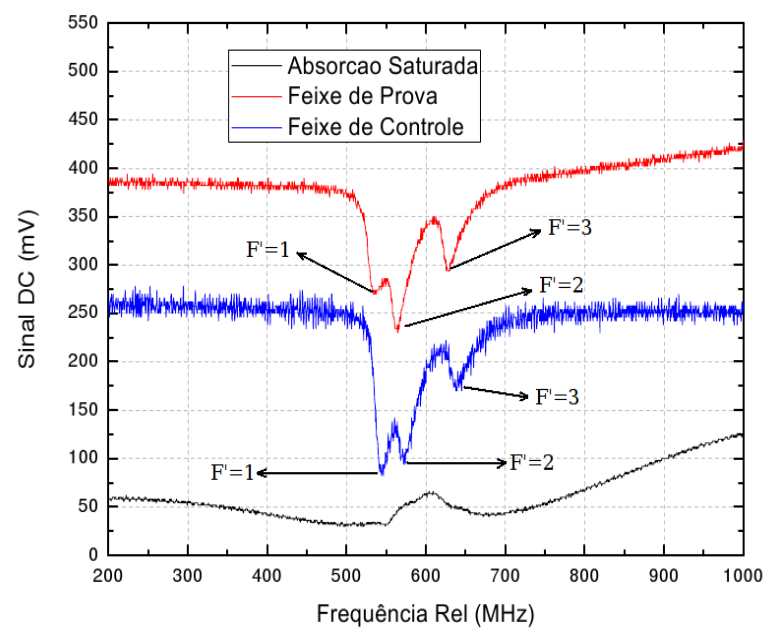

Figura 4.5: Espectroscopia de absorção da nuvem de átomos frios do campo de prova (a linha vermelha) e do campo de controle (a linha azul) na transição ${ }^{5} S_{1 / 2} F=2 \rightarrow{ }^{5} P_{3 / 2} F^{\prime}=1,2,3$. Esta é comparada à espectroscopia de absorção saturada. Estas transições satisfazem as regras de selação $\Delta F=0, \pm 1$.

\subsection{EIT na transição de $F=2$ para $F^{\prime}=2 \mathrm{com}$ átomos frios de $\mathbf{R b}^{85}$}

Dada a preparação dos átomos frios e a estabilização de frequências dos lasers, apresentaremos as medidas de espectroscopia dos átomos frios. Na primeira abordagem das medidas, analisaremos só os espectros de absorção do sinal DC do feixe de prova, para estudar as características do nosso perfil de EIT, e como estas podem mudar segundo as condições de campo magnéticos de aprisionamento. Posteriormente, mostraremos os espectros de ruído e correlação dos campos eletromagnéticos.

Na figura esquerda 4.6, mostramos o espectro de absorção do feixe de prova num intervalo de dessintonia de $\pm 7 \mathrm{MHz}$ sem acoplar o feixe de controle. Este é comparado ao nível de transmissão do feixe de prova quando não temos a presença de átomos na câmara de vácuo. Esta transmissão apresenta uma variação durante a varredura de frequência, devido à eficiência do AOM com o qual fazemos a varredura de frequência.

O espectro de absorção mostra um perfil não muito pronunciado, porque o feixe de prova com polarização $\sigma_{+}$faz um bombeamento ótico para outros níveis Zeeman, tal que perde população que potencialmente poderia ser excitada nessa transição.

Na direita da figura 4.6, mostramos o primeiro espectro com perfil de EIT (que é mais pronunciado, porque o feixe de controle com polarização $\sigma_{-}$traz de volta a população atômica, através de bombeamento ótico, mantendo controlada a população para acoplar o feixe $\sigma_{+}$com os átomos) em que temos $70 \%$ de transparência 

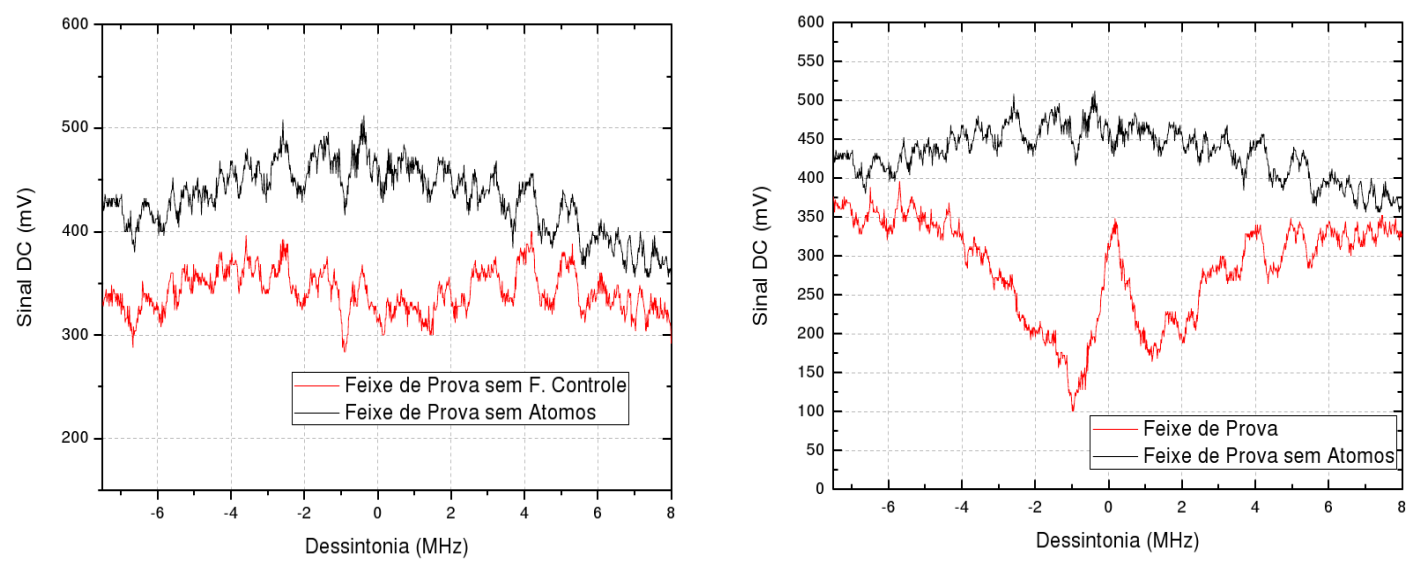

Figura 4.6: (Esquerda) Espectro de absorção do feixe de prova, sem a presença do feixe de controle, sintonizando o laser na transição $F=2$ para $F^{\prime}=2$, modulando a frequência mediante o AOM. (Direita) Espectro de absorção do feixe de prova, em presença do feixe de controle, obtendo EIT.

na condição Raman $\delta_{R}=0$. Esta transparência muda durante cada experiência de medida, dependendo do alinhamento e do número de átomos. Para este perfil de EIT, fazemos um ajuste lorentziano para o pico de transparência, o que permite determinar que a largura de linha $\Gamma_{E I T}=0.72(2) \mathrm{MHz}$. Este valor está em concordância com o valor obtido em [55], para EIT em átomos frios com níveis hiperfinos degenerados.

Para ver as consequências da presença do campo magnético de aprisionamento no perfil de EIT, as figuras 4.7 da esquerda e da direita mostram os espectros de
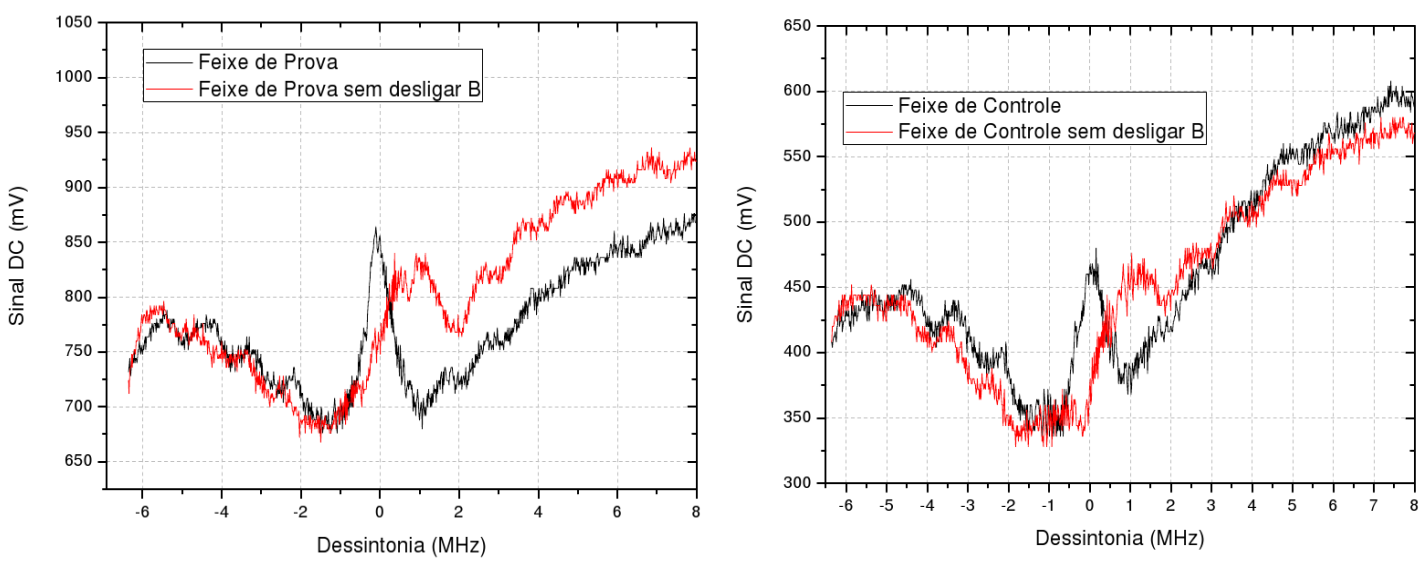

Figura 4.7: (Esquerda) Comparamos o espectro de EIT para o feixe de prova sem desligar (linha preta) e desligando (linha vermelha) o campo de aprisionamento. (Direita) Mesma comparação para o espectro do feixe de controle.

absorção de EIT nesta condição, para os feixes de prova e de controle, respectiva- 
mente (linha vermelha). Estes são comparados ao caso inicial, em que desligamos o campo magnético de aprisionamento da MOT (linha preta).

A presença de campo magnético mostra um deslocamento da frequência na condição Raman (a frequência central do perfil de absorção) pela quebra de degenerescência dos níveis Zeeman, devido ao campo magnético. Além disso, podemos observar que o pico de EIT alarga um pouco, porque muda o ponto de compensação, calibrado anteriormente pelas bobinas de compensação. Isto faz com que a largura de linha do pico de EIT aumente a $\Gamma_{E I T}=1.80(5) \mathrm{MHz}$, em relação ao caso onde não há presença de campo magnético.

\subsection{Espectroscopia de ruído e correlação na con- figuração de EIT com átomos frios}

Para a espectroscopia de ruído é importante levar em conta a relação entre os ganhos dos detectores e a calibração de Shot-noise. Esta última é a fase inicial das medidas de espectroscopia de ruído, que determina o nível de ruído mínimo dos feixes de prova e de controle. A figura 4.8. a mostra o ruído do feixe de controle (DET2) em função do ruído do feixe de prova (DET1). Fazendo o ajuste linear vemos que $\Delta^{2} I_{2}=1.021(2) \Delta^{2} I_{1}$, o que é proximo à unidade, mostrando que os detectores são quase iguais, como se esperaria idealmente. No entanto devemos corrigir esta pequena diferença, como foi descrito na seção 3.5 .
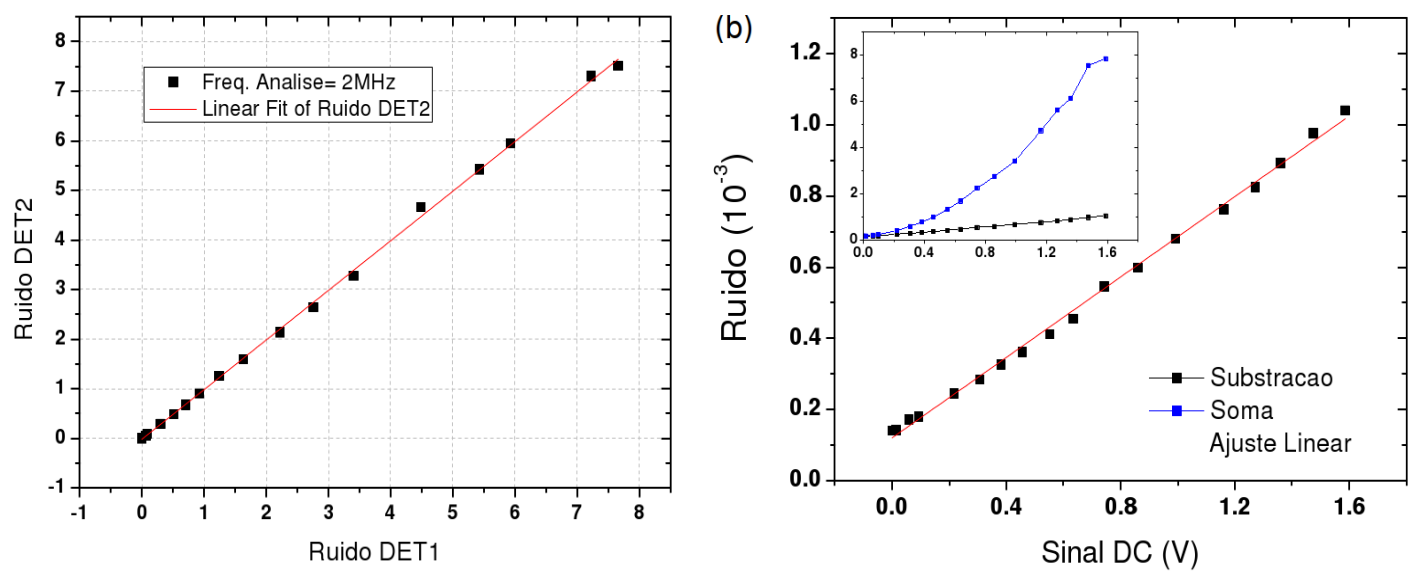

Figura 4.8: Na frequência de análise de $2 \mathrm{MHz}$, mostramos (a) Ruído do feixe de controle em função do ruído do feixe de prova. (b) Subtração dos ruído em função do sinal DC (cuja relação com potência é $P / V=205.6(5) \mu \mathrm{W} / \mathrm{V}$ ), proporcional a potências dos feixes. Isto corresponde a calibração de Shot-noise. No gráfico interno comparamos a subtração com a soma dos ruídos.

Do outro lado, a figura 4.8.b mostra o comportamento linear da subtração dos 
ruídos, de modo que para cada valor de intensidade, temos o valor de ruído mínimo na frequência de análise de $2 \mathrm{MHz}$. Todos os valores de ruído que foram medidos durante a experiência com os átomos, foram normalizados pelo valor de Shot-noise. No gráfico interno, mostramos a soma e a diferença dos ruídos, em que a soma apresenta exceso de ruído, porque acumula os ruídos próprios do laser.

Na tabela 4.1 mostramos os diferentes valores de potência usados nos feixes de prova e de controle para a espectroscopia de EIT. A diferença de potência entre os dois feixes é de $7 \%$ a 10\%. Fizemos medidas para 6 valores de potência diferentes.

\begin{tabular}{c|c}
\hline \hline F. Prova $(\mu \mathrm{W})$ & F. Controle $(\mu \mathrm{W})$ \\
\hline 42 & 39 \\
79 & 70 \\
115 & 106 \\
142 & 131 \\
205 & 199 \\
315 & 292 \\
\hline \hline
\end{tabular}

Tabela 4.1: Potências usadas no feixe de prova e de controle.

Agora analisamos os espectros de absorção, de ruído e de correlação da nuvem atômica. Para uma potência de $P=42 \mu \mathrm{W}$ do feixe de prova e de $P=39 \mu \mathrm{W}$ do feixe de controle, mostramos os espectros de absorção e de ruído, na figura 4.9. Nos gráficos $a$ e $b$ da figura 4.9, temos os espectros de absorção do sinal DC, em que a largura a meia altura do pico de EIT do feixe de prova e controle é de 0.61(1)MHz e $0.528(5) \mathrm{MHz}$,respectivamente. Os espectros de ruído aparecem nos gráficos $c$ e $d$.

Pode-se visualizar o modelo de transformação do ruído de fase para o ruído de amplitude, como a flutuação de intensidade no perfil de absorção (ruído de amplitude), devido a uma pequena flutuação $\Delta \omega$ (ruído de fase). De modo que, na ressonância, uma flutuação $\Delta \omega$ tem uma flutuação quase nula na amplitude. Mapeando esta análise do espectro de ruído na ressonância, este é mínimo. Agora, se analisarmos o ponto a meia altura do pico de absorção de EIT, a flutação de fase levará uma maior flutuação de amplitude. A flutuação de fase no ponto de maior absorção também gera uma flutuação mínima de amplitude. Portanto, podemos associar ao espectro de ruído, a derivada ao quadrado do espectro de absorção.

A figura 4.10.a mostra a correlação dos feixes sem presença dos átomos, o que mostra uma correlação nula e a figura 4.10.b, mostra a correlação dos feixes quando interagem com a nuvem atômica. Podemos observar que na condição Raman temos a máxima correlação, e ao redor da ressonância, os átomos geram uma anti-correlação 

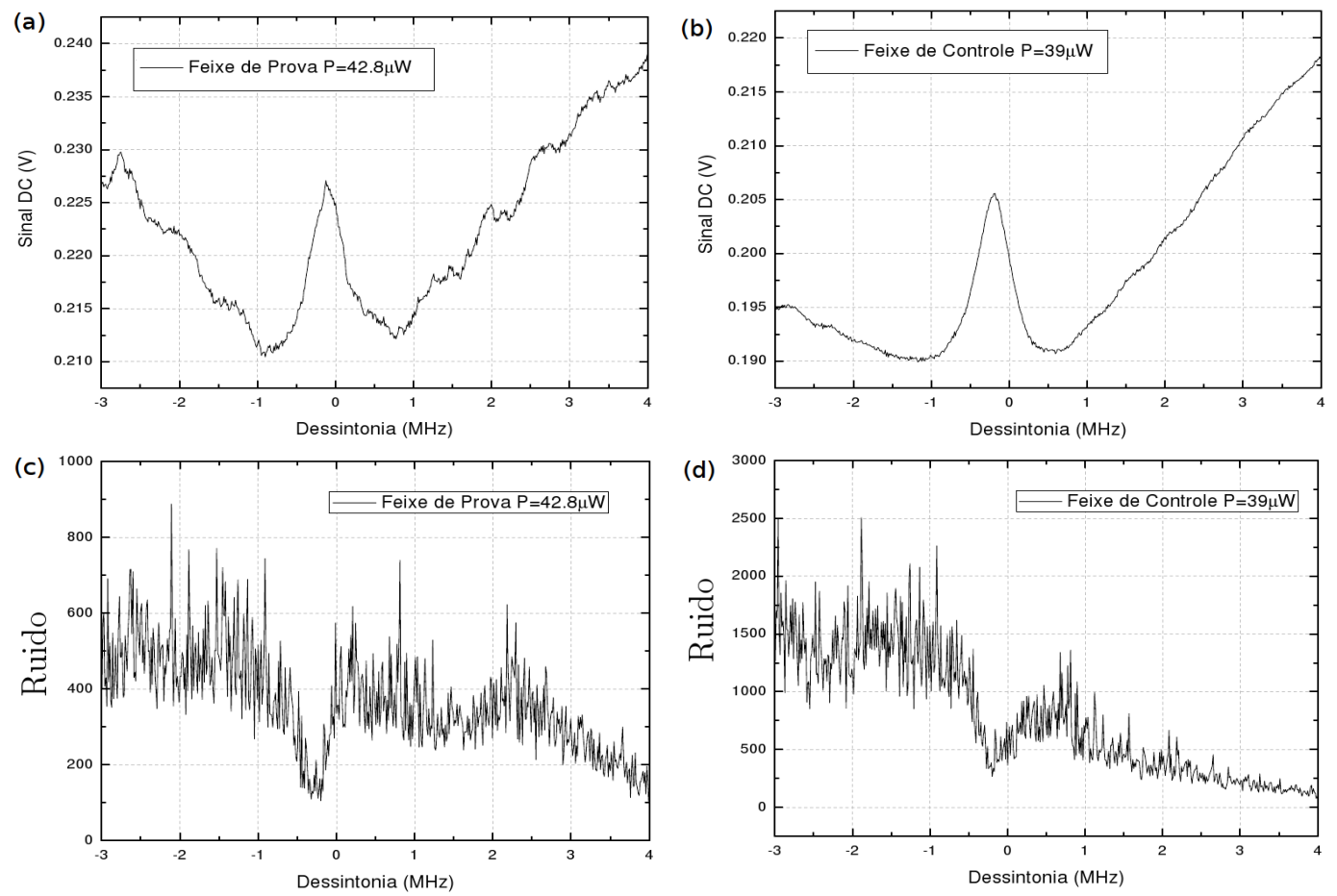

Figura 4.9: (a) e (b) Espectro de absorção do sinal DC para o feixe de prova e de controle, respectivamente. (c) e (d) Espectro de ruído para o feixe de prova e controle, respectivamente.

nos feixes, sem chegar a anti-correlacionar completamente os feixes. O comportamento dispersivo da correlação ao redor da ressonância, está dentro da janela de transparência do espectro de absorção do sinal DC. A máxima absorção do sinal DC
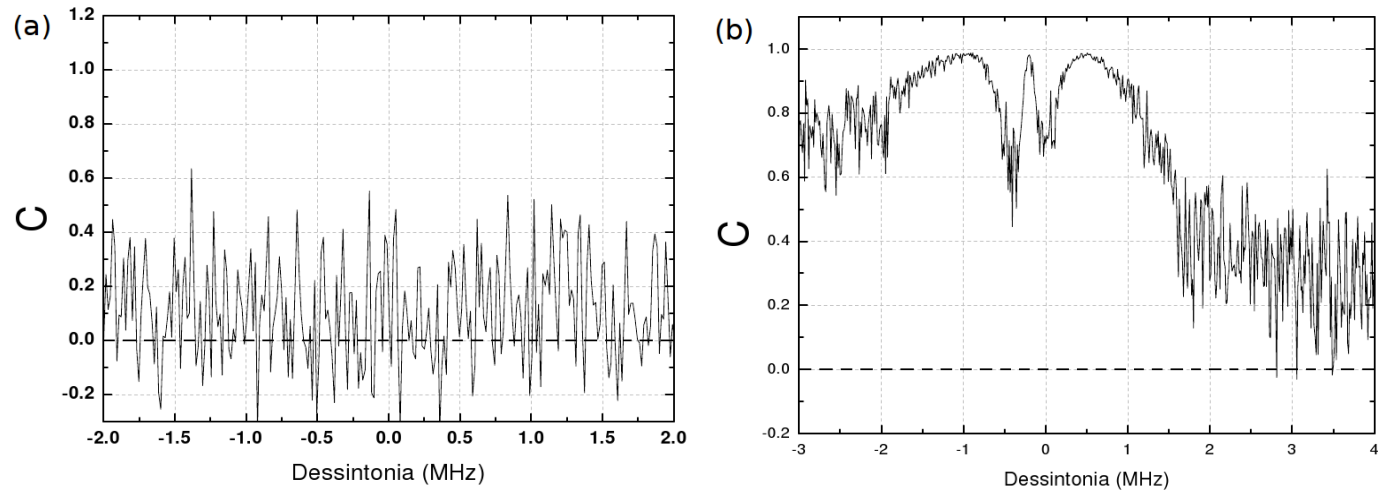

Figura 4.10: (a) Espectro de correlação sem presença dos atomos frios. (b) Espectro de correlação em presença dos átomos frios, para uma potencia $P=42 \mu \mathrm{W}$ e $P=39 \mu \mathrm{W}$, para o campo de prova e controle, respectivamente.

ocorre para uma dessintonia do campo de prova de $\pm 1 \mathrm{MHz}$, obtendo novamente a 
CAPÍTUlO 4. ESPECTROSCOPIA DE ABSORÇÃO EM CONFIGURAÇÃO DE EIT DE ÁtOMOS FRIOS 64 DE RUBÍDIO 85

máxima correlação dos campos. Fora desta janela, a correlação diminui até anularse. A largura do pico de correlação próximo à ressonância é de $0.14(3) \mathrm{MHz}$, menor que a largura do sinal DC.

Para estudar o que acontece com a largura dos picos de EIT do sinal DC e da correlação à medida que aumentamos a potência dos feixes, fizemos uma espectrocopia para os valores de potência da tabela 4.1. A figura 4.11.a mostra os perfis de absorção em EIT do sinal DC, e a figura 4.11.b mostra o espectro de ruído, para as potências: $P=42,79,115,142,205,315 \mu \mathrm{W}$, do feixe de prova. As figuras 4.12.a e
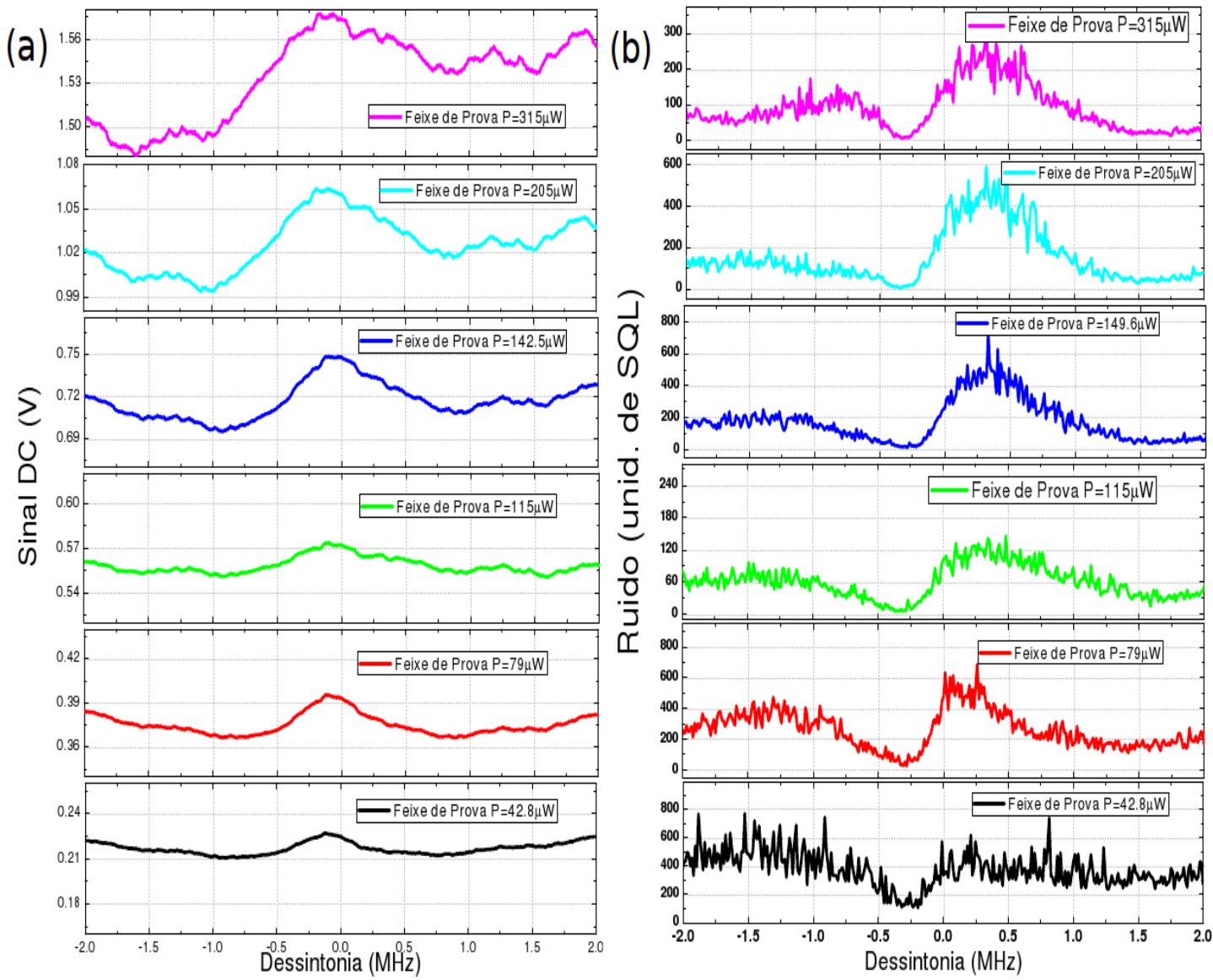

Figura 4.11: (a) Espectro de absorção para o sinal DC do feixe de prova, num intervalo de $\pm 2 \mathrm{MHz}$ de dessintonia, para as potências $P=42,79,115,142,205,315 \mu \mathrm{W}$. (b) Espectro de ruído para os respectivos espectros de absorção do sinal DC, do feixe de prova.

4.12.b mostram o espectro de absorção do sinal DC e o espectro de ruído, respectivamente, para as potências: $P=39,70,106,131,199,292 \mu \mathrm{W}$ do feixe de controle. Os espectros de absorção do sinal DC, conforme aumentamos a potência dos feixes, mostram de fato o alargamento do pico de EIT do sinal DC. 

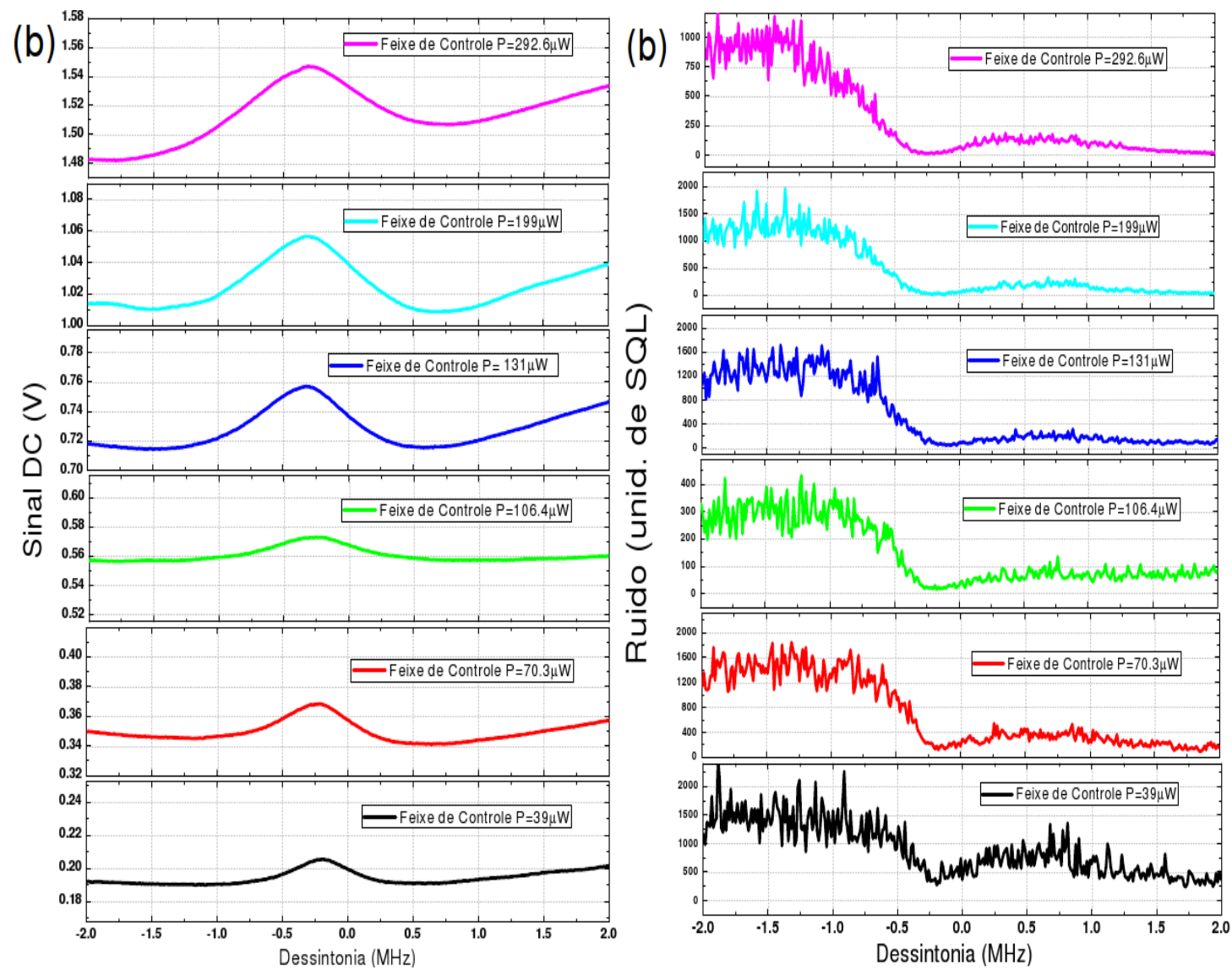

Figura 4.12: (a) Espectro de absorção para o sinal DC do feixe de controle, num intervalo de $\pm 2 \mathrm{MHz}$ de dessintonia, para as potências $P=39,70,106,131,199,292 \mu \mathrm{W}$. (b) Espectro de ruído para o respectivo espectro de absorção do sinal DC do feixe de controle.

Na seção 1.4 discutimos brevemente, no caso em que consideramos o feixe de controle mais intenso do que o feixe de prova, o alargamento por potência do perfil de absorção obtidos de forma teórica, mostrando que mantém concordância com estes resultados experimentais. No entanto, no caso experimental, temos que os dois feixes possuem uma intensidade muito próxima. Para isso, analisaremos em breve, os resultados teóricos a partir do modelo descrito no capítulo 2, com o qual podemos considerar os dois campos com intensidades iguais, e levar em conta as propriedades de excesso de ruído dos campos eletromagnéticos.

A partir dos espectros de ruído de cada um dos feixes $S_{\text {Prova }}$ e $S_{\text {Cont }}$, e a correlação cruzada $S_{P C}$, obtemos a correlação $C=S_{P C} / \sqrt{S_{\text {Prova }} S_{\text {Contr }}}$ da figura 4.13 , para os dois grupos de potência usados na experiência.

As linhas preta e vermelha das figuras 4.13, são a correlação que existe entre o feixe de prova e de controle para baixas potências. Esta correlação indica que, o 

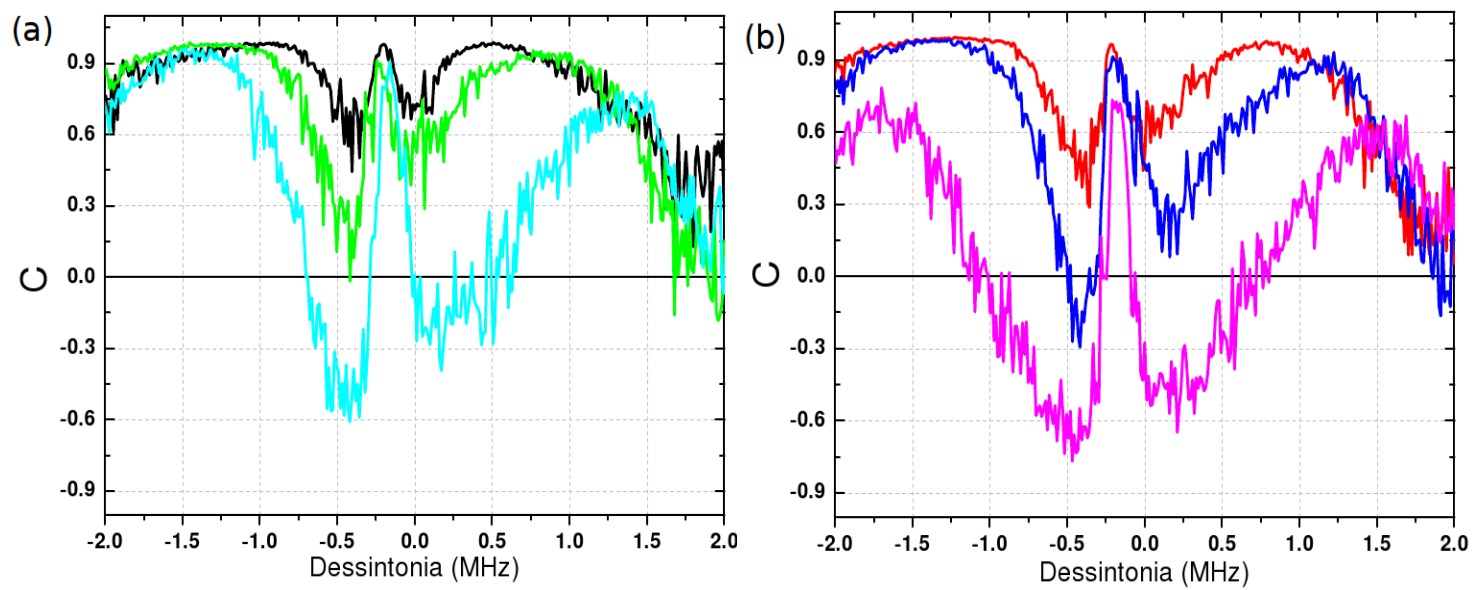

Figura 4.13: (a) Espectro de correlação para potências do campo de prova $P_{P}$ e de controle $P_{C}: P_{P}=42 \mu \mathrm{W}$ e $P_{C}=39 \mu \mathrm{W}$ (Linha preta), $P_{P}=115 \mu \mathrm{W}$ e $P_{C}=106 \mu \mathrm{W}$ (Linha verde) e $P_{P}=205 \mu \mathrm{W}$ e $P_{C}=199 \mu \mathrm{W}$ (Linha azul clara). (b) $P_{P}=79 \mu \mathrm{W}$ e $P_{C}=70 \mu \mathrm{W}$ (Linha vermelha), $P_{P}=142 \mu \mathrm{W}$ e $P_{C}=131 \mu \mathrm{W}$ (Linha azul) e $P_{P}=315 \mu \mathrm{W}$ e $P_{C}=292 \mu \mathrm{W}$ (Linha rosa).

aumento na flutuação de um campo traz como consequência o aumento na flutuação do outro campo. No entanto, conforme aumentamos a intensidade dos campos nas regiões longe da ressonância, os campos passam a um regime de anti-correlação, representadas pelas linhas azul e verde. A anti-correlação implica no aumento da flutuação de um campo, influenciando a diminuição nas flutuações do outro campo. Este fenômeno foi tratado em [31, 33].

O espectro de correlação mostra que, quando os campos satisfazem a condição Raman $\delta_{R}=0$, possuem a máxima correlação. Isto acontece porque o meio atômico é transparante para os campos, de modo que um campo flutuando induz transparência no outro, fazendo com que este não seja absorvido. À medida que aumentamos a intensidade do campo, mantem-se a condição de transparência e, portanto, a flutuação no outro campo também aumenta, como mostra a figura 4.14.a. Podemos observar que embora a intensidade aumente, na condição Raman, sempre atinge o ponto de máxima correlação.

A figura 4.14.b mostra que, quando o campo de prova sai da condição Raman, para uma potência baixa, a correlação dos campos diminui, no entanto, se mantém possitiva. Isto é devido à transição de um fóton, dado que um campo é ressonante com o nível atômico, enquanto o outro não. Isto faz com que o aumento na flutuação de intensidade de um campo, induz o aumento na flutuação de intensidade do outro, em diferente proporção.

No caso em que a intensidade aumenta, quando o campo de prova sai da condição Raman, a correlação dos campos se torna anti-correlação, devido à transição de 
(a)

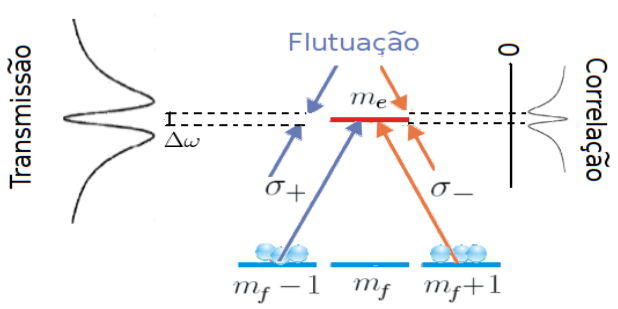

(b)

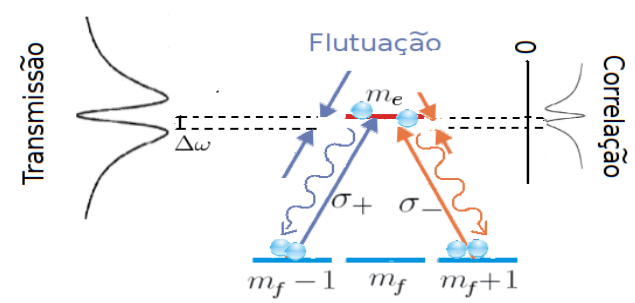

(c)

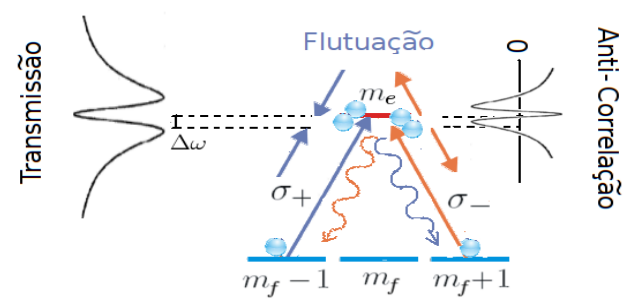

Figura 4.14: Esquema de correlação dos campos. (a) Os campos satisfazam a condição Raman, de modo que possuem a máxima correlação (as flechas possuem o mesmo tamanho e a mesma direção). Isto acontece por ser o ponto de máxima transparência. Este máximo de absorção antige para qualquer valor de intensidade dos campos. (b) A correlação diminui, devido à dessintonia de um dos campos, mas se mantém positiva. Por ter uma baixa potência, os campos induzem transições de um fóton, criando uma flutuação em diferente proporção (as flechas possuem diferentes tamanhos, mas na mesma direção). (c) Para o caso em que a potência dos campos aumenta, estes se tornam anti-correlacionados, devido a dessintonia de um dos campos. Neste caso, os campos induzem transições Stokes (as flechas possuem o mesmo tamanho, mas direções opostas).

Stokes, como mostra a figura 4.14.c. Nesse caso, a absorção de um fóton num campo, diminui a flutuação nele, enquanto a emissão do fóton absorvido, aumenta a flutuação do outro campo. O fato da largura do pico de correlação não alargar está ligada com a condição Raman. Enquanto os campos não estejam muito próximos da condição Raman para obter o máximo de correleção, os campos vão ter estes dois efeitos, que diminui a correlaçao e se torna anticorrelação, para uma dessintonia $\Delta \omega$. A seguir, veremos que esta largura $\Delta \omega$ muda muito pouco à medida que aumentamos a intensidade.

Para cada um dos picos de correlação próximos da ressonância, fizemos o ajuste lorentziano para determinar a largura a meia altura do pico. A figura 4.15 mostra a largura do pico de EIT do sinal DC e da correlação em função da intensidade. Para determinar a intensidade dos feixes medimos a área de cada um dos feixes Gaussianos, obtendo $A_{p}=0.38 \mathrm{~cm}^{2}$ e $A_{c}=0.18 \mathrm{~cm}^{2}$, a partir da cintura de cada um dos feixes.

O pico de EIT do sinal DC apresenta alargamento por potência enquanto o pico de correlação possui menor alargamento em proporção ao sinal DC. O comportamento assintótico da largura do sinal DC, permite pensar que para potências muito baixas, esta largura converge a largura de correlação. O efeito de livre alargamento por potência para a correlação já foi reportado por Xiao et al em [34], para células de vapor (átomos quentes), porém foi brevemente discutido. É importante ressaltar, 
CAPÍTULO 4. ESPECTROSCOPIA DE ABSORÇÃO EM CONFIGURAÇÃO DE EIT DE ÁTOMOS FRIOS
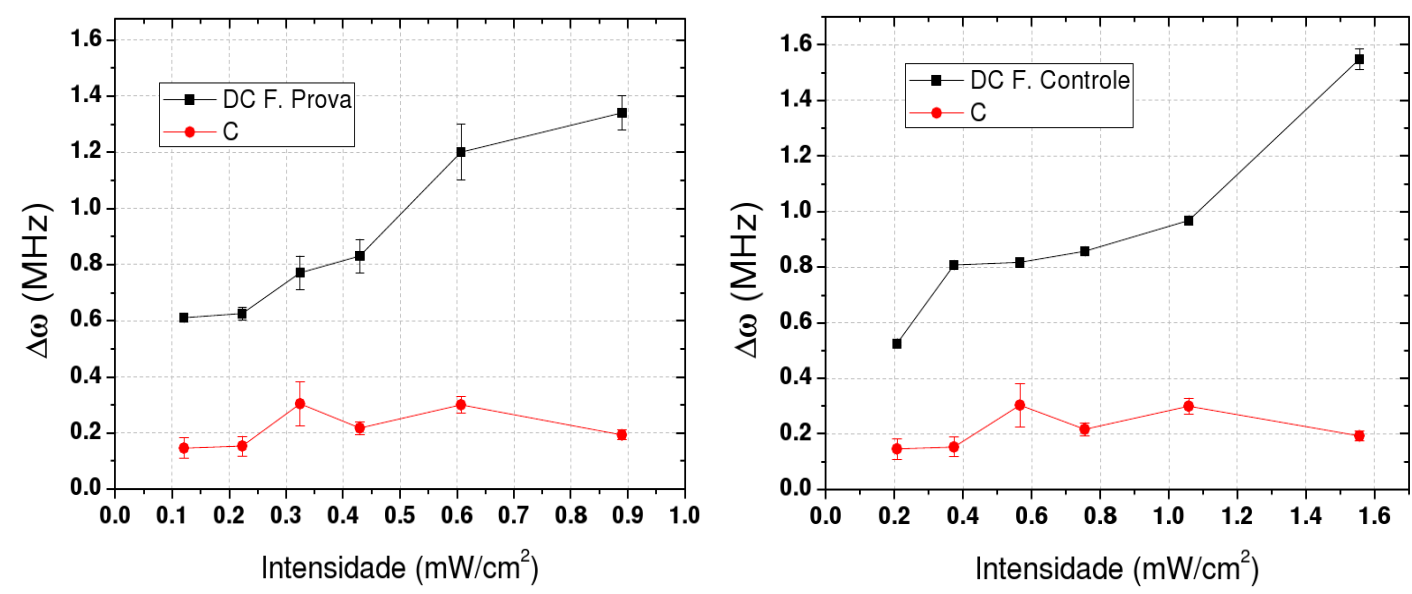

Figura 4.15: Largura do pico de EIT para o sinal DC (Linha preta) e da correlação (Linha vermelha), para (a) Feixe de prova e (b) Feixe de controle.

que nossos resultados foram obtidos para átomos frios.

A seguir apresentaremos os resultados numéricos obtidos para os espectros do valor médio do campo, do ruído e da correlação, mediante o modelo descrito no capítulo 2. Para uma potência dada, usando a solução do vetor $x$ da eq.(2.23), obtemos a coerência do feixe de prova que está relacionado com a absorção do feixe. É possível comparar o espectro desta coerência com o espectro de absorção do sinal DC dos dados experimentais. O espectro de ruído de cada um dos feixes $S_{11}(\omega)$ (relacionada com o espectro do feixe de prova $S_{P}$ ) e $S_{22}(\omega)$ (relacionada com o espectro do feixe de controle $S_{C}$ ) são calculados a partir da solução da eq.(2.40). A correlação, é calculada juntamente com a solução da eq.(2.40), numa frequência de análise de $2 \mathrm{MHz}$, usada na nossa experiência.

As figuras 4.16.a e 4.16.b mostram os espectros da coerência $\rho_{13}$ e do ruído $S_{11}$, para o feixe de prova com diferentes valores da frequência de Rabi. Estas frequências mantém a mesma proporção das intensidades dos feixes usadas no experimento. Observa-se o fenômeno de alargamento por potência visto nos resultados experimentais.

Por outro lado, temos a correlação dos feixes na figura 4.17.a, que reproduz os perfis de correlação obtidos no experimento, ou seja, vemos que ao redor da ressonância passamos de correlação para anti-correlação, à medida que aumentamos a potência dos feixes. Além disso, confirmamos que esta variável do sistema é livre de alargamento por potência. A figura 4.17.b, mostra a largura do pico de EIT da coerência e da correlação, o que está de acordo qualitativamente com os resultados experimentais. 

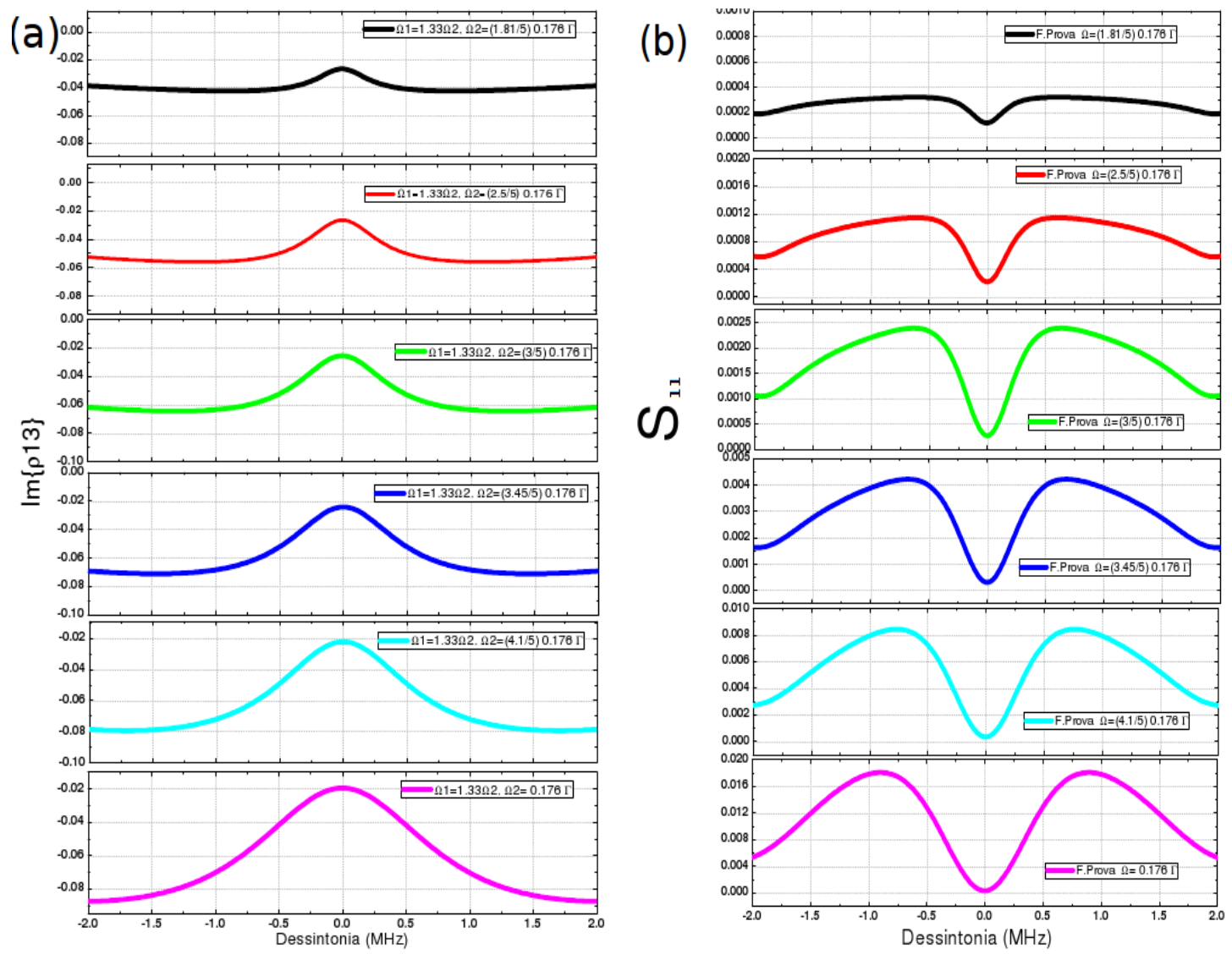

Figura 4.16: (a) Espectro de absorção da coerência $\rho_{13}$ do campo de prova, com parametro de ajuste $\Omega_{0}=0.176 \Gamma / 5$ que concorda com os dados experimentais, para frequências de Rabi $\Omega=1.8 \Omega_{0}$ (Linha preta), $\Omega=2.5 \Omega_{0}$ (Linha vermelha), $\Omega=3 \Omega_{0}$ (Linha verde), $\Omega=3.5 \Omega_{0}$ (Linha azul), $\Omega=4.1 \Omega_{0}$ (Linha azul claro), $\Omega=$ $5 \Omega_{0}$ (Linha Roxa). Este valores mantém a proporção relativa aos valores de intensidade usadas no experimento.(b) Espectro do ruído $S_{11}$ do feixe prova para as mesmas frequências de Rabi.

O alargamento por potência do sinal DC é devido ao fenômeno de saturação do meio, à medida que aumentamos a intensidade, da mesma forma como acontece em qualquer perfil de absorção [39, 40, 45, 53]. No entanto, a correlação é uma quantidade que não pode ser explicada como um fenômeno de saturação, mas como um fenômeno que envolve a condição Raman, as transições de um foton e as transições de Stokes.

Uma experiência semelhante foi feita no laboratorio de Átomos frios do departamento de Fisica da Universidade Federal de Pernambuco (UFPE), pelo Prof. Daniel Felinto, Prof. J.W. Tabosa e Prof. Luciano Cruz (colaborador da UFABC), com o qual o LMCAL tem colaboração [56]. Nesta experiência foram usados átomos frios de Césio, acoplando o feixe de prova e controle à transição entre os níveis hiperfinos $F=3$ e $F=2$, obtendo a espectroscopia de correlação da figura 4.18. Embora a 

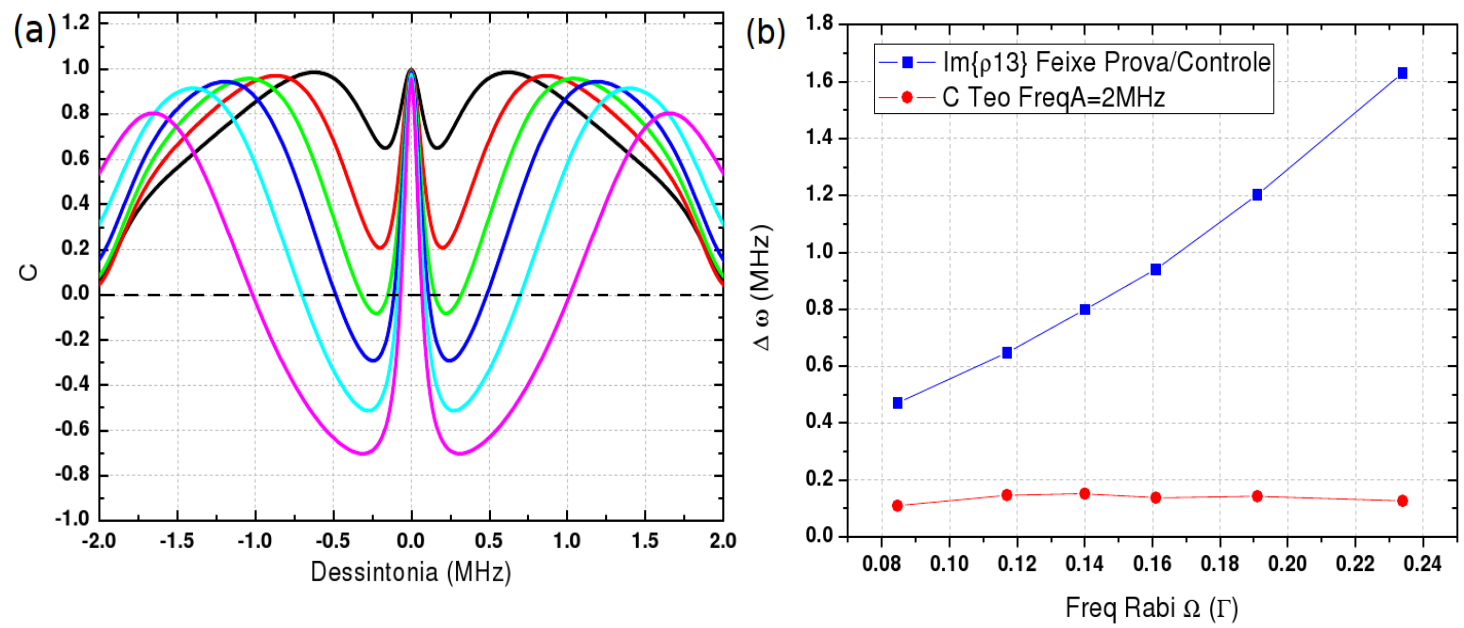

Figura 4.17: (a) Espectro de correlação teórica, com frequências de Rabi $\Omega=(1.8 / 5) 0.176 \Gamma$ (Linha preta), (2.5/5)0.176Г(Linha vermelha), (3/5)0.176Г(Linha verde), (3.5/5)0.176Г(Linha azul), (4.1/5)0.176Г(Linha azul claro), $0.176 \Gamma$ (Linha Rosa).(b) Largura do pico de EIT da coerência $\rho_{13}$ (Linha azul ) e da correlação (Linha vermelha), dos espectros teóricos, em função da frequência de Rabi

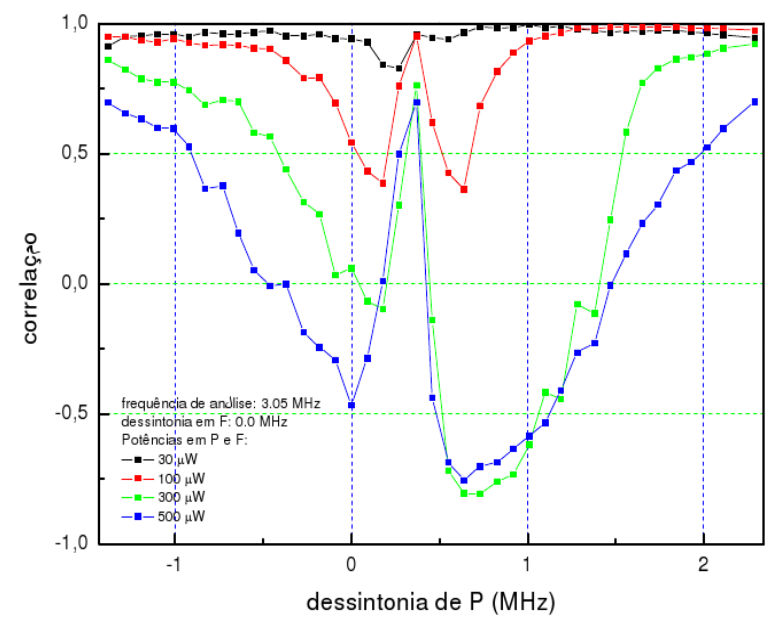

Figura 4.18: Espectro de correlação do Césio para potência do feixe de prova e controle $P=$ $30,100,300,500 \mu \mathrm{W}$.

transição atômica usada possua um maior grau de degenerescência (12 níveis) em relação à transição usada neste trabalho (10 níveis), os espectros apresentam as mesmas características, em forma e escala de frequência.

Com a espectroscopia de correlação da figura 4.13 e da figura 4.18, mostramos que, independentemente da especie atômica, a correlação é livre de alargamento por potência. Inclusive, o não-alargamento do pico de correlação em configuração de EIT, será reportado pelos dois grupos. 
Além da experiência de EIT modelada por um sistema de três níveis, estudamos brevemente o que acontece quando aplicamos campos magnéticos para obter o fenômeno de Hanle-EIT. Na próxima seção mostraremos os espectros de absorção do sinal DC, do ruído e da correlação, com o efeito de Hanle-EIT.

\subsection{Espectroscopia de absorção, de ruído e da cor- relação na condição de Hanle-EIT}

O efeito Hanle acontece por exemplo, quando um feixe de luz, com polarização linear (que pode ser vista como uma superposição de campos com polarização circular $\sigma_{+}$ e $\sigma_{-}$) interage com átomos, em presença de um campo magnético. Este campo quebra a degenerescência dos níveis Zeeman, fazendo com que os átomos absorvam as componentes $\sigma_{+}$e $\sigma_{-}$, de forma diferente. Quando o campo magnético é nulo, obtemos EIT para as duas componentes de polarização circular.

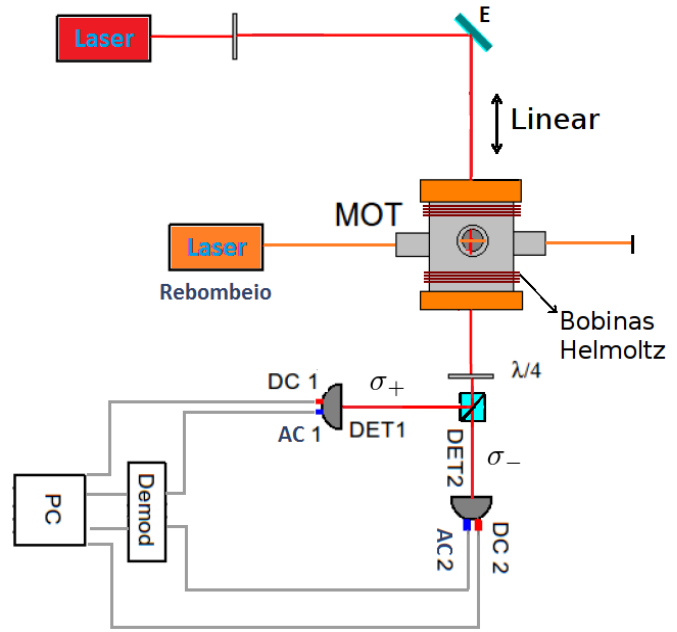

(a)

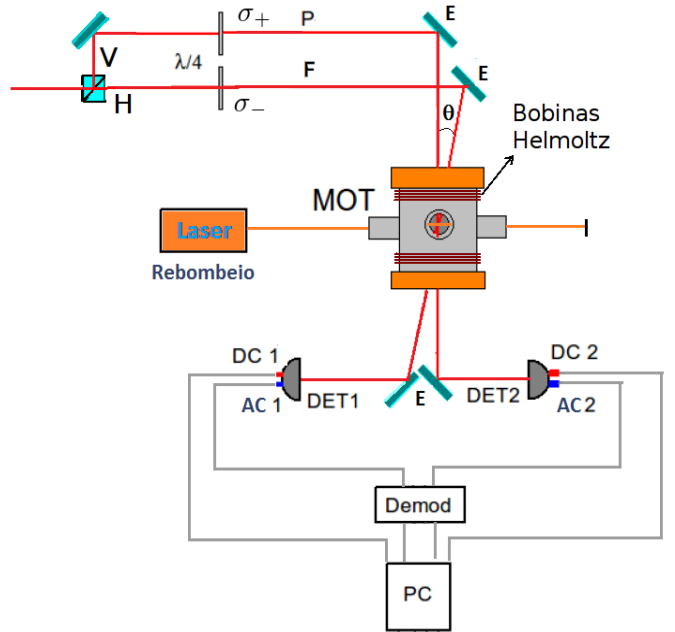

(b)

Figura 4.19: (a) Montagem experimental na experiência de Hanle-EIT com campo eletromagnetico com polarização linear interagindo com os átomos (b) Experiência de Hanle-EIT separando as componentes $\sigma_{ \pm}$antes de interagir com os átomos

Experimentalmente, é necessário gerar um campo magnético maior do que 1 Gauss aproximadamente, o que em frequência corresponde a uma dessintonia de $0.5 \mathrm{MHz}$ ou $1 \mathrm{MHz}$ dos níveis fundamentais $|m f=1\rangle$ ou $|m f=2\rangle$, respectivamente. Estes valores de dessintonia não permitem obter um espectro resolvido. No entanto, no começo do experimento, as bobinas (em configuração Helmoltz) de compensação criavam justamente um campo de 1 Gauss, aproximadamente (o necessário para 
funcionar como um campo de compensação), que não permitia quebrar a degenerescência dos estados Zeeman para obter um espectro de absorção com certo grau de resolução .

Para resolver esta limitação, foi necessário mudar a fonte de corrente das bobinas para uma fonte que fornece uma corrente de $5 \mathrm{~A}$. Fazendo as modificações no circuito, colocando componentes ressistentes a esta corrente, conseguimos fazer variações da corrente num intervalo de $\pm 5 \mathrm{~A}$. Isto gera uma variação máxima de \pm 6.8 Gauss no campo magnético produzido pelas bobinas de compensação no eixo $\hat{z}$. No entanto,

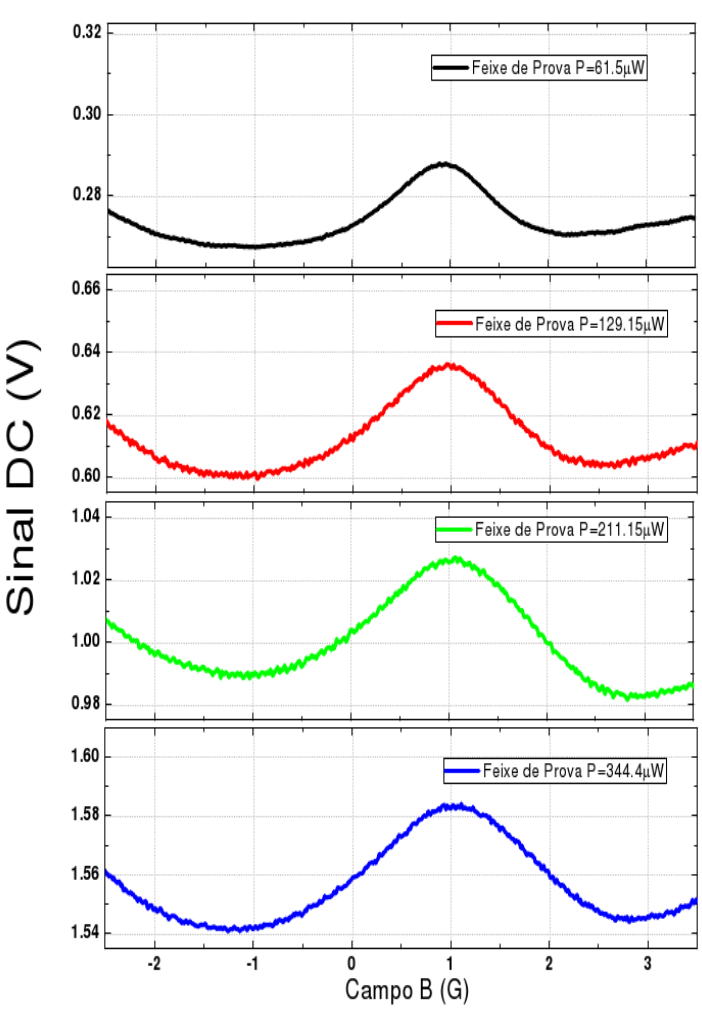

(a)

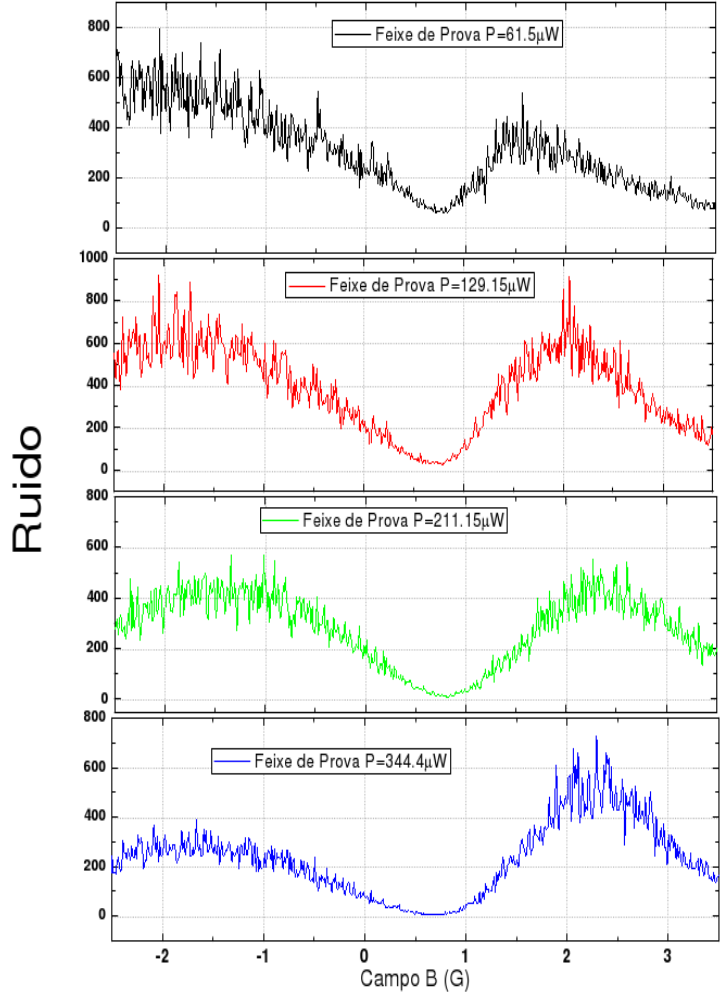

(b)

Figura 4.20: (a) Espectro de absorção do sinal DC em condição de Hanle-EIT, para as potências $P=$ $61,129,211,344 \mu \mathrm{W}$ do laser. (b) Espectro de ruídos para os respectivos espectros de absorção do sinal DC.

para não criar muitas flutações do campo magnético no centro da câmara onde se localiza a nuvem atômica, fizemos varreduras do campo num intervalo de \pm 3 Gauss. O que é um valor suficiente para fazer uma espectroscopia de absorção resolvida.

Para fazer o espectro de absorção, de ruido e de correlação, empregamos a mesma montagem experimental da seção anterior, porém, usamos só um feixe com polarização linear, e após ele interagir com os átomos, separamos as componentes $\sigma_{+} \mathrm{e}$ $\sigma_{-}$com as lâminas de $\lambda / 4$. A figura 4.19.a mostra a montagem experimental para 
a experiência de Hanle-EIT com luz linearmente polarizada.

A figura 4.20.a mostra o espectro de absorção de uma componente $\sigma_{ \pm}$, para as potências $P=61,129,211,344 \mu \mathrm{W}$. A figura 4.20.b mostra o espectro de ruído que corresponde às potências do sinal DC. Da mesma forma que no caso da seção anterior, o sinal DC apresenta um alargamento por potência. Esses espectros foram obtidos mantendo o laser em ressonância. No entanto, quisemos estudar o que acontecia quando deslocávamos a frequência do laser.

A figura 4.21 mostra os espectros de absorção para valores de dessintonia do laser $\delta=0 \mathrm{MHz}, 1 \mathrm{MHz}$ e $2 \mathrm{MHz}$. Podemos observar que a simetria do perfil se quebra, perdendo resolução. Isto acontece porque a dessintonia muda a frequência para a qual os campos entram en condição de EIT, porém, essa condição não acontece numa ressonância atômica. Isto é equivalente à presença de campos magnéticos no caso da seção anterior.

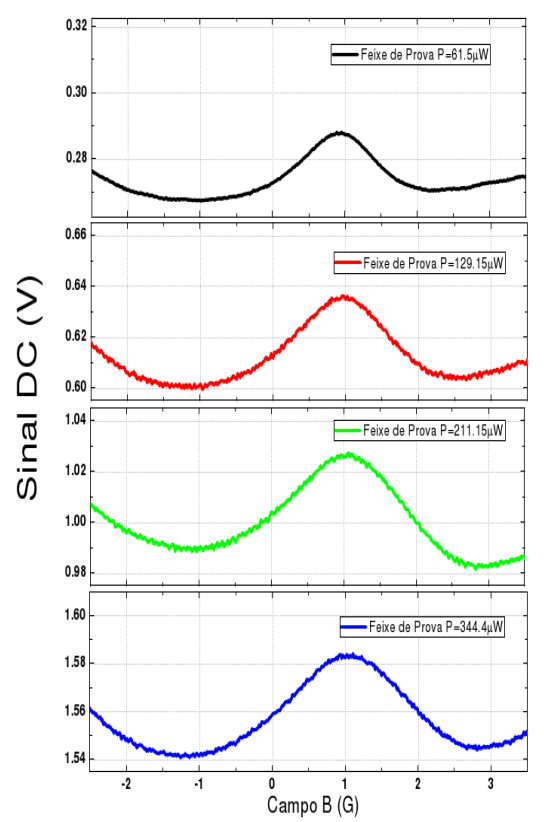

(a)

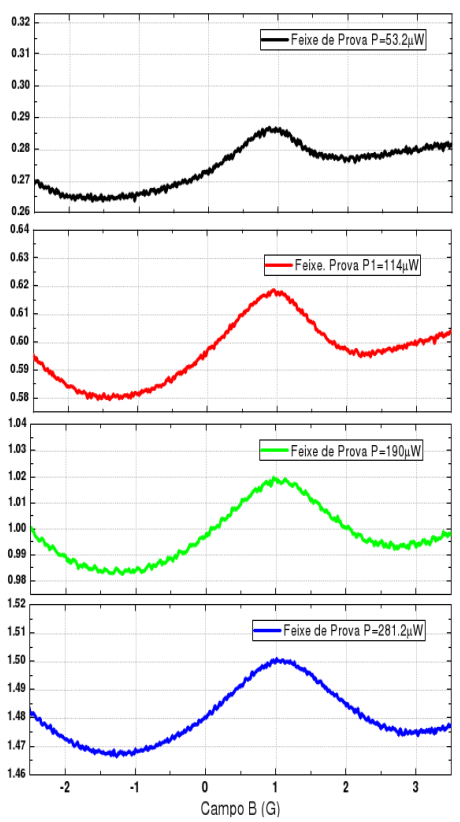

(b)

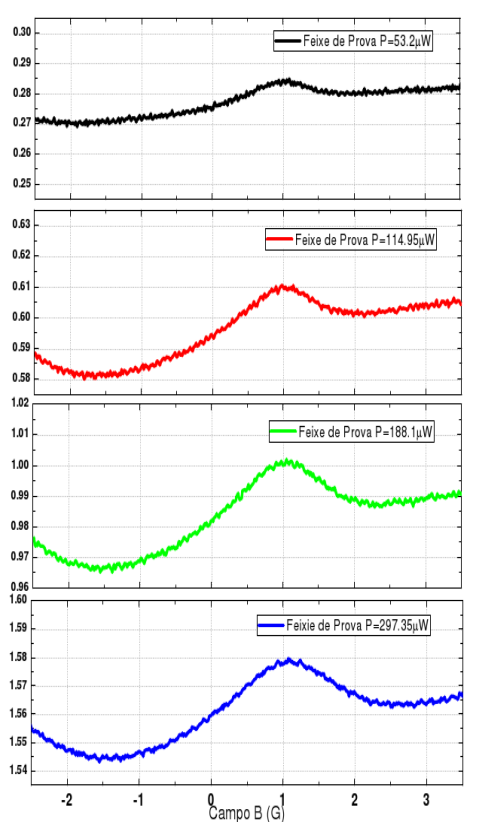

(c)

Figura 4.21: Espectro de absorção do sinal DC em condição de Hanle-EIT para (a) Dessintonia $\delta=0 \mathrm{MHz}$, (b) Dessintonia $\delta=1 \mathrm{MHz}$ e (c) Dessintonia $\delta=2 \mathrm{MHz}$.

Juntamente com os espectros de absorção do sinal DC e do ruído, da figura 4.20, obtemos os espectros de correlação para os diferentes valores de dessintonia, como mostra a figura 4.22. Da mesma forma que no sistema da seção anterior, temos a transição de correlação para anti-correlação comforme aumentamos a intensidade dos feixes. No entanto, podemos observar uma assimetria do fator C (correlação) ao redor da ressonância, o que não acontecia no caso anterior. Isto pode ter origem 
no efeito Faraday, em que a polarização linear do campo eletromagnético muda na presença de campos magnéticos. Então, quando o laser atravessa a região atômica, o campo magnético aplicado pode criar uma pequena variação na polarização linear, de modo que uma das componentes $\sigma_{+}$ou $\sigma_{-}$, seria beneficiada. Isto aumentaria a absorção de um campo e diminuiria a absorção do outro. Esse desbalanceio de absorção induziria a uma anti-correlação entre os dois campos. Outra possibilidade, é a presença de algum campo espúreo que surge na modulação propria do campo de compensação, que gera uma dessintonia, que não é compensada eficientemente.

Embora tenhamos o efeito de assimetria no perfil de correlação, podemos analisar o que acontece com $C$ à medida que aumentamos a dessintonia do laser. Os campos ficam correlacionados conforme aumentamos a dessintonia do laser, até perder qualquer tipo de anti-correlação (o que acontece $\operatorname{com} \delta=2 \mathrm{MHz}$ ).

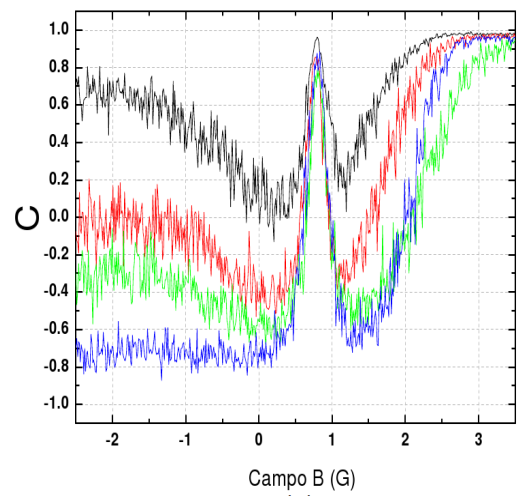

(a)

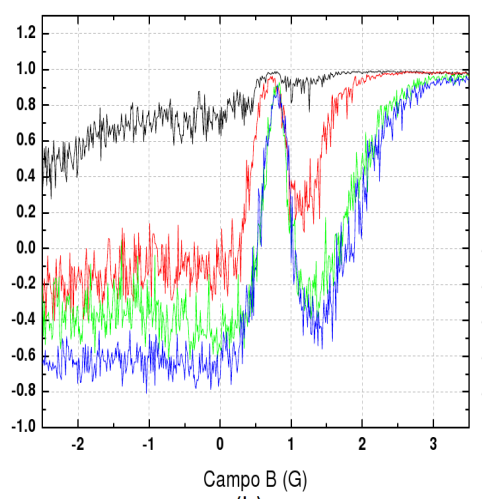

(b)

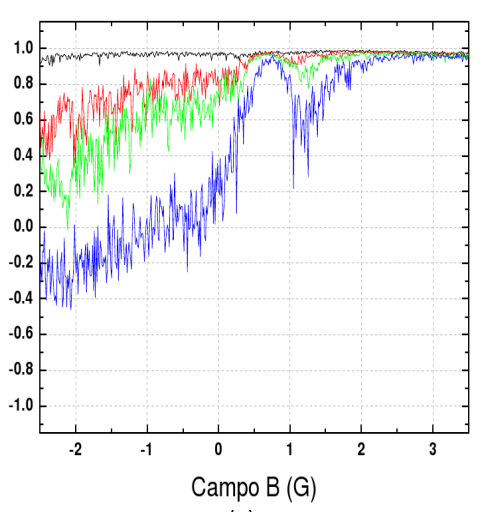

(c)

Figura 4.22: Espectro de correlação em condição de Hanle-EIT para (a) Dessintonia $\delta=0 \mathrm{MHz},(\mathbf{b})$ Dessintonia $\delta=1 \mathrm{MHz}$ e (c) Dessintonia $\delta=2 \mathrm{MHz}$.

Apesar da assimetria acontecer, o pico central aparentemente é livre dessa assimetria para algumas dessintonias, de modo que fazendo um ajuste Loreztiano do pico de correlação, unicamente para dessintonias de $\delta=0$ e $1 \mathrm{MHz}$ (porque no caso de $\delta=2 \mathrm{MHz}$, a forma do pico é afetada, o que não permie fazer o ajuste), na figura 4.23 , comparamos a largura a meia altura do pico de correlação com a largura do sinal DC, em função da intensidade dos feixes.

Para tentar entender melhor por que obtemos esse efeito de assimetria no espectro de correlação, fizemos uma mudança de base na polarização dos campos. Antes do laser interagir com os átomos, separamos as componentes verticais das horizontais de polarização, de modo que quando passam pela lâmina $\lambda / 4$, obtemos novavemente dois feixes com polarizações circulares opostas $\sigma_{+}$e $\sigma_{-}$, como mostra a figura 4.19.b. Para esta situação obtemos a espectroscopia de correlação da figura 4.24.a.

Esta espectroscopia poderia confirmar a hipótese de que, no caso da polarização 

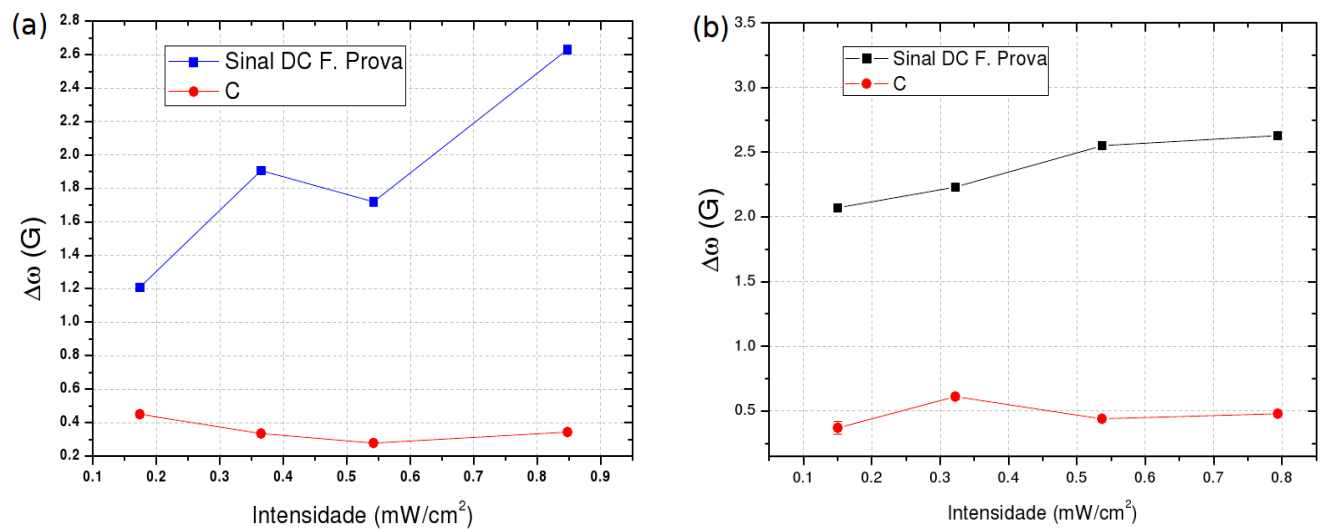

Figura 4.23: Largura do pico de Hanle-EIT do sinal DC e da correlação, em função da intensidade do laser. (a) para dessintonia $\delta=0 \mathrm{MHz}$ e (b) para dessintonia $\delta=1 \mathrm{MHz}$.
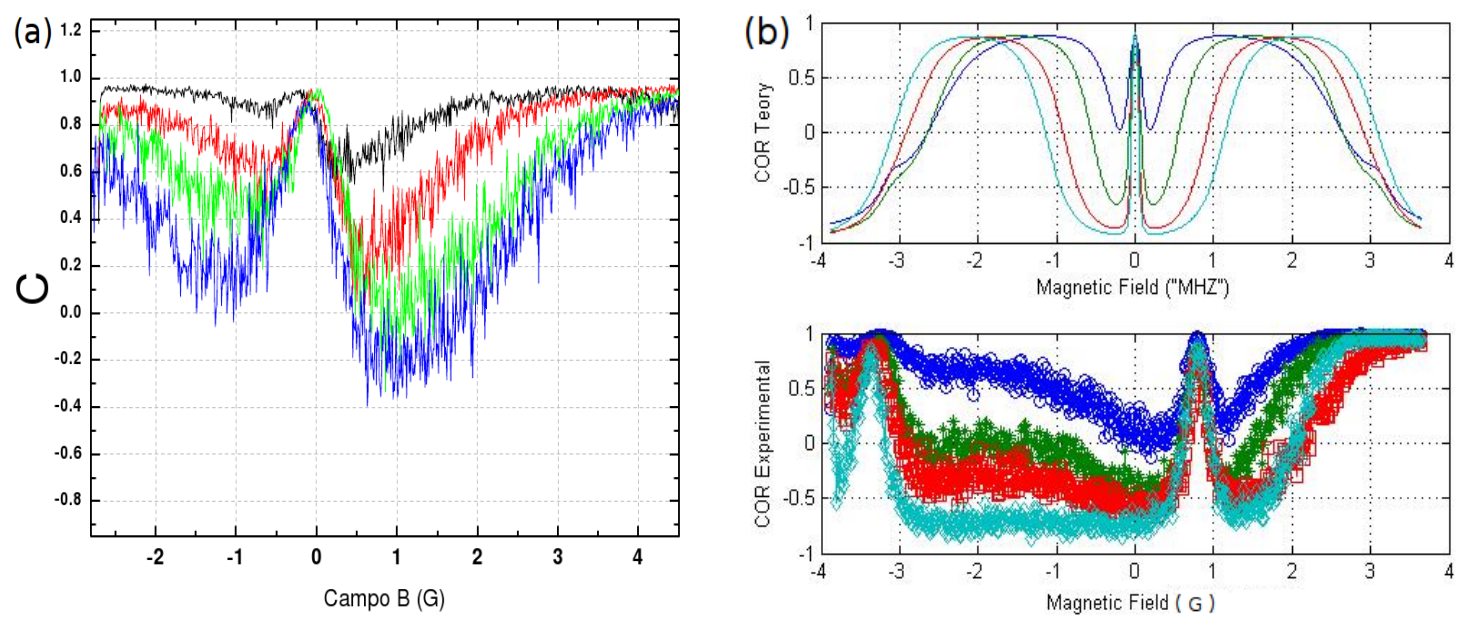

Figura 4.24: (a) Espectro de correlação com luz polarizada na base $\sigma_{ \pm}$. (b) Comparação do espectro de correlação teórico e esperimental em condição Hanle-EIT

linear, a assimetria dos espectros está influenciada por alguma mudança na polarização linear durante a interação com os átomos, ou por qualquer tipo de mecanismo magnético que altere os níveis atômicos, beneficiando uma das componentes. No entando, devemos considerar que este sistema é um sistema degenerado, em que estamos deslocando todos os níveis Zeeman ao mesmo tempo, dificultando assim, o entendimento da dinâmica complexa da população atômica em cada um dos níveis.

Em colaboração com o Prof. Luciano Cruz da UFABC, usamos o modelo de difusão de fase do capítulo 2, levando em conta a presença dos campos magnéticos na dinâmica dos níveis atômicos para descrever o ruído e a correlação dos campos de luz, em condição de Hanle-EIT, da seção 1.5. Calculando de forma númerica a correlação 
dos campos interagindo com o meio atômico, considerando a degenerescência dos níveis hiperfinos, obtemos o espectro de correlação da figura 4.24.b. Este espectro reproduz a transição de correlação para anti-correlação conforme aumentamos a intensidade dos feixes. No entando, este não mostra a assimetria presente no espectro experimental. Porém, a simetria do espectro teórico esta em concordância com o espectro obtido em 4.24.a, feito numa base de polarização diferente.

Uma vantagem desta espectroscopia em condição de Hanle-EIT, é a manipulação da dessintonia do laser mediante o controle dos níveis atômicos, aplicando campos magnéticos, sem precisar manipular diretamente a frequência do laser através de moduladores acusto-oticos, como foi feito na seção anterior. Estes podem introduzir um excesso de ruído na espectroscopia de ruído e correlação. Aplicando o campo magnético para o controle dos níveis, não observamos exceso de ruído. Em contrapartirda, a degenerescência dos níveis hiperfinos, podem ter contribuições mais complexas nos espectros de Hanle-EIT, do que no caso simples de EIT. O modelo teórico é mais complexo, porque devemos considerar todas as possibilidades de acoplar os campos de luz com vários sistemas $\Lambda$ de EIT.

Daí que podemos pensar em trabalhar com átomos de Rubídio 87, de modo que podemos usar a transição $F=1$ para $F=0$, de modo que a estrutura de níveis Zeeman é mais simples, diminuido a degenerescência dos níveis. Nesse caso, a manipulação dos níveis com campos magnéticos, pode ser muito mais proveitosa. 


\section{CAPÍTULO 5}

\section{Conclusões e perspetivas}

Este trabalho é a continuação de uma linha de pesquisa que vem sendo desenvolvida no LMCAL (Laboratorio de Manipulação Coerente de Átomos e Luz), na construção, preparação e caracterização da Armadilha Magneto-Ótica (MOT), em que resfriamos e aprisionamos átomos frios de Rubídio. De 2008 a 2009 foi construída a MOT, cuja etapa inicial precisou da caracterização do número de átomos e da temperatura da nuvem. Posteriormente, foi feita uma espectroscopia da nuvem, em que foi necessário desenvolver programas de controle da nuvem, para sincronizar o carregamento com o feixe para espectroscopia. Após este processo, estudamos fenômenos coerentes como Transparência Electromagneticamente Induzida (EIT). O trabalho também deu continuidade a alguns trabalhos feitos em 2005 e 2009, que estudávam as flutuações dos campos eletromagnéticos interagindo com átomos de Rubídio quentes em células de vapor, em condição de EIT.

Durante a evolução do mestrado, estudamos quais os tempos dentro dos quais é possível fazer as medidas de EIT; quais os níveis atômicos necessários para obter o espectro de EIT mantendo a frequência dos laser sintonizada, e quão susceptível é o nosso sistema na presença de campos magnéticos.

Adaptamos o sistema de deteção de ruído desenvolvido no laboratório, em experimentos como o Oscilador Paramétrico Ótico. Conseguimos demodular as flutuações de intensidade dos campos eletromagnéticos no experimento com os átomos frios. $\mathrm{Na}$ deteção das flutuações do campo de luz, foi empregando um sistema de aquisição de dados digitais controlados por computador. Este esquema computarizado permite acoplar e sincronizar o sistema de controle dos átomos na MOT com o sistema de deteção. 
Considerando todo o processo de preparação do experimento, estudamos de forma experimental e teórica, os campos eletromagnéticos e suas flutuações, interagindo com átomos frios de Rubídio, com dois tipos de fenômeno presentes no sistema: EIT e Hanle-EIT. Especificamente, fizemos espectroscopias de absorção, de ruído e de correlação em condição de EIT e Hanle-EIT. Analisando os espectros de absorção e correlação, achamos um alargamento por potência do sinal DC, enquanto a correlação, é livre de alargamento por potência. Isto permite pensar que a caracterização do tempo de coerência característico na condiação de EIT (relacionado com a largura do pico ao redor da condição Raman), pode ser caracterizado pela correlação, porque filtramos qualquer efeito devido à potência da luz.

Inicialmente estudamos o fenômeno de EIT, em que manipulamos de forma independente a frequência dos lasers, modelando o sistema mediante o modelo de Difusão de Fase para um sistema de três níveis. Os espectros obtidos de absorção, ruído e correlação, estão em concordância com os espectros experimentais. Isto mostra que mediante um modelo simples de 3 níveis, conseguimos descrever as características do fenômeno de EIT, como o não alargamento do pico de correlação e a transição de correlação e anti-correlação, de sistemas mais complexos que posuem degenerescência nos níveis, que fazem a interação átomo-luz mais complexa.

Por outro lado, dedicamos uma parte deste trabalho para estudar o fenômeno de Hanle-EIT e as suas características nos espectros de ruído e correlação. Notamos que é possível observar os mesmos efeitos do caso de EIT, porém com algumas dificuldades no controle do campo magnético. No caso em que trabalhamos com luz linearemente polarizada, observamos um perfil de absorção tipo-EIT em que a correlação apresenta uma assimetria em relação ao caso de EIT. Mostramos que mudando a base de polarização, separando as componentes de polarização $\sigma_{+}$e $\sigma_{-}$, recuperamos a simetria do perfil de correlação. O modelo teórico está em concordância com os espectros para campos com polarização na base $\sigma_{+}$e $\sigma_{-}$.

A ideia de implementar o sistema de deteção usando um demodulador para medir as flutuações da luz numa frequência de análise dada, em vez de usar diretamente um Analisador de Espectro, está relacionado às perspectivas deste trabalho. Este esquema de deteção já é empregado no laboratório, na experiência de emaranhamento tripartite em variáveis contínuas usando um Oscilado Paramétrico Ótico (OPO), o que faria mais fácil o acoplamento dos dois experimentos. A partir do OPO, podemos gerar campos coerentes emaranhados, de modo que, se um deles for acoplado aos átomos frios, podemos emaranhar os estados atômicos ao feixe sinal e complementar, criados no OPO. Isto envolve o esquema digital de medidas de ruídos e correlação, para o qual o sistema de átomos já estaria adaptado. No entanto, para essa integração, precisamos construir um OPO que opere na frequência de interação dos átomos de Rubídio. 
Outro projeto que esta sendo desenvolvido em paralelo a este trabalho, é a construção de um laser de Ti:Safira, com o qual podemos criar campos coerentes. Usando este laser podemos bombear um OPO operando na frequência do Rubídio, e também podemos fazer mistura de quatro ondas (4WM), com a qual é possível atingir um nível de compressão de ruído (mais de 6dB) abaixo do Shot-noise [16, 17]. Isto envolve o fenômeno de EIT e todas as características estudadas neste trabalho.

A integração dos átomos frios na MOT com outros experimentos em curso no laboratório depende fortemente da medida de ruído dos campos eletromagnéticos. Para medir as flutuações da luz, devemos isolar o sistema de fontes externas que possam criar excesso de ruído, como os moduladores acusto-óticos. Podemos explorar o efeito de Hanle-EIT como um mecanismo com o qual podemos obter uma espectroscopia dos átomos frios, sem manipular diretamente a frequência do laser com AOMs, evitando assim, introduzir qualquer tipo de exceso de ruído. No entanto, a degenerescência dos níveis pode dificultar o estudo teórico e experimental, do efeito de Hanle-EIT. É por isso que resfriaremos átomos de Rubídio 87, em níveis que vão permitir sintonisar o laser de prova e de controle, na transição $F=1$ a $F=0$, obtendo assim um sistema de níveis com mínima degenerescência para configurações $\Lambda$ em condição de EIT. 


\section{APÊNDICE A}

\section{Software de controle e aquisição}

Durante a construção da MOT de Rubídio no LMCAL, foi necessário desenvolver um programa de controle da nuvem, com o qual se consiguiu fazer a sua espectroscopia. Dado que neste trabalho queríamos fazer espectroscopia de ruído, também precisamos usar um programa de aquisição de dados, já desenvolvido no laboratório, a exceção de algumas modificações. Porém, mesmo sendo ferramentas tão importantes para o experimento, estes programas não tinham sido descritos anteriormente. Por esta razão, dedicamos um Apêndice com o intuito de dar uma orientação para o usuario dos programas durante o experimento.

Incialmente, descreveremos o programa que controla a nuvem átomica na MOT, e posteriormente mostraremos como funciona o programa de aquisição de dados. Um ponto importante a entender desse programas, é o acoplamento dos dois programas que vão sincronisar as medidas de espectroscopia.

\section{A.1 Programa de Controle da MOT}

O painel do programa de controle feito em LabView, é mostrado na figura A.1. Dentro do painel, na aba Measurement Selection escolhemos o tipo de medida a ser feita. No caso de interesse, escolhemos Probe Scanning on MOT. Na parte direita da aba, aparece o tempo de carrega da nuvem descrito por Time out, no nosso caso de $800 \mathrm{~ms}$, tempo suficiente para cargar a nuvem atômica. Depois deste tempo de carga, no tempo $t=0$ começa a sequência de pulsos de controle do dispositvos de tensão que controla os laser de resfriamento, o campo magnético de aprisionamento, o feixe de prova para espectroscopia e o feixe de rebombeio. $\mathrm{Na}$ 


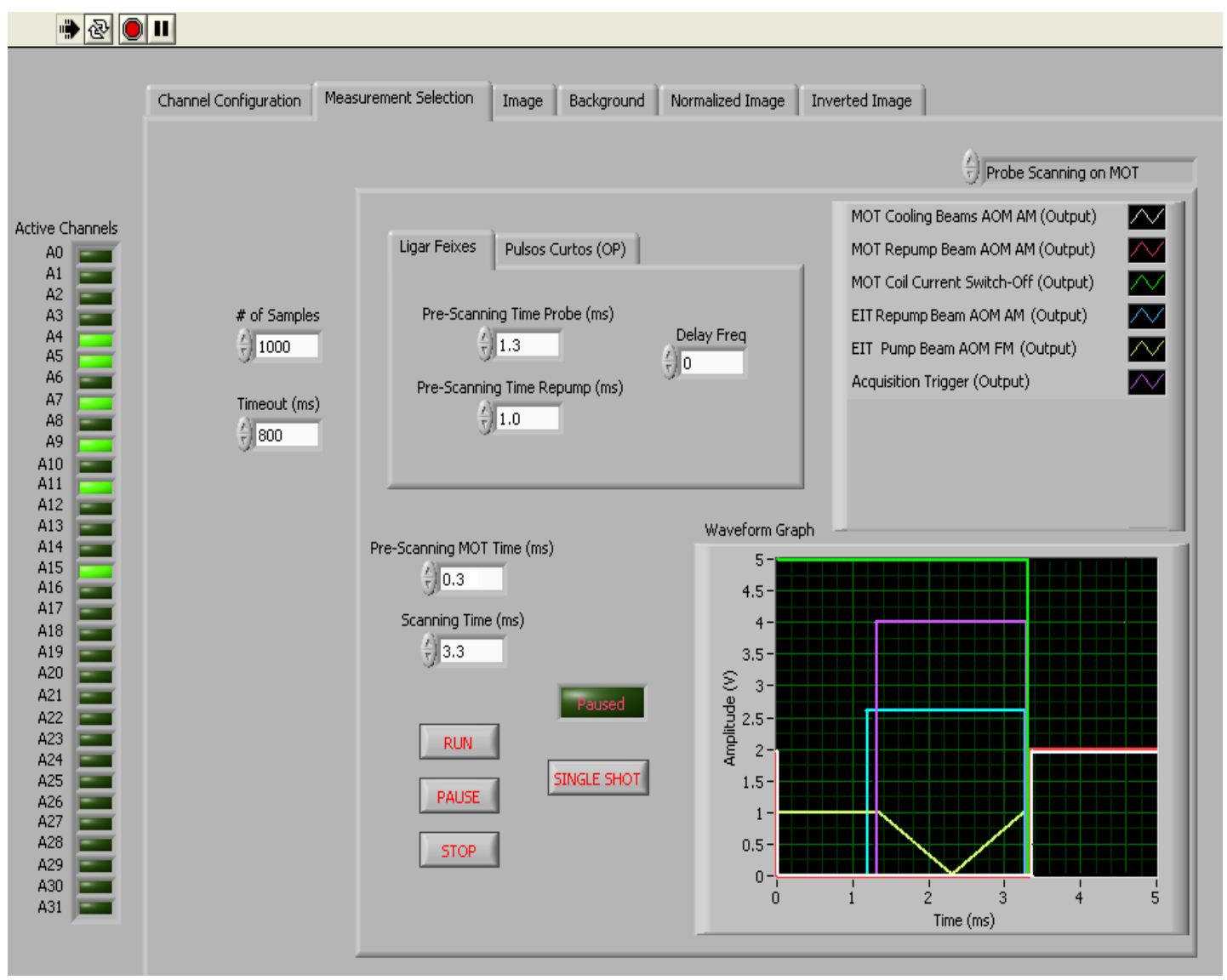

Figura A.1: Aba Measuremente Selection, do painel do programa de controle da MOT.

aba Ligar Feixes, podemos controlar o tempo em que é ligado o feixe de prova para fazer a espectroscopia de EIT a partir do tempo $t=0$, usando a entrada Pre-Scanning Probe, que no caso é de 1.3ms. A entrada Pre-Scanning Time Repump controla o tempo em que é ligado o campo de rebombeio, para o controle de população atômica. Durante a experiência trabalhamos com um tempo de $1 \mathrm{~ms}$ de espera para ligar o rebombeio.

$\mathrm{Na}$ parte inferior do panel, mostramos o controle dos feixes de resfriamento e o tempo de varredura de tensão, no qual é feita a espectroscopia. Na entrada PreScanning MOT Time, controlamos o tempo em que desligamos estes feixes. Nas experiências trabalhamos com $0,3 \mathrm{~ms}$, mas às vezes usávamos $0,6 \mathrm{~ms}$, para tentar manter a nuvem de átomos o mais fria possível antes de fazer a espectroscopia. Por outro lado, na entrada Scanning, controlamos o tempo que durava o processo todo. Com isso, o programa calcula o tempo de varredura de frequência para fazer a espectroscopia. No nosso caso, o programa calcula $2 \mathrm{~ms}$ para aumentar e diminuir 
a tensão de entrada FM do dispositivo (linha amarela) que controla a frequência do AOM.

O programa desliga automaticamente o campo magnético de aprisionamento e o rebombeio da MOT, portanto, não tem uma entrada de tempo, como os outros canais.

Na parte superior direita, o painel mostra os canais de saída que estão ativados. São eles:

- MOT cooling beams AOM AM: Mostra que o canal dos feixes de resfriamento esta ativado. Este canal controla a entrada de modulação de amplitude (AM) do dispositivo eletrônico (VCO) conectado ao AOM.

- MOT Repump Beam AOM AM: Este canal controla a entrada de modulação de amplitude (AM) do dispositivo eletrônico (VCO) conectado ao AOM para o feixe de Rebombeio da MOT.

- MOT Coil Current Switch-Off: Mostra que o canal que controla o desligamento do campo magnético de aprisionamento da MOT, está ativado.

- EIT Repump Beam AOM AM: Este canal controla a entrada de modulação de amplitude (AM) do VCO conectado ao AOM para o feixe de Rebombeio, que controla a população atômica para a espectroscopia do feixe de prova.

- EIT Pump Beam AOM FM: Mostra que o canal que controla a modulação de frequência (FM) do dispositivo eletrônico conectado ao AOM esta ativado. Este canal posui uma variação de tensão linear, que controla a frequência do feixe de prova, de forma linear.

- Acquisition Trigger: Este canal cria um pulso de Trigger, para ativar o processo de aquisição de dados com o programa de aquisição (este programa será descrito na próxima seção), durante a espectroscopia. Se este canal não for ativado, não é possível fazer espectroscopia de ruído e correlação.

Se tivermos mais canais ativados, apareceriam nessa aba. Cada um dos canais está acompanhado com uma linha de cor especifica, que corresponde à linha no diagrama de pulsos mostrado na parte inferior direita do panel, reproduzendo a sequência de pulsos da figura 4.3 .

Finalmente para iniciar o programa de controle, apertamos o botão Run. Devemos conferir, se os dispositivos VCO que controlam todos os AOMs estão desligados, de maneira que os pulsos enviados pelo programa para estes dispositivos controlem 
a tensão no intervalo de tempo que dura a medição. Para suspender ou parar definitivamente o programa, apertamos os botões Pause ou Stop, respectivamente.

Para ativar e desativar um canal de controle, devemos mudar para a aba Channel Configuration. Nela observamos várias colunas que caracterizam cada canal. Se o canal estiver ativado, o botão ascende em cor verde claro, e se estiver desativado, o canal desliga e mostra uma cor verde escuro, como mostra a figura A.2. As colunas, descrevem várias propiedades de cada canal. A primeira corresponde

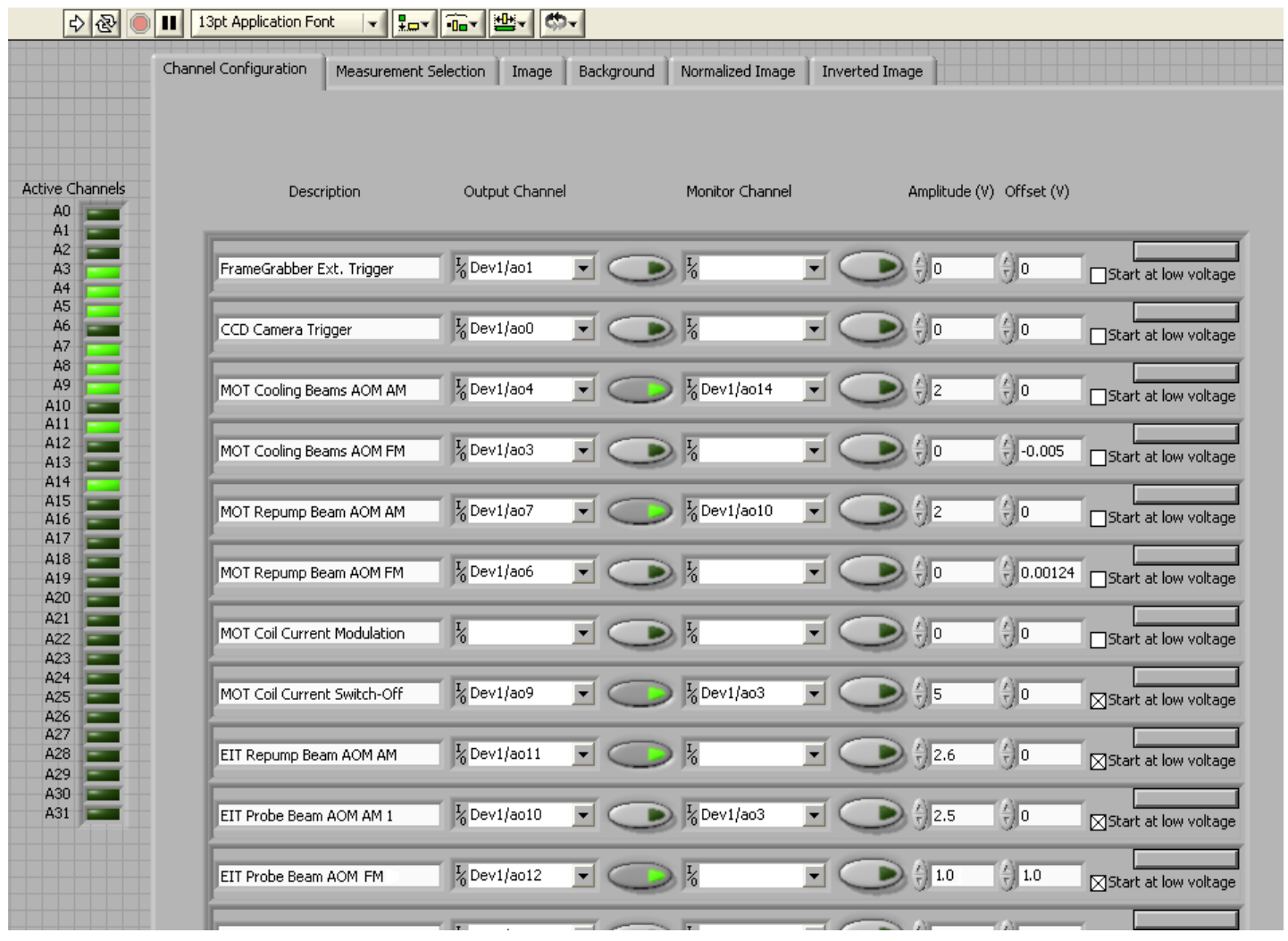

Figura A.2: Aba Channel Configuration, em que ativamos os canais a serem usados, do programa de controle da MOT.

ao nome do canal. A segunda coluna, descreve a porta de saída no qual podemos conetar o dispositivo que queremos controlar. A terceira coluna, permite ativar ou desativar uma cópia do sinal de saída, para ter um controle da saída do sinal gerado pelo programa. A quarta e quinta coluna, mostram a Amplitude e o Offset da tensão $(\mathrm{V})$, respectivamente, aplicada num canal dado. 


\section{A.2 Programa de Aquisição}

Este programa é um pouco mais complexo do que o anterior, de modo que vamos dividir em duas partes. A primeira aparece na figura A.3, dividida em três janelas. A primeira delas, Acquisition, corresponde ao sistema de aquisição do programa. Com a entrada \# of Samples, determinamos quantos dados vamos adquirir, numa taxa de de aquisição dada, indicada em Nominal Sample Rate. No caso da experiência,

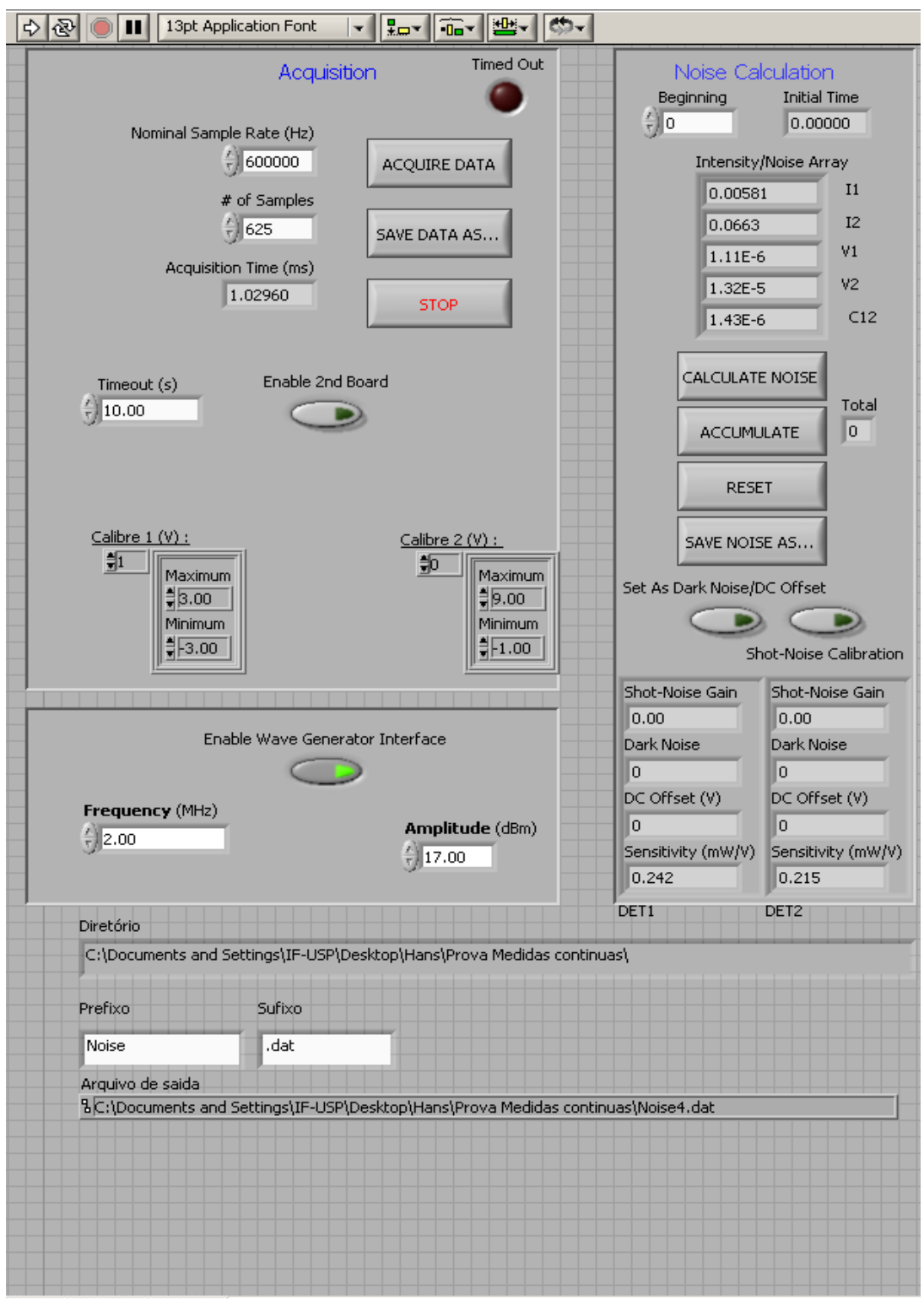

Figura A.3: Primeira parte do panel do programa de aquisição. 
usamos uma taxa de $600 \mathrm{kHz}$, limitado pela taxa do demodulador. Com esses dois valores, o programa calcula durante quanto tempo ele adquiri dados, mostrando o tempo calculado em Acquisition Time. Para começar a adquisição de dados, apertamos o botão Acquire Data. O inicio da adquisição de dados é sincronisado com o Trigger gerado pelo programa de controle da MOT, descrito anteriormente, durante o qual vamos fazer a espectroscopia de ruído. Se o programa de controle da MOT não enviar o sinal de ativação num tempo de 10 s, o programa ascende o botão Time out. Este é um bom indicador da sincronisação dos dois programas.

Uma vez que os dados são adquiridos, podemos salvá-los apertando o botão Save data as.... O programa salvará os dados na direção do diretório fornecido pelo usuario. O nome e o tipo de arquivo (.dat no nosso caso), são especificados pelo usuario na parte inferior do painel em Prefixo e Sufixo, de modo que quando apertar o botão para salvar, o programa salve automaticamente os dados com esse nome, nesse diretório.

Na janela abaixo da janela Acquisition, temos o controle da frequência e amplitude do gerador de funções conetada ao demodulador, que determina a frequência na qual demodulamos o sinal de alta frequência (HF) que fornece o detetor. Para ativar ou desativar esta interface, apertamos o botão Enable Wave Generator Interface. No experimento usamos uma frequência de análise de $2 \mathrm{MHz}$, e um aplitude de $17 \mathrm{dBm}$, na entrada do demodulador.

A placa de aquisição, possui quatro canais de entrada, dos quais, dois correspondem ao sinal DC de cada um dos detetores, e os dois restantes às flutuações demoduladas de cada um dos detetores. Na terceira janela da primera parte do programa, chamada Noise Calculation, temos os indicadores da média do sinal DC e do quadrado do sinal HF de cada um dos detetores. Também indica a média do produto dos sinais HF dos detetores. Estas médias são feitas no tempo de aquisição.

Quando fazemos uma aquisição de dados, podemos aproveitar a função Accumulate, que faz o programa calcular o valor médio do sinal DC e o ruído, contando o número da aquisição feita. Após acumular, podemos salvar os dados brutos, fazer uma aquisição nova de dados, de modo que se acumulamos, ao salvar de novo, o programa salva introduzindo no nome do arquivo, o número que corresponde a essa aquisição. Isto permite salvar várias aquisições consecutivas, de forma eficiente, sem perder muito tempo mudando o nome do arquivo manualmente.

Na figura A.4 mostramos a segunda parte do programa, que mostra a visualização dos dados adquiridos em cada um dos canais, em função do tempo. Na aba Channel0 e Channel1, mostramos o sinal HF o sinal DC, respectivamente, para cada um dos detetores. Na parte inferior do programa, mostramos 6 abas, cada uma, com informação do ruído calculado quando usamos a função Accumulate, descrita 
anteriormente. Estas abas são organizadas da seguinte forma:Page1 e Page2 correponde ao ruído de cada um dos detetores em função do sinal DC. ; Page3 mostra a correlação de ruído em função do sinal DC. Page4 Gráfica o ruído do canal $2 \mathrm{em}$ função do ruído do canal 1;Page5 e Page6. Corresponde à diferença e à soma dos ruído, em função do sinal DC.

Este conjunto de 6 abas é muito útil na hora de fazer a calibração de ShotNoise, porque permite visualizar quão linear é a diferença dos ruídos em função da intensidade dos feixes.

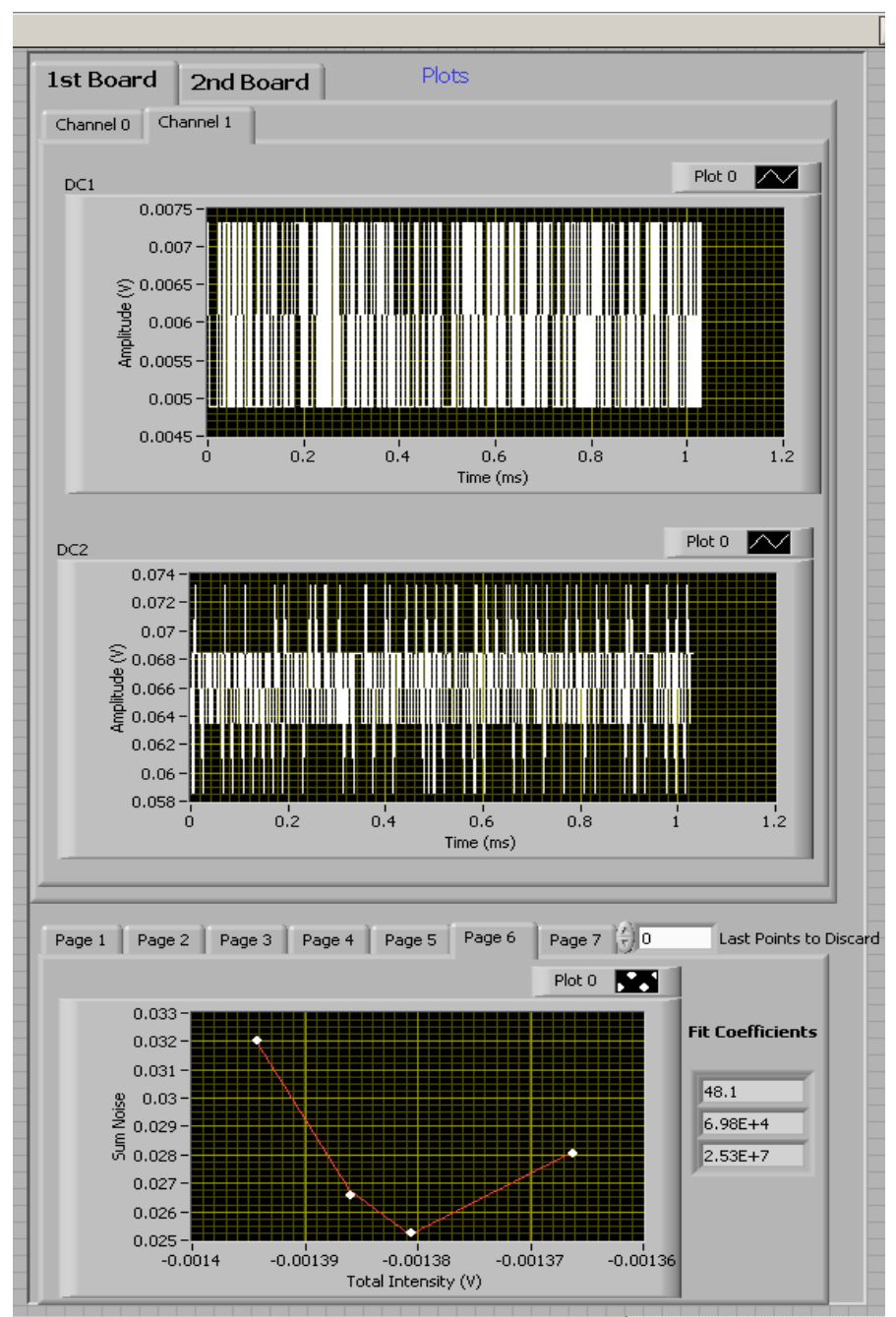

Figura A.4: Segunda parte do panel do programa de aquisição. 


\section{Bibliografia}

[1] B. Podolsky A. Einstein and N. Rosen. Can quantum-mechanical description of physical reality be considered complete? Phys. Rev., 47:777-780, 1935.

[2] P. Grangier A. Aspect and G. Roger. Experimental realization of einsteinpodolsky-rosen-bohm gedankenexperiment: A new violation of bell's inequalities. Phys. Rev. Lett., 49:91-94, 1982.

[3] Isaac L. Chuang. Michael A. Nielsen. Quantum Computation and Quantum Information. Springer-Verlag, 1st edition, 2000.

[4] Alan Migdall et al. Three-dimensional viscous confinement and cooling of atoms by resonance radiation pressure. Phys. Rev. Lett., 55:48-51, 1985.

[5] J. E. Bjorkholm Alex Cable Steven Chu, L. Hollberg and A. Ashkin. First observation magnetically trapped neutral atoms. Phys. Rev. Lett., 54(24), 1985.

[6] A. Heidmann C. Salomon A. Aspect, J. Dalibard and C. Cohen-Tannoudji. Cooling atoms with stimulated emission. Phys. Rev. Lett., 57:1688-1691, 1986.

[7] Claude N. Cohen tanoudji and D.W. Phillips. New mechanisms for laser cooling. Phys. Today, October:33-40, 1990.

[8] J. E. Bjorkholm et al. Observation of focusing of neutral atoms by the dipole forces of resonance-radiation pressure. Phys. Rev. Lett., 41:1361, 1978.

[9] M.R. Matthews C.E. Wieman M.H. Anderson, J.R. Ensher and E.A. Cornell. Observations of bose-einstein condensation in a dilute atomic vapor. Sci, 269:198-201, 1995. 
[10] Stephen E. Harris. Electromagnetically induced transparency. Phys. Today, July:36-42, 1997.

[11] A. Imamoglu K.J. Boller and S.E. Harris. Observation of electromagnetically induced transparency. Phys. Rev. Lett., 66:2593-2596, 1991.

[12] S.A. Hopkins; E. Usadi; H.X. Chen; A.V. Durrant. Electromagnetically induced transparency of laser-cooled rubidium atoms in three-level $\lambda$-type systems. $O p t$. Comm., 138:185-192, 1997.

[13] Tony van der Veldt; Jean-Fraqois Roth; Philippe Grelu; Philippe Grangier. Nonlinear absorption and dispersion of cold rb 87 atoms. Opt. Comm., 137:420426, 1997.

[14] D.F Walls and G.J. Milburn. Quantum Optics. Springer, 2nd edition, 2008.

[15] Min Xiao Ling-An Wu and H. J. Kimble. Squeezed states of light from an optical parametric oscillator observation of electromagnetically induced transparency. J. Opt Soc. Am. B, 4(10), 1987.

[16] ; A. M. Marino; V. Boyer; K. M. Jones; R. C. Pooser and P. D. Lett. Lownoise amplification of a continuous-variable quantum state squeezed states of light from an optical parametric oscillator observation of electromagnetically induced transparency. Phys. Rev. Lett., 103(010501), 2009.

[17] R. C. Pooser; A. M. Marino; V. Boyer; K. M. Jones; and P. D. Lett. Quantum correlated light beams from non- degenerate four-wave mixing in an atomic vapor: the $\mathrm{d} 1$ and $\mathrm{d} 2$ lines of ${ }^{85} \mathrm{rb}$ and ${ }^{87} \mathrm{rb}$. Opt. Express, 17(19), 2009.

[18] Lu-Ming Duan. Inseparability criterion for continuous variable systems. Phys. Rev. Lett., 84(2722), 2000.

[19] 1; A. M. Marino; V. Boyer;R. C. Pooser and P. D. Lett. Entangling light in its spatial degrees of freedom with four-wave mixing in an atomic vapor. Chem. Phys. Chme, 10(755-760), 2009.

[20] V. Boyer; C. F. McCormick; E. Arimondo and P. D. Lett. Ultraslow propagation of matched pulses by four-wave mixing in an atomic vapor. Phys. Rev. Lett., 99(143601), 2007.

[21] M. Fleischhauer and M.D. Lukin. Quantum memory for photons: Dark-state polaritons. Phys. Rev. A., 65(022314), 2002.

[22] Danielle A. Braje; Vlatko Balic; Sunil Goda; G. Y. Yin and S. E. Harris. Frequency mixing using electromagnetically induced transparency in cold atoms. Phys. Rev. Lett., 93(18), 2004. 
[23] D. F. Phillips; A. Fleischhauer; A. Mair; R. L. Walsworth and M. D. Lukin. Storage of light in atomic vapor. Phys. Rev. Lett., 86(05), 2001.

[24] Brian Julsgaard et al. Experimental demonstration of quantum memory for light. Nature, 432(25), 2004.

[25] T. Chaneliere1 et al. Storage and retrieval of single photons transmitted between remote quantum memories. Nature, 438(04315), 2005.

[26] Kazuhito Honda et al. Storage and retrieval of a squeezed vacuum. Phys. Rev. Lett., 100(093601), 2008.

[27] D. Mooretti et al. Collapses and revivals of stored orbital angular momentum of light in a cold-atom ensemble. Phys. Rev. A., 79(023825), 2009.

[28] M.Martinelli et al. Noise spectroscopy of non-linear magneto optical resonances in rb vapor. Phys. Rev. A., 69(043809), 2008.

[29] Luciano Cruz. Flutuaçõess de campo electromagnéticos interagindo com meios atômicos coerentemente preparados. PhD thesis, Universidade de São Paulo, 2004 .

[30] Felippe Barbosa. Flutuaçõess de campo electromagnéticos no regime de transparência eletromagnéticamente induzida, Dezembro 2009.

[31] Vladimir A. Sautenkov et al. Switching between photon-photon correlations and raman anticorrelations in a coherently prepared rb vapor. Phys. Rev. A., 72(065801), 2005.

[32] Tigran S. Varzhapetyan et al. Intensity correlations in a coherently prepared rb vapor in a magnetic field. Opt. Comm., 282:39-43, 2009.

[33] L.S. Cruz et al. Laser-noise-induced correlations and anti-correlations in electromagnetically induced transparency. Eur. Phys. J. D., 41:531-539, 2006.

[34] Yanhong Xiao et al. Electromagnetically induced transparency with noisy lasers. Phys. Rev. A., 80(041805(R)), 2009.

[35] A. Imamoglu M. Fleischhauer and J.P. Marangos. Electromagnetically induced transparency: Optics in coherent media. Rev. Mod. Phys., 77(2), 2005.

[36] A. Lipsich A. Lezama, S. Barreiro and A.M. Akulshin. Coherent two-field spectroscopy of degenerate two-level systems. Phys. Rev. A., 61(013801), 1999. 
[37] P.Valente A. Lipsich, S. Barreiro and A. Lezama. Electromagnetically induced absorption in magneto-optically trapped atoms. arXiv:physics/0009091v1 [physics.atom-ph], 2008.

[38] Marlan O. Scully and M. Suhail Zubairy. Quantum Optics. Cambridge University Press, 2nd edition, 2001.

[39] Dieter Suter. The Physics of Laser-Atom interactions. Cambridge University Press, 1nd edition, 1997.

[40] Alain Aspect Gilbert Grynberg and Claude Fabre. Introduction to Quantum Optics. Cambridge University Press, 1st edition, 2010.

[41] T. Yabuzaki et al. New type of high-resolution spectroscopy with a diode laser. Phys. Rev. Lett., 67(2453), 1991.

[42] C.W.Gardiner. Handbook of Stochastic Methods. Springer-Verlag, 1990.

[43] Claude Cohen-Tannoudji Jacques Dupont-Roc and Gilbert Grynberg. AtomPhoton Interactions - Basic Processes and Applications. John Wiley \& Sons, 1992.

[44] Daniel Adam Steck. Rubidium 85 d line data. University of Oregon, 2008.

[45] Cristopher J. Foot. Atomic Physics. Oxford University Press, 1st edition, 2005.

[46] P.D. Lett et al. Optical molasses. J. Opt Soc. Am. B, 6(11), 1989.

[47] Y. Castin et al. Limit of doppler cooling. J. Opt Soc. Am. B, 6(11), 1989.

[48] H.J. Metcalf and P. Straten. Laser Cooling and Trapping. Springer-Verlag, 1st edition, 1999.

[49] D. Budker et al. Resonant nonlinear magneto-optical effects in atoms. Rev. Mod. Phys., 74(4), 2002.

[50] Marcelo Martinelli. Compressão de ruído Quântico e efeitos transversos em Osciladores Paramétricos Óticos. PhD thesis, Universidade de São Paulo, Fevereiro 2002 .

[51] L.Ricci et al. Ellectromagnetically induced transparency: Optics in coherent media. Opt. Commun, 177(4), 1995.

[52] C.J. Hawlthorn;K.P. Weber and R.E. Scholten. Littrow configuration tunable external cavity diode laser with fixed direction output beam. Rev. Sci. Instrum., $72(12), 2001$. 
[53] Warren Nagourney. Quantum Lectronics for Atomic Physics. Oxford University Press, 1st edition, 2010.

[54] E.A. Donley et al. Double-pass acousto-optic modulator system. Rev. Sci. Instrum., 76(063112), 2005.

[55] F. B. M. dos Santos and J. W. R. Tabosa. Application of electromagnetically induced transparency for cold-atom velocimetry. Phys. Rev. A., 73(023422), 2006.

[56] Rafael Alves de Oliveira. Correlação de intensidade em transparência electromagnéticamente induzida. PhD thesis, Universidade Federal de Pernambuco, Previsto 2013-14. 